\title{
The Error Propagation Analysis of the Received Signal Strength-based Simultaneous Localization and Tracking in Wireless Sensor Networks
}

\author{
Bingpeng Zhou ${ }^{\dagger}$, Qingchun Chen ${ }^{\dagger}$, Pei Xiao ${ }^{\ddagger}$ \\ ${ }^{\dagger}$ Southwest Jiaotong University, Chengdu, Sichuan 610031, China \\ ${ }^{\ddagger}$ University of Surrey, Guildford, Surrey GU2 7XH, UK \\ zhoubingpeng@163.com, qcchen@swjtu.edu.cn, p.xiao@surrey.ac.uk
}

\begin{abstract}
Simultaneous localization and tracking (SLAT) in wireless sensor networks (WSNs) involves tracking the mobile target while calibrating the nearby sensor node locations. In practice, a localization error propagation (EP) phenomenon will arise, due to the existence of the latest tracking error, target mobility, measurement error and reference node location errors. In this case, the SLAT performance limits are crucial for the SLAT algorithm design and WSN deployment, and the study of localization EP principle is desirable. In this paper, we focus on the EP issues for the received signal strength-based SLAT scheme, where the measurement accuracy is assumed to be spatialtemporal-domain doubly random due to the target mobility, environment dynamics and different surroundings at different reference nodes. Firstly, the Cramer-Rao lower bound (CRLB) is derived to unveil both the target tracking EP and the node location calibration EP. In both cases, the EP principles turn out to be in a consistent form of the Ohm's Law in circuit theory. Secondly, the asymptotic CRLB analysis is then presented to reveal that both EP principles scale with the inverse of sensor node density. Meanwhile, it is shown that, the tracking and calibration accuracy only depends on the expectation of the measurement precision. Thirdly, the convergence conditions, convergence properties and the balance state of the target tracking EP and the location calibration EP are examined to shed light on the EP characteristics of the SLAT scheme. Finally, numerical simulations are presented to corroborate the EP analysis.
\end{abstract}

Index Terms-Error propagation, random measurement accuracy, simultaneous localization and tracking, WSNs.

\section{INTRODUCTION}

$\mathbf{S}$ IMULTANEOUS localization and tracking (SLAT) of a mobile target has attracted tremendous interests with the rapid advances in wireless sensor networks (WSNs) [1], [2], e.g., the location-based services [3], warehousing management, location-aware security [4], [5], location-based network routing [6], [7], and shopping mall navigation [8].

The SLAT problem is to track the mobile target location (referred to as "tracking" hereafter) while calibrating the locations of network nodes around (referred to as "calibration" hereafter). In principle, the mobile target tracking can be considered as the localization cooperation in the temporaldomain [9], while the sensor node location (and the cooperative network localization [10] as well) can be regarded as

This work was supported by the NSFC (No. 61271246). This work was partly supported by the Royal Academy of Engineering Award (batch reference 1314-2). the localization cooperation in the spatial-domain [11]. It is highly desirable to study the performance limits of the SLAT scheme and its error propagation (EP) behaviours for both algorithm design and wireless sensor network deployment. The EP phenomenon arises from the uncertainties $(e . g$. , the previous mobile tracking error, target mobility, measurement error and reference node location errors) propagating within the target tracking and the sensor node location calibration in the SLAT process.

In this paper, the EP issue is studied for the SLAT scheme in wireless sensor networks based on the received signal strength (RSS) measurements, due to its compatibility to the communication infrastructure [12]. In fact, the RSS measurement is a promising choice in closed indoor environments (e.g., shopping mall or underground parking) where the global positioning system (GPS) signal is unavailable [13]. However, there are still several theoretical challenges, for instances, (1) the error propagation principle of mobile tracking \& node location calibration and (11) the asymptotic performance limits over dependent factors, such as reference node density, reference node location error and measurement accuracy. In this paper, we focus on the following specific challenges.

Firstly, the error propagation principle of the RSS-based SLAT scheme in a WSN is not yet completely known in theory to date, which should be studied to gain insights into the inherent mechanism of temporal-spatial-domain localization cooperation in the SLAT scheme, and to examine the dominate factors affecting localization performance. In addition, the conditions that guarantee the tracking \& calibration error propagation convergence are also important for the practical SLAT scheme development [14] and network design.

Secondly, the measurement accuracies at different sensor nodes might be different from each other ("spatial-domain randomness") due to their different levels of shadowing, device orientations, strength of thermal noises and surrounding environments [15]. On the other hand, the measurement accuracies possibly change over time ("temporal-domain randomness") due to target mobility, ${ }^{1}$ wherein the reference node set will change at the same time. In addition, in a dynamic environ-

\footnotetext{
${ }^{1}$ Even in a low-mobility scenario, the measurement accuracies may still change over a long time scale.
} 
ment (e.g., a shopping mall crowded with moving people), the measurement accuracy is time varying in nature. The impact of the spatial-temporal-domain doubly random measurement accuracy on mobile tracking performance and error propagation behaviour need be analyzed to gain insights into its inherent philosophy.

Thirdly, the sensor node locations are inaccurate in practical applications [16] due to the inevitable errors in their initial location acquisitions. The sensor node location error is one of the main sources to account for localization error propagation. In such a case, the reference node location uncertainties should be considered in the SLAT scheme to examine its impact on the tracking \& calibration accuracy and its performance limit. The analytical results can provide guidelines for the SLAT algorithm design to reap the full potential gains, when the statistical knowledge of reference node location errors is available in mobile tracking \& calibration [14]

Finally, the reference node number is another crucial factor that affects the achievable SLAT performance [17]. Particularly in an energy-constrained wireless network [18], [19], [20], the tradeoff between tracking \& calibration accuracy and energy consumption should be considered. Hence, it is highly desirable to quantitatively study the scaling rule of tracking accuracy and energy consumption versus the reference node number, thus providing guidelines for the SLAT algorithm design to strike a balance between SLAT performance and implementation cost. In fact, the node selection strategy in localization and tracking scheme was addressed in [21] to highlight the impact of reference node set size and the resultant energy efficiency.

Considering the aforementioned challenges, in this paper we strive to answer the following two fundamental questions:

- How do the spatial-temporal randomness of measurement accuracy, sensor node density and the sensor node location errors affect the SLAT performance?

- How do the tracking error and the calibration error of the SLAT scheme propagate over time?

Considering the measurement accuracies at reference nodes are random and nondeterministic in the spatial-temporal domain, in this paper, we introduce an independent and identical Wishart density, which is the conjugate priori of the precision of a Gaussian variable [22], [23], to characterize the statistical dynamics and randomness of measurement accuracies at reference nodes. In addition, the Gaussian density is employed to characterize the initial location errors of sensor nodes [24], [25]. The associated Cramer-Rao lower bound (CRLB) is utilized to disclose both the mobile target tracking EP and the sensor node location calibration EP. Interestingly, it is shown that, both EP principles resemble the Ohm's Law in circuit theory, where the reference node location error, measurement error, the latest tracking error and target mobility behave like the resistances connected in parallel or in series, all of which contribute the tracking and calibration performance. In addition, it is disclosed that, the essence of localization cooperation in the temporal-domain (e.g., mobility tracking) and the spatial-domain (i.e., node location calibration) lies in the related localization information propagation. Moreover, the error propagation properties and the convergence conditions are also studied to show under what conditions the error propagation converges. It is revealed that, the mobile tracking precision increases over time when the measurement information could compensate for the information loss in the location prediction. Moreover, the information loss increases with the increasing of the latest tracking precision. Consequently, the tracking precision will converge to a balance state till the information loss and the measurement information are wellmatched in strength. Furthermore, the asymptotic CRLB analysis is presented to quantify the impact of the nondeterministic measurement accuracies, sensor node density and reference node location errors on the SLAT accuracy. It is shown that, both tracking and calibration accuracies scale linearly with the sensor density (or the number of reference nodes within a fixed sensing range).

The main contributions of this paper are three-fold.

- The EP principles of mobile target tracking and sensor location calibration in the SLAT issue are revealed, which turn out to resemble the Ohm's Law in circuit theory. The obtained EP principles can be readily extended to linear Gaussian and nonlinear non-Gaussian filtering problems.

- The convergence conditions and the properties of tracking and calibration EP behaviors are studied to shed lights on the localization information exchange for the mobile target prediction, tracking and the reference node location calibration.

- The asymptotic performance limits are derived to reveal the impact of those dependent factors like reference node density, reference node location errors, target transition model and measurement accuracy on the SLAT performance, which is important for practical algorithm development and network design.

The remainder of this paper is organized as follows. Section II introduces the related prior work. Section III presents the system model. Section IV summarizes the corresponding statistical model. Error propagation for both target tracking and sensor node location calibration is studied in Section V. Section VI provides a useful and interesting physical interpretation of the associated EP philosophy. In Section VII, the EP convergence conditions and properties are analysed. In Section VIII, the asymptotic CRLB analysis is conducted. Simulations results are presented in Section IX to corroborate the EP laws and the asymptotic analysis. Finally, conclusions are drawn in Section X. The mathematical symbol notations used in this paper are summarized in Table I.

\section{RELATED WORK}

Given an unbiased estimator, the CRLB is usually used as a lower bound on the variance of estimation error [26], which can indicate the performance limit of an estimator.

In addition to $\mathrm{CRLB},{ }^{2}$ there are also other metrics that can serve as the estimation error lower bound, e.g., Barankin

\footnotetext{
${ }^{2}$ Even in the framework of CRLB, there are numerous versions dependent on the specific situations, such as non-Bayesian CRLB when only measurement information is accessible [26], [27], Bayesian CRLB when the priori information of random variable is available [28], and hybrid CRLB when there are multiple random variables [29], [30]
} 
TABLE I

OPERATION SYMBOL NOTATIONS

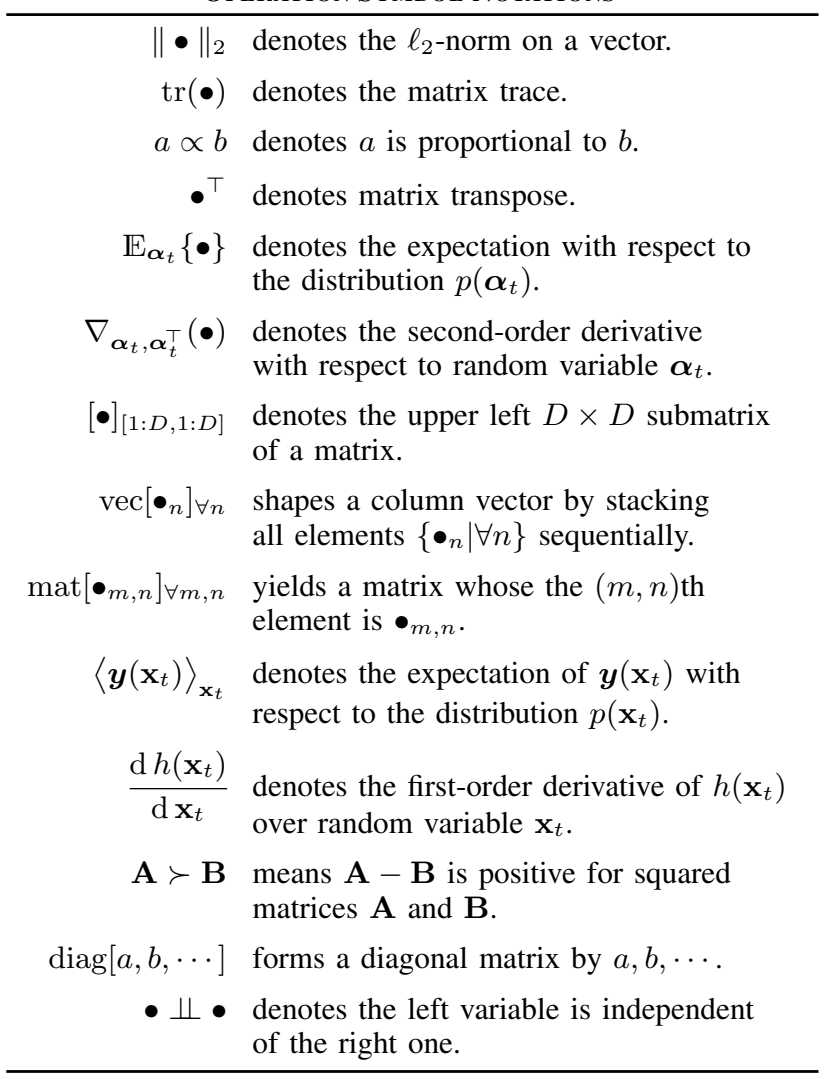

bound [31], [32], [33], Bobrovsky bound [34], Ziv-Zakai bound [35], [36], and Weiss-Weinstein bound [37]. Compared with CRLB, these bounds are more compact when the asymptotic condition (i.e., the measurement sample size is sufficiently large) does not hold. The use of these bounds is dependent on whether the unknown parameters are random (or nonrandom) [32], [36], [37]. In this paper, we focus on the CRLB to study the SLAT performance and the EP mechanism, due to its wide applicability and succinct expression.

There are a number of previous research efforts devoted to identifying wireless localization/tracking performance limits in different environments with different measurement modalities, by means of the CRLB analysis. Generally, in different localization scenarios with different measurement options, such as RSS, time-of-arrival, angle-of-arrival and time-difference-of-arrival, there are different error sources. It is shown in [38] that the error sources of time-based localization (such as time-of-arrival and time-difference-ofarrival) include multipath, excess delay and blockage. The associated performance limit was also analyzed in [38] to demonstrate the effect of multipath, interference and bandwidth. Among these error sources, the multipath or non-lineof-sight propagation is particularly detrimental for the timebased localization performance [39]. The fundamental limits of cooperative and noncooperative localization were studied in [40] and [41] to reveal the impact of multipath and nonline-of-sight transmission in broadband wireless networks. For the RSS-based localization and tracking, the associated CRLB analysis was presented in [42] to benchmark the tracking error in WSNs. The CRLB analysis was also conducted in [43] to quantify the effects of reference errors on the localization performance. In contrast to the lower bound, an upper bound was derived in [44] for different types of measurement errors. In [45], the concept of information coupling was proposed for cooperative network localization. The fundamental limits were studied in [46] to explicate the temporal-domain propagation of mobile tracking errors, where different types of wireless network measurements and performance requirements in various scenarios are considered. The navigation information evolution was studied in [9] to highlight the spatial and temporal-domain localization cooperation in navigation networks.

The theoretical performance analysis can provide guidelines for localization and tracking system design, such as reference node selection and network deployment. For instance, the CRLB can be used as a performance metric in the power allocation design of practical localization applications to achieve the optimal localization accuracy, as shown in [47]. In [48], an optimal sensor node placement scheme was proposed to minimize localization error. Similarly, a CRLBdriven sensor node selection strategy and placement scheme were investigated in [49] to minimize the localization error for mobile target. In addition, it is proposed in [50] to select the best sensor node measurement for the optimal update of target location estimation. The optimal sensor node selection scheme was studied in [51], [52] and [53] for target localization and mobile tracking, where either the entropy or information of the posterior target location distribution was employed. There are also other reference sensor node selection schemes proposed in [54], [55] and [56] to achieve a reasonable tradeoff between the localization performance and energy efficiency.

Moreover, there are a multitude of earlier research efforts in designing practical localization and tracking schemes in various scenarios with different considerations. For example, in order to reduce the number of anchor nodes, a distributed sensor localization method was proposed in [57]. Subsequently, a modified version of this distributed localization method was proposed in [58] to improve the robustness by further suppressing the noisy distance measurements and by harnessing the dynamic communication links. A distributed variational filtering-based approach was proposed in [42] to derive the efficient RSS-based SLAT scheme in WSNs. Furthermore, in [38], the time-of-arrival-based localization techniques were reviewed, and different strategies of the first signal path detection demonstrated. In order to mitigate the effect of RSS measurement variation and instability, a real-time path loss model training-based indoor localization was proposed in [59]. In such a case, the burdensome calibration and radio-map information are no longer required. A comprehensive survey was presented in [60] to introduce the existing measurement methods in narrowband, wideband and ultraband systems, as well as the algorithm design issue associated with the propagation model and pattern recognition-based methods for indoor localization.

Furthermore, the practical experiments have been conducted to demonstrate the achievable localization/tracking performance against various disturbance sources or dependent factors. In [61], the network experiments were carried out 
to examine the performance of cooperative network localization with range and waveform measurements, which provides insight into the potential value of cooperative techniques and environmental information. A realistic indoor localization experiment was performed in [62] to examine the off-line RSS propagation model tuning and different localization strategies. In order to characterize the measurement diversity (i.e., the combination of multiple measurement methods) on the navigation system performance, an extensive experiment campaign was performed in [63]. In addition, the particle filteringbased navigation algorithm was employed in the experiment to quantify the benefits of diverse data fusion [63].

Recently, a general Inertial navigation framework was proposed in [64] to exploit contextual information, which improves navigation performance. In applications, the sampling and reconstruction theory of a finite-energy signal with uncertainties in [65] can be utilized to devise practical mobile target tracking algorithms with reference node location errors. Moreover, a power management scheme for cooperative localization was studied in [66], where a game-theoretical method was employed.

All these research efforts provide valuable references for the wireless localization and tracking design in terms of system optimization [47], [48], [49], [51], [52], [53], algorithms development [42], [57], [58], [59], [60], performance limits [38], [40], [41], [43], [44], [45], [46] and environmental experiments [61], [62], [63], with various measurement choices and practical constraints (e.g., indoor [67], outdoor [68], mobile tracking [42], non-line-of-sight [39], [40], [41] and limited energy budget [18], [19], [20]). However, there is no relevant work on the investigation of the EP philosophy for the SLAT scheme in WSNs, particularly in environments with the spatial-temporal-domain random measurement accuracy. In addition, the study of EP properties, their relationship with sensor density [17], and the conditions under which the EP converges are needed for the practical SLAT scheme design, and this is exactly the motivation for the work presented in this paper.

\section{SySTEM MODEL}

\section{A. Network Model}

The WSN under study is depicted in Fig. 1, where all sensor nodes are assumed to be randomly and uniformly distributed inside a deployment area. Due to the inevitable errors in the initial location acquisitions of sensor nodes, we assume that all sensor node locations are inaccurate. Let $s^{i}$ denote the true (but unknown) location of the $i$ th sensor node, $i=1: M$, where $M$ denotes the total number of sensor nodes inside the tracking area. A mobile target moves inside this area, whose location at time instant $t$ is denoted by a $D$-dimensional vector $\mathbf{x}_{t}$, which is unknown and to be tracked.

Assume the sensing range of each sensor node is $r_{s}$ metres. All the sensor nodes within the sensing range around the target form a temporary reference set to help track the target. Let's define the index set associated with these reference nodes as

$$
\Psi_{t}=\left\{i:\left\|s^{i}-\mathbf{x}_{t}\right\|_{2} \leq r_{s}, \forall i=1, \cdots, M\right\}
$$

where $\|\bullet\|_{2}$ stands for the $\ell_{2}$-norm on a vector. Assume the size of this reference cluster $\Psi_{t}$ is $M_{t}$.

Let the (unknown) true location of the $i$ th reference node in $\Psi_{t}$ be $\mathbf{s}_{t}^{i}$, while its coarse location is denoted by $\boldsymbol{\mu}_{t}^{i}$. The coarse location is assumed to be inaccurate with precision matrix $\mathbf{U}_{t}^{i}$. In general, the (unknown) true location $\mathbf{s}_{t}^{i}$ is assumed to be a Gaussian distributed variable with the mean vector $\boldsymbol{\mu}_{t}^{i}$ and the precision matrix $\mathbf{U}_{t}^{i}$, namely, [24], [25]

$$
\mathbf{s}_{t}^{i} \sim \mathcal{N}\left(\mathbf{s}_{t}^{i} \mid \boldsymbol{\mu}_{t}^{i}, \mathbf{U}_{t}^{i}\right), \forall i=1: M_{t},
$$

where independent sensor location precisions $\mathbf{U}_{t}^{i}$ is assumed. The sensor location uncertainty is defined as the inverse of its precision. The reference variable vector is defined as as $\mathbf{s}_{t}=\left[\mathbf{s}_{t}^{1} ; \ldots ; \mathbf{s}_{t}^{M_{t}}\right]$.

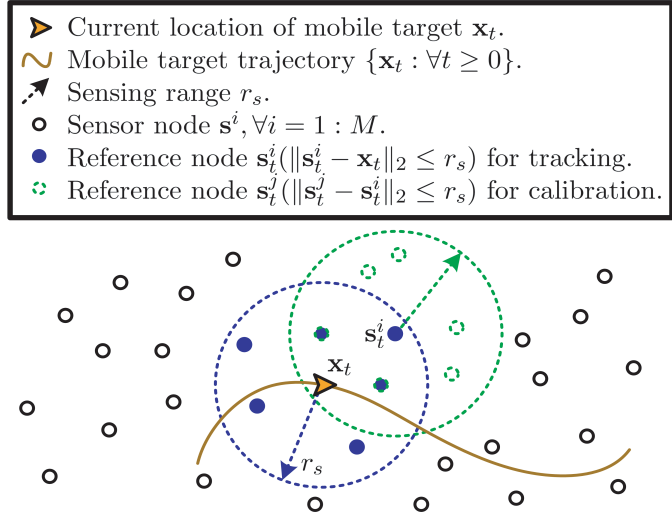

Fig. 1. Illustration of the sensor network deployment.

Once completing the target tracking, the SLAT scheme starts to calibrate those reference senor nodes with the assistance of the localized target and other sensor nodes nearby. ${ }^{3}$ For the location calibration of the objective node $\mathbf{s}_{t}^{i}$, we assume that, there are $N_{t} \geq 0$ nearby sensor nodes available as reference nodes in addition to the mobile target. Let the index set of reference cluster formed by these sensor nodes be denoted as

$$
\Phi_{t}^{i}=\left\{j:\left\|s^{j}-\mathbf{s}_{t}^{i}\right\|_{2} \leq r_{s}, \forall s^{j} \neq \mathbf{s}_{t}^{i}\right\} .
$$

Let's define a vector associated with reference sensor nodes of the objective node $\mathbf{s}_{t}^{i}$ as $\mathbf{c}_{t}^{i}=\left[\mathbf{s}_{t}^{1} ; \cdots ; \mathbf{s}_{t}^{N_{t}}\right]$.

In addition, when $N_{t}=0$, the SLAT problem under study is simplified to the traditional case where only the localized target helps calibrate the objective node, i.e., there is no localization cooperation among sensor nodes [42].

\section{B. Measurement Model}

In this paper, the RSS measurement in shadow fading environment is assumed for the SLAT issue, where each sensor node that has perceived the mobile target will reply the target with the associated RSS measurements. The RSS measurement (in $\mathrm{dB}$ ) associated with reference sensor node $\mathbf{s}_{t}^{i}$ and the target $\mathbf{x}_{t}$ is modeled as

$$
\mathrm{z}_{t}^{i}=h\left(\mathbf{x}_{t}, \mathbf{s}_{t}^{i}\right)+\epsilon_{t}^{i}, \forall i \in \Psi_{t}
$$

${ }^{3}$ Hereafter, when we refer to reference node location calibration, we call the reference node that is being calibrated as 'the objective node' to distinguish it from other reference nodes. 
where $\epsilon_{t}^{i}$ denotes the measurement error (for instance, due to the shadowing effect), and $h\left(\mathbf{x}_{t}, \mathbf{s}_{t}^{i}\right)$ is the measurement function dependent on the distance between the target and the sensor node $\mathbf{s}_{t}^{i}$, which is specified as [42], [69]

$$
h\left(\mathbf{x}_{t}, \mathbf{s}_{t}^{i}\right)=P_{T}-P_{0}-10 \gamma \log _{10} \frac{\left\|\mathbf{s}_{t}^{i}-\mathbf{x}_{t}\right\|_{2}}{d_{0}},
$$

where $P_{T}$ is the transmit power, $P_{0}$ denotes the power loss associated with the distance $d_{0}$, and $\gamma$ denotes the path loss exponent (in general $\gamma \in[2,4][70]$ ). The measurement function describes the associated path loss component. Furthermore, by defining a constant $\phi=P_{T}-P_{0}+10 \gamma \log _{10} d_{0}$ that is independent to the distance $\left\|\mathbf{s}_{t}^{i}-\mathbf{x}_{t}\right\|_{2}$, the RSS measurement function $h\left(\mathbf{x}_{t}, \mathbf{s}_{t}^{i}\right)$ can be equivalently expressed in a compact form as

$$
h\left(\mathbf{x}_{t}, \mathbf{s}_{t}^{i}\right)=\phi-10 \gamma \log _{10}\left\|\mathbf{s}_{t}^{i}-\mathbf{x}_{t}\right\|_{2} .
$$

In the same way, the RSS measurement between the objective sensor node $\mathbf{s}_{t}^{i}$ and its reference node $\mathbf{s}_{t}^{j}$ is given by

$$
y_{t}^{i, j}=h\left(\mathbf{s}_{t}^{i}, \mathbf{s}_{t}^{j}\right)+\epsilon_{t}^{i, j}, \forall j \in \Phi_{t}^{i},
$$

where the associated RSS measurement function $h\left(\mathbf{s}_{t}^{i}, \mathbf{s}_{t}^{j}\right)$ is specified as

$$
h\left(\mathbf{s}_{t}^{i}, \mathbf{s}_{t}^{j}\right)=\phi-10 \gamma \log _{10}\left\|\mathbf{s}_{t}^{i}-\mathbf{s}_{t}^{j}\right\|_{2} .
$$

Although we focus on the RSS-based measurement in this paper, it should be noted that the following analysis can be readily generalized to any form of measurement function.

In addition, the measurement noises $\epsilon_{t}^{i}$ and $\epsilon_{t}^{i, j}$ in Eqs. (4) and (7) are assumed to be independently Gaussian distributed with zero-mean and precision scalar $\mathrm{w}_{t}^{i}$ and $\mathrm{w}_{t}^{i, j}$, respectively, namely, $\epsilon_{t}^{i} \sim \mathcal{N}\left(\epsilon_{t}^{i} \mid 0, \mathrm{w}_{t}^{i}\right)$ and $\epsilon_{t}^{i, j} \sim \mathcal{N}\left(\epsilon_{t}^{i, j} \mid 0, \mathrm{w}_{t}^{i, j}\right) .{ }^{4}$

In practical applications, at each time instant, the measurement precision at differen reference node might be different from each other (i.e., spatial-domain randomness), due to different shadowing fading levels, different device orientations, different thermal noise levels and different surrounding obstructions [15]. The measurement precision is also timevarying (i.e., temporal-domain randomness), due to the target mobility, especially when the reference node set is changed during the target tracking. In addition, in a dynamic environment (such as shopping mall crowded with moving people), the measurement accuracies become time varying. Hence, their real-time true values are difficult to obtain.

In order to capture the temporal-domain random measurement precision of different sensor nodes at different time instant, we introduce a Wishart hyperpriori density (which is the conjugate priori of the precision of a Gaussian random variable [22], [23]) to model each measurement precision $\mathrm{w}_{t}^{i}$ and $\mathrm{w}_{t}^{i, j}$, namely,

$$
\begin{aligned}
\mathrm{w}_{t}^{i} & \sim \mathcal{W}\left(\mathrm{w}_{t}^{i} \mid \Omega, \psi\right), \forall i \in \Psi_{t}, \forall t=1: K, \\
\mathrm{w}_{t}^{i, j} & \sim \mathcal{W}\left(\mathrm{w}_{t}^{i, j} \mid \Omega, \psi\right), \forall j \in \Phi_{t},
\end{aligned}
$$

\footnotetext{
${ }^{4}$ In fact, in Eqs. (4) and (7), the measurement functions $h\left(\mathbf{x}_{t}, \mathbf{s}_{t}^{i}\right)$ and $h\left(\mathbf{s}_{t}^{i}, \mathbf{s}_{t}^{j}\right)$ stand for the large-scale fading contribution to the RSS measurement at the mobile target $\mathbf{x}_{t}$ and the objective sensor node $\mathbf{s}_{t}^{i}$, respectively, while $\epsilon_{t}^{i, j}$ and $\epsilon_{t}^{i}$ correspond to the shadow fading effects.
}

where the positive scalar $\Omega$ denotes its prior scale parameter and $\psi$ denotes the associated degree of freedom (DoF). In this paper, an independent and identical distribution for $\mathrm{w}_{t}^{i}$ and $\mathrm{w}_{t}^{i, j}$ with the same parameters $\Omega$ and $\psi$ is assumed for measurement noises, since the corresponding measurement data $\mathrm{z}_{t}^{i}$ and $y_{t}^{i, j}$ are obtained inside an identical tracking \& calibration area.

Noted that the spatial-temporal-domain random measurement model considered in this paper can subsume the special case when the measurement precision is deterministic and known, in which case $\psi \Omega^{2} \rightarrow 0$. Let $\mathbf{w}_{t}=\left[\mathrm{w}_{t}^{1}, \cdots, \mathrm{w}_{t}^{M_{t}}\right]^{\top}$ denote the precision vector for target tracking, and let $\boldsymbol{\omega}_{t}^{i}=$ $\left[\mathrm{w}_{t}^{i, 1}, \cdots, \mathrm{w}_{t}^{i, N_{t}}\right]^{\top}$ denote the measurement precision vector for node location calibration.

For continence, we define an $M_{t}$-dimensional measurement vector as $\mathbf{z}_{t}=\left[\mathrm{z}_{t}^{1} ; \cdots ; \mathrm{z}_{t}^{M_{t}}\right]$, which is stacked by the measurements between the mobile target and reference sensor nodes. Similarly, let $\boldsymbol{y}_{t}=\left[\mathrm{z}_{t}^{i}, y_{t}^{i, 1} ; \cdots ; y_{t}^{i, N_{t}}\right]$ stand for the measurement vector for the objective node $\mathbf{s}_{t}^{i .5}$.

\section{Target Movement}

In this paper we consider a general random walking model, where the current target position $\mathbf{x}_{t}$ is assumed to move from its previous location according to the following model

$$
\mathbf{x}_{t}=\mathbf{x}_{t-1}+\boldsymbol{\varsigma}_{t},
$$

where $\varsigma_{t}$ denotes the location transition vector. Generally, we assume $\boldsymbol{s}_{t}$ to be a Gaussian variable with zero-mean and the precision matrix $\boldsymbol{\chi}$, i.e., $\boldsymbol{\varsigma}_{t} \sim \mathcal{N}\left(\boldsymbol{\varsigma}_{t} \mid \mathbf{0}, \boldsymbol{\chi}\right)$ [42], [71].

An extended Gaussian model is employed in [72], [73], [74] to characterize those underlying statistics information in the target mobility. To facilitate the EP analysis of SLAT, we only consider the naive but general mobility model in Eq. (11).

\section{Problem Statement}

The SLAT scheme is first to track the mobile target location $\mathbf{x}_{t}$, based on the coarse locations and precisions $\left\{\boldsymbol{\mu}_{t}^{i}, \mathbf{U}_{t}^{i}: \forall i \in\right.$ $\left.\Psi_{t}\right\}$ of reference nodes as well as the current RSS observation $\left\{\mathrm{z}_{t}^{i}: \forall i \in \Psi_{t}\right\}$, and then to calibrate each inaccurate reference node $\mathbf{s}_{t}^{i}$ based on the measurement data $\left\{y_{t}^{i, j}: \forall j \in \Phi_{t}^{i}\right\}$ from other reference nodes $\left\{\boldsymbol{\mu}_{t}^{j}, \mathbf{U}_{t}^{j}: \forall j \in \Phi_{t}^{i}\right\}$ nearby and $\mathrm{z}_{t}^{i}$ associated with the mobile target $\mathbf{x}_{t}$.

In this paper, we investigate the error propagation principles of the above SLAT scheme. In particular, we aim at finding answers to the following fundamental questions:

- How do the spatial-temporal random measurement accuracy, sensor node density and the sensor node location errors affect the SLAT performance?

- How do the tracking error and the calibration error of the SLAT scheme propagate over time?

\section{Statistical Model}

Prior to studying the EP principles of the SLAT scheme, we introduce the statistical models associated with mobile tracking and node location calibration, which will be used in the subsequent EP analysis.

\footnotetext{
${ }^{5}$ We drop the superscript $i$ in the measurement vector $\boldsymbol{y}_{t}$ of the objective node $\mathbf{s}_{t}^{i}$ whenever no ambiguity arises, for the sake of simplicity.
} 


\section{A. Tracking Knowledge}

Let's define a $\left(D+M_{t} D+M_{t}\right)$-dimensional complete state variable associated with target tracking as $\boldsymbol{\alpha}_{t}=\left[\mathbf{x}_{t} ; \mathbf{s}_{t} ; \mathbf{w}_{t}\right]$.

We assume that, the current measurements $\left\{\mathrm{z}_{t}^{i} \mid \forall i \in \Psi_{t}\right\}$ conditioned on $\boldsymbol{\alpha}_{t}$ are mutually independent, so that the corresponding joint likelihood function is given by

$$
p\left(\mathbf{z}_{t} \mid \boldsymbol{\alpha}_{t}\right)=\prod_{i \in \Psi_{t}} \frac{\left|\mathrm{w}_{t}^{i}\right|^{\frac{1}{2}}}{\sqrt{2 \pi}} \exp \left(-\frac{1}{2} \mathrm{w}_{t}^{i}\left(\mathrm{z}_{t}^{i}-h\left(\mathbf{x}_{t}, \mathbf{s}_{t}^{i}\right)\right)^{2}\right) .
$$

Considering the complete state variable $\boldsymbol{\alpha}_{t}$, the associated state transition distribution can be formed as

$$
p\left(\boldsymbol{\alpha}_{t} \mid \boldsymbol{\alpha}_{t-1}\right)=p\left(\mathbf{x}_{t} \mid \mathbf{x}_{t-1}\right) \prod_{i \in \Psi_{t}} \mathcal{N}\left(\mathbf{s}_{t}^{i} \mid \boldsymbol{\mu}_{t}^{i}, \mathbf{U}_{t}^{i}\right) p\left(\mathrm{w}_{t}^{i}\right),
$$

where the measurement precision's priori distribution is defined as $p\left(\mathrm{w}_{t}^{i}\right)=\mathcal{W}\left(\mathrm{w}_{t}^{i} \mid \Omega, \psi\right)$ based on Eq. (9), and the location transition distribution of mobile target is cast as

$$
p\left(\mathbf{x}_{t} \mid \mathbf{x}_{t-1}\right)=\mathcal{N}\left(\mathbf{x}_{t} \mid \mathbf{x}_{t-1}, \boldsymbol{\chi}\right),
$$

according to the target movement model in Eq. (11).

One can see from Eq. (13) that, only variable $\mathbf{x}_{t}$ relates to its previous state $\mathbf{x}_{t-1}$ and thus only the state variable $\mathbf{x}_{t-1}$ can provide its history information for the prediction of the current complete state $\boldsymbol{\alpha}_{t}$.

Given the posterior distribution $p\left(\mathbf{x}_{t-1} \mid \mathbf{z}_{1: t-1}\right)$ with respect to the target location, based on the location transition distribution in Eq. (14), the location prediction distribution can thus be calculated as

$$
p\left(\mathbf{x}_{t} \mid \mathbf{z}_{1: t-1}\right)=\int p\left(\mathbf{x}_{t} \mid \mathbf{x}_{t-1}\right) p\left(\mathbf{x}_{t-1} \mid \mathbf{z}_{1: t-1}\right) \mathrm{d} \mathbf{x}_{t-1} .
$$

Let $\boldsymbol{J}_{\mathrm{BE}}^{\mathbf{x}_{t-1}}$ denote the Bayesian estimation (BE)-based Fisher information matrix (FIM) for the latest target tracking. According to Bayesian statistics, the estimation errors asymptotically follow the Gaussian distribution as the observation sample size approaches infinity [26]. For the sake of generality, we assume the previous posteriori distribution can be asymptotically approximated by a Gaussian distribution with precision matrix $\boldsymbol{J}_{\mathrm{BE}}^{\mathbf{x}_{t-1}}$, namely,

$$
p\left(\mathbf{x}_{t-1} \mid \mathbf{z}_{1: t-1}\right) \approx \mathcal{N}\left(\mathbf{x}_{t-1} \mid \mathbf{x}_{t-1}^{\sharp}, \boldsymbol{J}_{\mathrm{BE}}^{\mathbf{x}_{t-1}}\right),
$$

where $\mathbf{x}_{t-1}^{\sharp}$ is the latest posteriori estimation of target location. Hence, the prediction distribution is further expressed as

$$
\begin{aligned}
p\left(\mathbf{x}_{t} \mid \mathbf{z}_{1: t-1}\right) & \approx \int \mathcal{N}\left(\mathbf{x}_{t} \mid \mathbf{x}_{t-1}, \boldsymbol{\chi}\right) \mathcal{N}\left(\mathbf{x}_{t-1} \mid \mathbf{x}_{t-1}^{\sharp}, \boldsymbol{J}_{\mathrm{BE}}^{\mathbf{x}_{t-1}}\right) \mathrm{d} \mathbf{x}_{t-1} \\
& =\mathcal{N}\left(\mathbf{x}_{t} \mid \mathbf{x}_{t-1}^{\sharp}, \boldsymbol{\Theta}_{t}^{p}\right),
\end{aligned}
$$

where the current prediction precision $\Theta_{t}^{p}$ is derived according to the Gaussian distribution properties as

$$
\boldsymbol{\Theta}_{t}^{p}=\left(\left(\boldsymbol{J}_{\mathrm{BE}}^{\mathbf{x}_{t-1}}\right)^{-1}+\chi^{-1}\right)^{-1},
$$

Combining Eqs. (14) and (17), the prediction distribution of complete state variable $\boldsymbol{\alpha}_{t}$ is derived as

$$
\begin{aligned}
& p\left(\boldsymbol{\alpha}_{t} \mid \mathbf{z}_{1: t-1}\right)=\int p\left(\boldsymbol{\alpha}_{t-1} \mid \mathbf{z}_{1: t-1}\right) p\left(\boldsymbol{\alpha}_{t} \mid \boldsymbol{\alpha}_{t-1}\right) \mathrm{d} \boldsymbol{\alpha}_{t-1} \\
= & \int p\left(\mathbf{x}_{t-1} \mid \mathbf{z}_{1: t-1}\right) p\left(\mathbf{x}_{t} \mid \mathbf{x}_{t-1}\right) \mathrm{d} \mathbf{x}_{t-1} \prod_{i \in \Psi_{t}} p\left(\mathbf{s}_{t}^{i}\right) p\left(\mathrm{w}_{t}^{i}\right) \\
\approx & \mathcal{N}\left(\mathbf{x}_{t} \mid \mathbf{x}_{t-1}^{\sharp}, \boldsymbol{\Theta}_{t}^{p}\right) \prod_{i \in \Psi_{t}} \mathcal{N}\left(\mathbf{s}_{t}^{i} \mid \boldsymbol{\mu}_{t}^{i}, \mathbf{U}_{t}^{i}\right) \mathcal{W}\left(\mathrm{w}_{t}^{i} \mid \Omega, \psi\right) .
\end{aligned}
$$

Eq. (19) indicates that only target location variable carries the history information inside previous measurements $\mathbf{z}_{1: t-1}$, during the location transition. However, the variables $\mathbf{s}_{t}^{i}$ and $\mathrm{w}_{t}^{i}, \forall i \in \Psi_{t}$, only hold priori information in the state prediction stage.

Hence, the posteriori distribution of the complete state $\boldsymbol{\alpha}_{t}$ is formed as Eq. (20), where the symbol $\propto$ denotes the left is proportional to the right. The posterior distribution $p\left(\boldsymbol{\alpha}_{t} \mid \mathbf{z}_{1: t}\right)$ in Eq. (20) preserves all the necessary information of mobile target tracking. This analysis will assist us in understanding the physical interpretation of the EP analysis of the SLAT scheme in Section V.

\section{B. Calibration Knowledge}

On the other hand, for the location calibration of the $i$ th reference node $\mathbf{s}_{t}^{i}$ (the objective node) of mobile target, we define another $\left(N_{t} D+2 D+N_{t}+1\right)$-dimensional complete variable as $\boldsymbol{\beta}_{t}=\left[\mathbf{s}_{t}^{i} ; \mathbf{x}_{t} ; \mathbf{c}_{t} ; \mathrm{w}_{t}^{i} ; \boldsymbol{\omega}_{t}^{i}\right] .^{6}$ Given the observation $\boldsymbol{y}_{t}$ for the sensor location calibration, the joint likelihood function conditioned on the complete variable $\boldsymbol{\beta}_{t}$ can be written as

$$
\begin{aligned}
p\left(\boldsymbol{y}_{t} \mid \boldsymbol{\beta}_{t}\right)= & \prod_{j \in \Phi_{t}^{i}} \frac{\left|\mathrm{w}_{t}^{i, j}\right|^{\frac{1}{2}}}{\sqrt{2 \pi}} \exp \left(-\frac{1}{2} \mathrm{w}_{t}^{i, j}\left(y_{t}^{i, j}-h\left(\mathbf{s}_{t}^{j}, \mathbf{s}_{t}^{i}\right)\right)^{2}\right) \\
& \cdot \frac{\left|\mathrm{w}_{t}^{i}\right|^{\frac{1}{2}}}{\sqrt{2 \pi}} \exp \left(-\frac{1}{2} \mathrm{w}_{t}^{i}\left(\mathrm{z}_{t}^{i}-h\left(\mathbf{x}_{t}, \mathbf{s}_{t}^{i}\right)\right)^{2}\right),
\end{aligned}
$$

where we assume the measurements $\left\{y_{t}^{i, j} \mid \forall j \in \Phi_{t}^{i}\right\}$ and $\mathrm{z}_{t}^{i}$ are mutually independent. Since all sensor nodes are assumed to be static in the WSN, there is neither transition nor prediction information but the priori information for each node location calibration.

Hence, the posterior distribution of $\boldsymbol{\beta}_{t}$ for sensor location calibration is formed as Eq. (22), where $p\left(\mathbf{x}_{t} \mid \mathbf{z}_{t}\right)$ denotes the marginalized posteriori distribution of the target location $\mathbf{x}_{t}$ that has been tracked, which is

$$
p\left(\mathbf{x}_{t} \mid \mathbf{z}_{t}\right)=\iint p\left(\boldsymbol{\alpha}_{t} \mid \mathbf{z}_{1: t}\right) \mathrm{ds}_{t}^{i} \mathrm{dw}_{t}^{i},
$$

and the posterior density $p\left(\boldsymbol{\alpha}_{t} \mid \mathbf{z}_{1: t}\right)$ is given by Eq. (20).

\section{ERror Propagation Philosophy}

In this section, we will investigate the EP issue of the SLAT problem. This issue comprises of two subproblems, namely, the mobile target tracking EP analysis and the sensor node location calibration EP analysis.

${ }^{6}$ For simplicity, we drop the superscript $i$ in complete state variable $\boldsymbol{\beta}_{t}$ associated with the objective node $\mathbf{s}_{t}^{i}$ whenever no ambiguity arises. 


$$
\begin{aligned}
p\left(\boldsymbol{\alpha}_{t} \mid \mathbf{z}_{1: t}\right) & =\frac{p\left(\mathbf{z}_{t} \mid \boldsymbol{\alpha}_{t}\right) p\left(\boldsymbol{\alpha}_{t} \mid \mathbf{z}_{1: t-1}\right)}{p\left(\mathbf{z}_{t} \mid \mathbf{z}_{1: t-1}\right)} \\
& \propto \mathcal{N}\left(\mathbf{x}_{t} \mid \mathbf{x}_{t-1}^{\sharp}, \Theta_{t}^{p}\right) \prod_{i \in \Psi_{t}} \mathcal{N}\left(\mathrm{z}_{t}^{i} \mid h\left(\mathbf{x}_{t}, \mathbf{s}_{t}^{i}\right), \mathrm{w}_{t}^{i}\right) p\left(\mathbf{s}_{t}^{i} \mid \boldsymbol{\mu}_{t}^{i}, \mathbf{U}_{t}^{i}\right) \mathcal{W}\left(\mathrm{w}_{t}^{i} \mid \Omega, \psi\right)
\end{aligned}
$$

$$
\begin{aligned}
p\left(\boldsymbol{\beta}_{t} \mid \boldsymbol{y}_{t}\right) & =\frac{p\left(\boldsymbol{y}_{t} \mid \boldsymbol{\beta}_{t}\right) p\left(\boldsymbol{\beta}_{t}\right)}{p\left(\boldsymbol{y}_{t}\right)} \\
& \propto \mathcal{N}\left(\mathbf{s}_{t}^{i} \mid \boldsymbol{\mu}_{t}^{i}, \mathbf{U}_{t}^{i}\right) \mathcal{W}\left(\mathrm{w}_{t}^{i} \mid \Omega, \psi\right) \mathcal{W}\left(\mathrm{w}_{t}^{i, j} \mid \Omega, \psi\right) p\left(\mathbf{x}_{t} \mid \mathbf{z}_{t}\right) \cdot \mathcal{N}\left(\mathrm{z}_{t}^{i} \mid h\left(\mathbf{s}_{t}^{i}, \mathbf{x}_{t}\right), \mathrm{w}_{t}^{i}\right) \prod_{j \in \Phi_{t}^{i}} p\left(y_{t}^{i, j} \mid h\left(\mathbf{s}_{t}^{i}, \mathbf{s}_{t}^{j}\right), \mathrm{w}_{t}^{i, j}\right) \mathcal{N}\left(\mathbf{s}_{t}^{j} \mid \boldsymbol{\mu}_{t}^{j}, \mathbf{U}_{t}^{j}\right) .
\end{aligned}
$$

\section{A. Tracking EP Analysis}

1) Bayesian FIM: We focus on the target tracking process at first. Recall the complete state variable $\boldsymbol{\alpha}_{t}$ and the statistical model in Section IV-A. The Bayesian estimation (BE)-based Fisher information matrix (FIM) of $\boldsymbol{\alpha}_{t}$ is given by [26]

$$
\boldsymbol{J}_{\mathrm{BE}}^{\boldsymbol{\alpha}_{t}}=-\mathbb{E}_{\mathbf{z}_{t}, \boldsymbol{\alpha}_{t}}\left\{\nabla_{\boldsymbol{\alpha}_{t}, \boldsymbol{\alpha}_{t}^{\top}} \ln p\left(\boldsymbol{\alpha}_{t} \mid \mathbf{z}_{1: t}\right)\right\}
$$

where $\nabla_{\boldsymbol{\alpha}_{t}, \boldsymbol{\alpha}_{t}^{\top}}(\bullet)$ denotes the second order derivative with respect to $\boldsymbol{\alpha}_{t}$, and $\mathbb{E}_{\mathbf{z}_{t}, \boldsymbol{\alpha}_{t}}\{\bullet\}$ denotes the expectation with respect to the joint distribution $p\left(\mathbf{z}_{t}, \boldsymbol{\alpha}_{t}\right)$.

Let $\mathrm{w}_{t}^{M_{t}}$ denote the measurement precision at the $M_{t}$ th reference node $\mathbf{s}_{t}^{M_{t}}$. We assume each individual state variable $\boldsymbol{\alpha}_{t}^{m} \in\left\{\boldsymbol{\alpha}_{t}\right\}$ is priori independent of each other, where $\boldsymbol{\alpha}_{t}^{m}$ denotes the $m$ th individual state variable in the complete state variable $\boldsymbol{\alpha}_{t}$. Based on statistical model in Section IV, the BE-based FIM in Eq. (24) can be structured as Eq. (25). We can observe that, $\mathcal{I}_{\mathrm{BE}}^{\boldsymbol{\alpha}_{t}^{m}, \boldsymbol{\alpha}_{t}^{n}}=\mathcal{I}_{\mathrm{MLE}}^{\boldsymbol{\alpha}_{t}^{m}, \boldsymbol{\alpha}_{t}^{n}}+\delta_{m, n} \mathcal{I}_{\mathrm{P}}^{\boldsymbol{\alpha}_{t}^{m}}$, $\forall \boldsymbol{\alpha}_{t}^{m}, \boldsymbol{\alpha}_{t}^{n} \in\left\{\boldsymbol{\alpha}_{t}\right\}$, where $\delta_{m, n}=1$ when $m=n$, while $\delta_{m, n}=0$ otherwise. Moreover, the maximum likelihood estimation (MLE)-based FIM $\mathcal{I}_{\text {MLE }}^{\boldsymbol{\alpha}_{t}^{m}, \boldsymbol{\alpha}_{t}^{n}}$ associated with $\boldsymbol{\alpha}_{t}^{m}$ and $\boldsymbol{\alpha}_{t}^{n}\left(\forall \boldsymbol{\alpha}_{t}^{m}, \boldsymbol{\alpha}_{t}^{n} \in\left\{\boldsymbol{\alpha}_{t}\right\}\right)$ and the state prediction-based FIM $\mathcal{I}_{\mathrm{P}}^{\boldsymbol{\alpha}_{t}^{m}}$ associated with $\boldsymbol{\alpha}_{t}^{m}$ are calculated as

$$
\begin{aligned}
\mathcal{I}_{\mathrm{MLE}}^{\boldsymbol{\alpha}_{t}^{m}, \boldsymbol{\alpha}_{t}^{n}} & =-\mathbb{E}_{\mathbf{z}_{t}, \boldsymbol{\alpha}_{t}}\left\{\nabla_{\boldsymbol{\alpha}_{t}^{m}, \boldsymbol{\alpha}_{t}^{n \top}} \ln p\left(\mathbf{z}_{t} \mid \boldsymbol{\alpha}_{t}\right)\right\}, \\
\mathcal{I}_{\mathrm{P}}^{\boldsymbol{\alpha}_{t}^{m}} & =-\mathbb{E}_{\boldsymbol{\alpha}_{t}}\left\{\nabla_{\boldsymbol{\alpha}_{t}^{m}, \boldsymbol{\alpha}_{t}^{m} \top} \ln p\left(\boldsymbol{\alpha}_{t}^{m} \mid \mathbf{z}_{1: t-1}\right)\right\},
\end{aligned}
$$

and $\mathcal{I}_{\mathrm{MLE}}^{\boldsymbol{\alpha}_{t}^{n}, \boldsymbol{\alpha}_{t}^{m}}=\left(\mathcal{I}_{\mathrm{MLE}}^{\boldsymbol{\alpha}_{t}^{m}, \boldsymbol{\alpha}_{t}^{n}}\right)^{\top}$. The involved FIM items can be derived as follows

$$
\begin{aligned}
\mathcal{I}_{\mathrm{MLE}}^{\mathbf{x}_{t}, \mathbf{x}_{t}} & =\sum_{i \in \Psi_{t}} \eta \mathbf{A}_{t}^{i}, \\
\mathcal{I}_{\mathrm{P}}^{\mathbf{x}_{t}} & =\left(\left(\boldsymbol{J}_{\mathrm{BE}}^{\mathbf{x}_{t-1}}\right)^{-1}+\chi^{-1}\right)^{-1}, \\
\mathcal{I}_{\mathrm{MLE}}^{\mathbf{s}_{t}^{i}, \mathbf{x}_{t}} & =-\eta \mathbf{A}_{t}^{i}, \\
\mathcal{I}_{\mathrm{MLE}}^{\mathrm{w}_{t}^{i}, \mathbf{x}_{t}} & =\mathbf{0}_{(1 \times D)}, \\
\mathcal{I}_{\mathrm{MLE}}^{\mathbf{s}_{t}^{i}, \mathbf{s}_{t}^{i}} & =\eta \mathbf{A}_{t}^{i}, \\
\mathcal{I}_{\mathrm{P}}^{\mathbf{s}_{t}^{i}} & =\mathbf{U}_{t}^{i}, \\
\mathcal{I}_{\mathrm{MLE}}^{\mathbf{s}_{t}^{i}, \mathbf{s}_{t}^{j}} & =\mathbf{0}_{(D \times D)}, \text { when } i \neq j .
\end{aligned}
$$

Proof: See APPEndix A, B and C, respectively.

In Eq. (27), $\eta$ denotes the measurement precision factor, while $\mathbf{A}_{t}^{i}$ denotes the geometric-domain resolution factor of measurement system (see Remark 3), which are given by

$$
\begin{aligned}
\eta & =\Omega \psi, \\
\mathbf{A}_{t}^{i} & =\left(\frac{10 \gamma}{\ln 10}\right)^{2} \mathbb{E}_{\mathbf{x}_{t}, \mathbf{s}_{t}^{i}}\left\{\frac{\left(\mathbf{x}_{t}-\mathbf{s}_{t}^{i}\right)\left(\mathbf{x}_{t}-\mathbf{s}_{t}^{i}\right)^{\top}}{\left\|\mathbf{x}_{t}-\mathbf{s}_{t}^{i}\right\|_{2}^{4}}\right\} .
\end{aligned}
$$

Other FIMs associated with measurement precisions, such as $\mathcal{I}_{\mathrm{MLE}}^{\mathrm{w}_{t}^{i}, \mathrm{w}_{t}^{i}}, \mathcal{I}_{\mathrm{MLE}}^{\mathrm{s}_{t}^{i}, \mathrm{w}_{t}^{j}}, \mathcal{I}_{\mathrm{P}}^{\mathrm{w}_{t}^{i}}$ and $\mathcal{I}_{\mathrm{MLE}}^{\mathrm{w}_{t}^{i}, \mathrm{w}_{t}^{j}}$ are not included here, since they will multiply with the all-zero FIM items in Eq. (30).

Furthermore, by letting $\mathcal{I}_{\mathrm{BE}}^{\mathrm{x}_{t}, \mathbf{x}_{t}}=\mathcal{I}_{\mathrm{MLE}}^{\mathrm{x}_{t}, \mathbf{x}_{t}}+\mathcal{I}_{\mathrm{P}}^{\mathrm{x}_{t}}$, the full FIM $J_{\mathrm{BE}}^{\alpha_{t}}$ in Eq. (25) can be partitioned into four sub-matrices

$$
\boldsymbol{J}_{\mathrm{BE}}^{\boldsymbol{\alpha}_{t}}=\left[\begin{array}{cc}
\mathcal{I}_{\mathrm{BE}}^{\mathbf{x}_{t}, \mathbf{x}_{t}} & \left(\mathbf{F}_{\mathrm{BE}}^{\mathbf{x}_{t}}\right)^{\top} \\
\mathbf{F}_{\mathrm{BE}}^{\mathbf{x}_{t}} & \boldsymbol{\mathcal { R }}_{\mathrm{BE}}^{\mathbf{x}_{t}}
\end{array}\right] .
$$

2) Tracking-based EP: The overall Bayesian information of the SLAT problem can be characterized by its BE-based full FIM $\boldsymbol{J}_{\mathrm{BE}}^{\alpha_{t}}$ in Eq. (25), and the equivalent FIM $\boldsymbol{J}_{\mathrm{BE}}^{\mathbf{x}_{t}}$ associated with $\mathbf{x}_{t}$ retains all necessary information for the target tracking, in terms of $\left[\left(\boldsymbol{J}_{\mathrm{BE}}^{\boldsymbol{\alpha}_{t}}\right)^{-1}\right]_{[1: D, 1: D]}=\left(\boldsymbol{J}_{\mathrm{BE}}^{\mathbf{x}_{t}}\right)^{-1}$ [41]. Based on the matrix partition in Eq. (36), the equivalent FIM $\boldsymbol{J}_{\mathrm{BE}}^{\mathrm{x}_{t}}$ can be obtained, using Schur's complement [75], as

$$
\boldsymbol{J}_{\mathrm{BE}}^{\mathbf{x}_{t}}=\mathcal{I}_{\mathrm{BE}}^{\mathbf{x}_{t}, \mathbf{x}_{t}}-\left(\mathbf{F}_{\mathrm{BE}}^{\mathbf{x}_{t}}\right)^{\top}\left(\mathcal{R}_{\mathrm{BE}}^{\mathbf{x}_{t}}\right)^{-1} \mathbf{F}_{\mathrm{BE}}^{\mathbf{x}_{t}}
$$

Combining with Eqs. (27)-(33) and based on the inversive matrix lemma [76], $\boldsymbol{J}_{\mathrm{BE}}^{\mathbf{x}_{t}}$ can be further derived as

$$
\begin{gathered}
\boldsymbol{J}_{\mathrm{BE}}^{\mathbf{x}_{t}}=\mathcal{I}_{\mathrm{P}}^{\mathbf{x}_{t}}+\sum_{i \in \Psi_{t}} \eta \mathbf{A}_{t}^{i}-\underbrace{\sum_{i \in \Psi_{t}} \eta^{2} \mathbf{A}_{t}^{i \top}\left(\eta \mathbf{A}_{t}^{i}+\mathbf{U}_{t}^{i}\right)^{-1} \mathbf{A}_{t}^{i}}_{\begin{array}{c}
\text { The information loss due to } \\
\text { reference node location errors }
\end{array}} \\
=\underbrace{\sum_{i \in \Psi_{t}}\left(\left(\eta \mathbf{A}_{t}^{i}\right)^{-1}+\left(\mathbf{U}_{t}^{i}\right)^{-1}\right)^{-1}}_{\text {Equivalent measurement information } \mathcal{H}_{t}}+\underbrace{\left(\left(\boldsymbol{J}_{\mathrm{BE}}^{\mathbf{x}_{t-1}}\right)^{-1}+\boldsymbol{\chi}^{-1}\right)^{-1}}_{\text {Prediciton information } \mathcal{I}_{\mathrm{P}}^{\mathbf{x}_{t}}} .
\end{gathered}
$$

Proof: See APPEndix D.

In Eq. (39), the error propagation principle associated with mobile target tracking is revealed. In addition, one can see the phenomenon of target tracking error propagation (from $\left(\boldsymbol{J}_{\mathrm{BE}}^{\mathbf{x}_{t}-1}\right)^{-1}$ to $\left.\left(\boldsymbol{J}_{\mathrm{BE}}^{\mathbf{x}_{t}}\right)^{-1}\right)$, based on Eq. (39). The physical interpretation of this tracking-based EP principle will be 


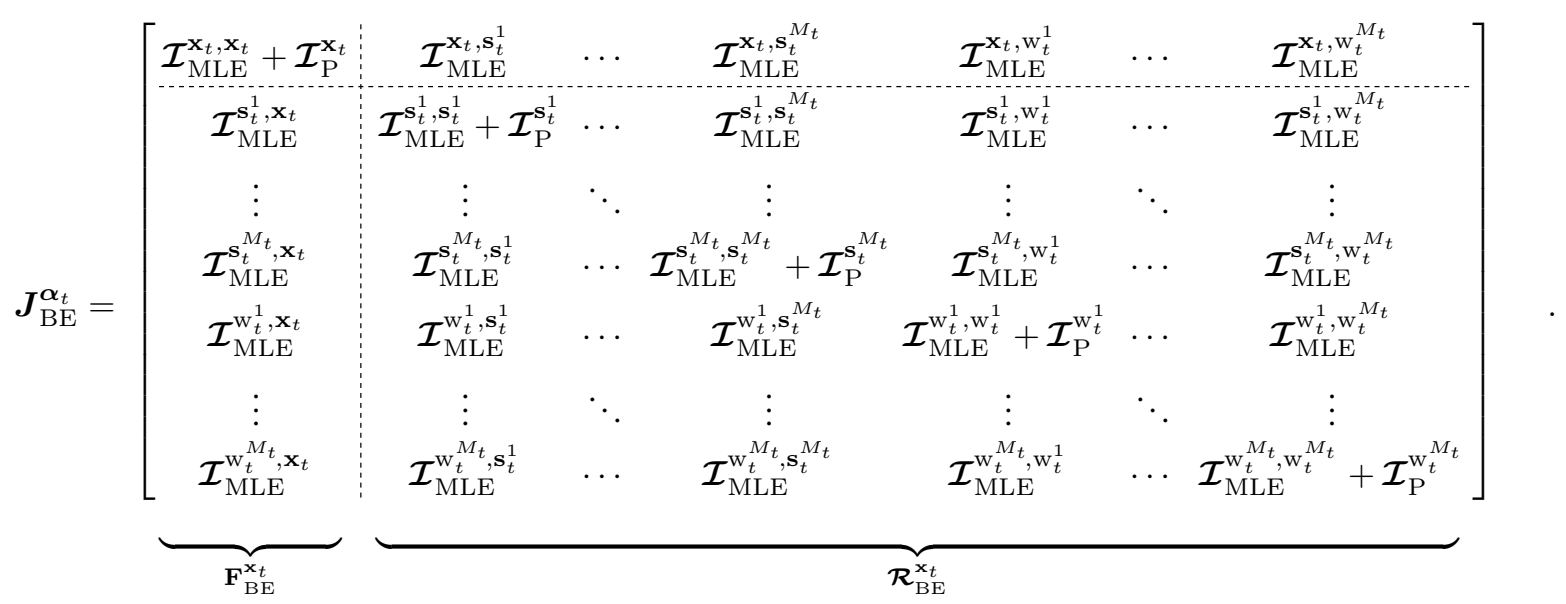

presented in Section VI, and its propagation properties (i.e., the convergence conditions, balance state and robustness) and the asymptotic analysis will be elaborated in Section VII and VIII, respectively.

Remark 1. The location prediction information $\mathcal{I}_{\mathrm{P}}^{\mathbf{x}_{t}}$ and the crude measurement information $\sum_{i \in \Psi_{t}} \eta \mathbf{A}_{t}^{i}$ (that neglects the reference node location error) will contribute to the present tracking accuracy $\boldsymbol{J}_{\mathrm{BE}}^{\mathbf{x}_{t}}$, while the reference node location uncertainties $\left\{\left(\mathbf{U}_{t}^{i}\right)^{-1}: \forall i \in \Psi_{t}\right\}$ will lead to the degradation of $\sum_{i \in \Psi_{t}} \eta^{2} \mathbf{A}_{t}^{i \top}\left(\eta \mathbf{A}_{t}^{i}+\mathbf{U}_{t}^{i}\right)^{-1} \mathbf{A}_{t}^{i}$. As indicated by Eq. (39), the measurement information gained from each sensor node $\mathbf{s}_{t}^{i}$ will be reduced from $\eta \mathbf{A}_{t}^{i}$ to $\left(\left(\eta \mathbf{A}_{t}^{i}\right)^{-1}+\left(\mathbf{U}_{t}^{i}\right)^{-1}\right)^{-1}$, due to the reference sensor node location errors.

Let $\mathcal{H}_{t}^{i}$ denote the equivalent measurement information (inside measurement $\mathrm{z}_{t}^{i}$ ) that the location-inaccurate reference sensor node $\mathbf{s}_{t}^{i}$ actually contributes to the target tracking, which is given by

$$
\mathcal{H}_{t}^{i}=\left(\left(\eta \mathbf{A}_{t}^{i}\right)^{-1}+\left(\mathbf{U}_{t}^{i}\right)^{-1}\right)^{-1}
$$

Then, the overall equivalent measurement information $\mathcal{H}_{t}$ can also be formulated as

$$
\mathcal{H}_{t}=\sum_{i \in \Psi_{t}} \mathcal{H}_{t}^{i}
$$

which indicates the overall localization information gained from the measurements of all location-inaccurate reference sensor nodes $\left\{\mathbf{s}_{t}^{i} \mid \forall i \in \Psi_{t}\right\}$.

Remark 2. As shown in Eq. (38), the mobile tracking information $\boldsymbol{J}_{\mathrm{BE}}^{\mathrm{x}_{t}}$ comes from the prediction information $\mathcal{I}_{\mathrm{P}}^{\mathbf{x}_{t}}$ and the overall equivalent measurement information $\mathcal{H}_{t}$. The equivalent measurement information $\mathcal{H}_{t}^{i}$ benefiting from each reference sensor node $\mathbf{s}_{t}^{i}$ is determined by the crude measurement information $\eta \mathbf{A}_{t}^{i}$ and the reference node location's prior precision $\mathbf{U}_{t}^{i}$. Besides, the prediction information is formed by the latest posterior precision $\boldsymbol{J}_{\mathrm{BE}}^{\mathbf{x}_{t-1}}$ and transition precision $\chi$.

Remark 3. It is interesting to note that both the measurement resolution factor $\mathbf{A}_{t}^{i}$ and the measurement precision factor $\eta$ contribute to the coarse measurement information $\eta \mathbf{A}_{t}^{i}$ [10], [77]. The measurement resolution factor $\mathbf{A}_{t}^{i}$ indicates the discrimination capability associated with the measurement system that the tracking system precisely recognizes the location difference, given a certain measurement variation. While, the inverse of measurement precision factor $\eta$ implies the extent that the measurement noises blur the location estimation. An illustration of the resolution factor and the precision factor of the measurement system is given in Fig. 2.

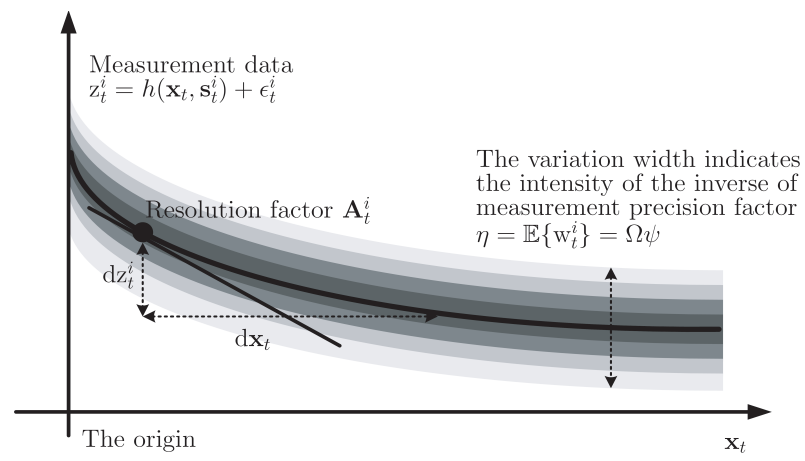

Fig. 2. The resolution and precision factors of the measurement system.

Remark 4. From Eq. (39) we know that, the mobile tracking accuracy $\boldsymbol{J}_{\mathrm{BE}}^{\mathbf{x}_{t}}$ is completely determined by the equivalent measurement information $\left\{\mathcal{H}_{t}^{i} \mid \forall i \in \Psi_{t}\right\}$ and prediction information $\mathcal{I}_{\mathrm{P}}^{\mathbf{x}_{t}}$, i.e., $\boldsymbol{J}_{\mathrm{BE}}^{\mathbf{x}_{t}}=\sum_{i \in \Psi_{t}} \mathcal{H}_{t}^{i}+\mathcal{I}_{\mathrm{P}}^{\mathbf{x}_{t}}$. In addition, the prediction information $\mathcal{I}_{\mathrm{P}}^{\mathrm{x}_{t}}$ is entirely dominated by the mobile target state, while the equivalent measurement information $\mathcal{H}_{t}^{i}$ is dependent on the measurement choice, measurement state (measurement precision $\eta$ ) and the reference node state $\left(\boldsymbol{\mu}_{t}^{i}\right.$ and $\mathbf{U}_{t}^{i}$ ). In order to improve the tracking performance, efficient sensor network deployment [48], [49], [53], tracking algorithm design and promising measurement choice can be conducted based on Eq. (39). In an energy-constrained wireless sensor network [18], [19], based on the relationship between $\mathcal{H}_{t}^{i}$ and the state of $\mathbf{s}_{t}^{i}$, either a reasonable reference sensor node selection strategy [21], [51], [52], [53] or power allocation scheme [47] is needed to strike the balance between energy consumption and computational overhead.

Based on the equivalent FIM expressed in Eq. (39), the 
CRLB associated with target tracking is given by

$$
\mathcal{B}_{\mathrm{BE}}^{\mathbf{x}_{t}}=\left(\boldsymbol{J}_{\mathrm{BE}}^{\mathbf{x}_{t}}\right)^{-1}=\left(\sum_{i \in \Psi_{t}} \mathcal{H}_{t}^{i}+\mathcal{I}_{\mathrm{P}}^{\mathbf{x}_{t}}\right)^{-1}
$$

where $\mathcal{H}_{t}^{i}$ is defined in Eq. (40). This implies that the tracking error $\left\|\mathbf{x}_{t}-\widehat{\mathbf{x}}_{t}\right\|_{2}$ is bounded by $\left\|\mathrm{x}_{t}-\widehat{\mathbf{x}}_{t}\right\|_{2} \geq\left(\operatorname{tr}\left(\mathcal{B}_{\mathrm{BE}}^{\mathbf{x}_{t}}\right)\right)^{\frac{1}{2}}$, where $\widehat{\mathbf{x}}_{t}$ denotes the target location estimation, and $\operatorname{tr}(\bullet)$ denotes the matrix trace.

\section{B. Calibration EP Analysis}

1) Bayesian FIM: Now we focus on the location calibration of the reference node $\mathbf{s}_{t}^{i}$ (the objective node).

According to the calibration process introduced in Section III, other $N_{t}$ nodes nearby and the target together form a new reference cluster to calibrate the objective node $\mathbf{s}_{t}^{i}$. Recalling the associated complete variable $\boldsymbol{\beta}_{t}$ and the statistical model in Section IV-B, the full FIM $\boldsymbol{J}_{\mathrm{BE}}^{\boldsymbol{\beta}_{t}}$ for the objective node location calibration can be derived as ${ }^{7}$

$$
\begin{aligned}
\boldsymbol{J}_{\mathrm{BE}}^{\boldsymbol{\beta}_{t}} & =-\mathbb{E}_{\boldsymbol{y}_{t}, \boldsymbol{\beta}_{t}}\left\{\nabla_{\boldsymbol{\beta}_{t}, \boldsymbol{\beta}_{t}^{\top}} \ln p\left(\boldsymbol{\beta}_{t} \mid \boldsymbol{y}_{1: t}\right)\right\} \\
& =\left[\begin{array}{cc}
\mathcal{I}_{\mathrm{BE}}^{\mathbf{S}_{t}^{i}, \mathbf{s}_{t}^{i}} & \left(\mathbf{F}_{\mathrm{BE}}^{\mathbf{s}_{t}^{i}}\right)^{\top} \\
\mathbf{F}_{\mathrm{BE}}^{\mathbf{s}_{t}^{i}} & \boldsymbol{\mathcal { R }}_{\mathrm{BE}}^{\mathbf{s}_{t}^{i}}
\end{array}\right],
\end{aligned}
$$

where the submatrices $\mathbf{F}_{\mathrm{BE}}^{\mathbf{s}_{t}^{i}}$ and $\mathcal{R}_{\mathrm{BE}}^{\mathbf{s}_{t}^{i}}$ are

$$
\begin{aligned}
\mathcal{I}_{\mathrm{BE}}^{\mathbf{s}_{t}^{i}, \mathbf{s}_{t}^{i}} & =\mathcal{I}_{\mathrm{MLE}}^{\mathbf{s}_{t}^{i}, \mathbf{s}_{t}^{i}}+\mathcal{I}_{\mathrm{P}}^{\mathbf{s}_{t}^{i}, \mathbf{s}_{t}^{i}}, \\
\mathbf{F}_{\mathrm{BE}}^{\mathbf{s}_{t}^{i}} & =\operatorname{vec}\left[\mathcal{I}_{\mathrm{MLE}}^{\boldsymbol{\beta}_{t}^{n}, \mathbf{s}_{t}^{i}}\right]_{\forall n: \boldsymbol{\beta}_{t}^{n} \neq \mathbf{s}_{t}^{i},} \\
\mathcal{R}_{\mathrm{BE}}^{\mathbf{s}_{t}^{i}} & =\operatorname{mat}\left[\mathcal{I}_{\mathrm{BE}}^{\boldsymbol{\beta}_{t}^{m}, \boldsymbol{\beta}_{t}^{n}}\right]_{\forall m, n: \boldsymbol{\beta}_{t}^{m}, \boldsymbol{\beta}_{t}^{n} \neq \mathbf{s}_{t}^{i}} \\
& =\operatorname{mat}\left[\mathcal{I}_{\mathrm{MLE}}^{\boldsymbol{\beta}_{t}^{m}, \boldsymbol{\beta}_{t}^{n}}+\delta_{m, n} \mathcal{I}_{\mathrm{P}}^{\boldsymbol{\beta}_{t}^{m}}\right]_{\forall m, n: \boldsymbol{\beta}_{t}^{m}, \boldsymbol{\beta}_{t}^{n} \neq \mathbf{s}_{t}^{i}} .
\end{aligned}
$$

Moreover, $\forall \boldsymbol{\beta}_{t}^{m}, \boldsymbol{\beta}_{t}^{n} \in\left\{\boldsymbol{\beta}_{t}\right\}$, the MLE-based FIM $\mathcal{I}_{\text {MLE }}^{\boldsymbol{\beta}_{t}^{m}, \boldsymbol{\beta}_{t}^{n}}$ and the priori FIM $\mathcal{I}_{\mathrm{P}}^{\boldsymbol{\beta}_{t}^{m}}$ can be calculated as

$$
\begin{aligned}
\mathcal{I}_{\mathrm{MLE}}^{\boldsymbol{\beta}_{t}^{m}, \boldsymbol{\beta}_{t}^{n}} & =-\mathbb{E}_{\boldsymbol{y}_{t}, \boldsymbol{\beta}_{t}}\left\{\nabla_{\boldsymbol{\beta}_{t}^{m}, \boldsymbol{\beta}_{t}^{n \top}} \ln p\left(\boldsymbol{y}_{t} \mid \boldsymbol{\beta}_{t}\right)\right\}, \\
\mathcal{I}_{\mathrm{P}}^{\boldsymbol{\beta}_{t}^{m}} & =-\mathbb{E}_{\boldsymbol{\beta}_{t}}\left\{\nabla_{\boldsymbol{\beta}_{t}^{m}, \boldsymbol{\beta}_{t}^{m} \top} \ln p\left(\boldsymbol{\beta}_{t}^{m}\right)\right\} .
\end{aligned}
$$

Here, vec $\left[\bullet_{n}\right]_{\forall n: \boldsymbol{\beta}_{t}^{n} \neq \mathbf{s}_{t}^{i}}$ stacks all elements $\bullet_{n}$ in columns, $\forall n$

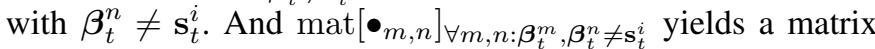
whose $(m, n)$ th component is $\bullet_{m, n}, \forall m, n$ where $\boldsymbol{\beta}_{t}^{m}, \boldsymbol{\beta}_{t}^{n} \neq$ $\mathbf{s}_{t}^{i}$. In the same way, $\forall \boldsymbol{\beta}_{t}^{m}, \boldsymbol{\beta}_{t}^{n} \in\left\{\boldsymbol{\beta}_{t}\right\}$, all the involved FIM items of $\mathcal{I}_{\mathrm{MLE}}^{\boldsymbol{\beta}_{t}^{m}, \boldsymbol{\beta}_{t}^{n}}$ and $\mathcal{I}_{\mathrm{P}}^{\boldsymbol{\beta}_{t}^{m}}$ are given by

$$
\begin{aligned}
\mathcal{I}_{\mathrm{MLE}}^{\mathbf{s}_{t}^{i}, \mathbf{s}_{t}^{i}} & =\sum_{j \in \Phi_{t}^{i}} \eta \mathbf{A}_{t}^{i, j}+\eta \mathbf{A}_{t}^{i}, \\
\mathcal{I}_{\mathrm{P}}^{\mathbf{s}_{t}^{i}} & =\mathbf{U}_{t}^{i}, \\
\mathcal{I}_{\mathrm{MLE}}^{\mathbf{x}_{t}, \mathbf{s}_{t}^{i}} & =-\eta \mathbf{A}_{t}^{i}, \\
\mathcal{I}_{\mathrm{MLE}}^{\mathbf{s}_{t}^{j}, \mathbf{s}_{t}^{i}} & =-\eta \mathbf{A}_{t}^{i, j}, \forall j \in \Phi_{t}^{i}, \\
\mathcal{I}_{\mathrm{MLE}}^{\mathrm{w}_{t}^{i, j}, \mathbf{s}_{t}^{i}} & =\mathcal{I}_{\mathrm{MLE}}^{\mathrm{w}_{t}^{i}, \mathbf{s}_{t}^{i}}=\mathbf{0}_{(1 \times D)}, \forall j \in \Phi_{t}^{i}, \\
\mathcal{I}_{\mathrm{MLE}}^{\mathbf{x}_{t}, \mathbf{x}_{t}} & =\eta \mathbf{A}_{t}^{i}, \\
\mathcal{I}_{\mathrm{P}}^{\mathbf{x}_{t}} & =\boldsymbol{J}_{\mathrm{BE}}^{\mathbf{x}_{t}},
\end{aligned}
$$

${ }^{7}$ Please refer to the structure of $\boldsymbol{\mathcal { R }}_{\mathrm{BE}}^{\mathbf{x}_{t}}$ in Eqs. (25) and (36).

$$
\begin{aligned}
\mathcal{I}_{\mathrm{MLE}}^{\mathbf{s}_{t}^{j}, \mathbf{x}_{t}} & =\mathbf{0}, \forall j \in \Phi_{t}^{i}, \\
\mathcal{I}_{\mathrm{MLE}}^{\mathbf{s}_{t}^{j}, \mathbf{s}_{t}^{j}} & =\eta \mathbf{A}_{t}^{i, j}, \forall j \in \Phi_{t}^{i}, \\
\mathcal{I}_{\mathrm{P}}^{\mathbf{s}_{t}^{j}} & =\mathbf{U}_{t}^{j}, \forall j \in \Phi_{t}^{i}, \\
\mathcal{I}_{\mathrm{MLE}}^{\mathbf{s}_{t}^{k}, \mathbf{s}_{t}^{j}} & =\mathbf{0}, \forall j, k \in \Phi_{t}^{i} \text { and } j \neq k, \\
\mathcal{I}_{\mathrm{MLE}}^{\mathrm{w}_{t}^{i}, \mathbf{x}_{t}} & =\mathcal{I}_{\mathrm{MLE}}^{\mathrm{w}_{t}^{i, j}, \mathbf{x}_{t}}=\boldsymbol{I}_{\mathrm{MLE}}^{\mathrm{w}_{t}^{i}, \mathbf{s}_{t}^{j}} \\
& =\mathcal{I}_{\mathrm{MLE}}^{\mathrm{w}_{t}^{i, k}, \mathbf{s}_{t}^{j}}=\mathbf{0}_{(1 \times D)}, \forall j, k \in \Phi_{t}^{i},
\end{aligned}
$$

where the geometric-domain resolution factor $\mathbf{A}_{t}^{i, j}$ of measurement associated with sensor nodes $\mathbf{s}_{t}^{i}$ and $\mathbf{s}_{t}^{j}$ is given by

$$
\mathbf{A}_{t}^{i, j}=\left(\frac{10 \gamma}{\ln 10}\right)^{2} \mathbb{E}_{\mathbf{s}_{t}^{i}, \mathbf{s}_{t}^{j}}\left\{\frac{\left(\mathbf{s}_{t}^{j}-\mathbf{s}_{t}^{i}\right)\left(\mathbf{s}_{t}^{j}-\mathbf{s}_{t}^{i}\right)^{\top}}{\left\|\mathbf{s}_{t}^{j}-\mathbf{s}_{t}^{i}\right\|_{2}^{4}}\right\} .
$$

Proof: The derivation of Eqs. (49)-(61) is similar to those of Eqs. (27)-(35) in Appendix A, B and C.

The measurement resolution factor $\mathbf{A}_{t}^{i, j}$ associated with sensor node location calibration indicates the discrimination ability of a localization scheme, which has a similar physical connotation with $\mathbf{A}_{t}^{i}$, as revealed in Remark $\mathbf{3}$.

Other FIMs associated with measurement precisions, such as $\mathcal{I}_{\mathrm{MLE}}^{\mathrm{w}_{t}^{i}, \mathrm{w}_{t}^{i}}, \mathcal{I}_{\mathrm{MLE}}^{\mathrm{w}_{t}^{i, j}, \mathrm{w}_{t}^{i, j}}, \mathcal{I}_{\mathrm{P}}^{\mathrm{w}_{t}^{i, j}}, \mathcal{I}_{\mathrm{P}}^{\mathrm{w}_{t}^{i}}$ and $\mathcal{I}_{\mathrm{MLE}}^{\mathrm{w}_{t}^{i}, \mathrm{w}_{t}^{i, j}}, \mathcal{I}_{\mathrm{MLE}}^{\mathrm{w}_{t}^{i, k}, \mathrm{w}_{t}^{i, j}}$, $\forall j, k \in \Phi_{t}^{i}$, are not included here, since they will multiply with the all-zero FIM items in Eq. (60).

2) Calibration-based EP: Given FIMs in Eqs. (49)-(60), and by using Schur's complement [75] and the inversive matrix lemma [76], the equivalent BE-based FIM $\boldsymbol{J}_{\mathrm{BE}}^{\mathbf{s}_{t}^{i}}$ associated with calibration can be derived as in Eq. (63).

Proof: The derivation of Eq. (63) is similar to that of Eq. (39) in APPENDIX D.

The error propagation principle associated with the sensor node location calibration is revealed in Eq. (63). Correspondingly, one can observe the error propagation behavior, from $\left(\mathbf{U}_{t}^{i}\right)^{-1}$ to $\left(\boldsymbol{J}_{\mathrm{BE}}^{\mathbf{s}_{t}^{i}}\right)^{-1}$, based on Eq. (63). The physical interpretation of the calibration EP principle, the EP properties and its asymptotic analysis will be elaborated in Section VI, VII and VIII, respectively.

Remark 5. It can be observed from Eq. (63) that, the current calibration accuracy $\boldsymbol{J}_{\mathrm{BE}}^{\mathbf{s}_{t}^{i}}$ is completely determined by (1) the equivalent measurement information $\mathcal{H}_{t}^{\mathbf{s}}=\sum_{j \in \Phi_{t}^{i}}\left(\left(\eta \mathbf{A}_{t}^{i, j}\right)^{-1}+\right.$ $\left.\left(\mathbf{U}_{t}^{j}\right)^{-1}\right)^{-1}$ from its $N_{t}$ nearby sensor nodes, (11) the equivalent measurement information $\mathcal{H}_{t}^{\mathbf{x}}=\left(\left(\eta \mathbf{A}_{t}^{i}\right)^{-1}+\left(\mathcal{I}_{\mathrm{BE}}^{\mathbf{x}_{t}}\right)^{-1}\right)^{-1}$ from the localized target and (111) its own priori state $\mathbf{U}_{t}^{i}$. In addition, the information loss due to the limited sensor node location precision is $\eta^{2}\left(\mathbf{A}_{t}^{i, j}\right)^{\top}\left(\eta \mathbf{A}_{t}^{i, j}+\mathbf{U}_{t}^{j}\right)^{-1} \mathbf{A}_{t}^{i, j}$, while the information loss from the limited mobile tracking accuracy is $\eta^{2} \mathbf{A}_{t}^{i \top}\left(\eta \mathbf{A}_{t}^{i}+\boldsymbol{J}_{\mathrm{BE}}^{\mathbf{x}_{t}}\right)^{-1} \mathbf{A}_{t}^{i}$. Also due to the errors in sensor node locations and mobile tracking, the measurement information is reduced from the coarse states $\sum_{j \in \Phi_{t}^{i}} \eta \mathbf{A}_{t}^{i, j}$ and $\eta \mathbf{A}_{t}^{i}$ to the equivalent states $\mathcal{H}_{t}^{\mathrm{s}}$ and $\mathcal{H}_{t}^{\mathrm{x}}$, respectively.

The CRLB associated with the sensor node location calibration can thus be derived as

$$
\mathcal{B}_{\mathrm{BE}}^{\mathbf{s}_{t}^{i}}=\left(\boldsymbol{J}_{\mathrm{BE}}^{\mathbf{s}_{t}^{i}}\right)^{-1} .
$$




$$
\begin{aligned}
& \boldsymbol{J}_{\mathrm{BE}}^{\mathbf{s}_{t}^{i}}=\mathcal{I}_{\mathrm{MLE}}^{\mathbf{s}_{t}^{i}, \mathbf{s}_{t}^{i}}+\mathcal{I}_{\mathrm{P}}^{\mathbf{s}_{t}^{i}}-\left(\mathbf{F}_{\mathrm{BE}}^{\mathbf{s}_{t}^{i}}\right)^{\top}\left(\boldsymbol{\mathcal { R }}_{\mathrm{BE}}^{\mathbf{s}_{t}^{i}}\right)^{-1} \mathbf{F}_{\mathrm{BE}}^{\mathbf{s}_{t}^{i}} \\
& =\sum_{j \in \Phi_{t}^{i}} \eta \mathbf{A}_{t}^{i, j}-\sum_{j \in \Phi_{t}^{i}} \underbrace{\eta^{2}\left(\mathbf{A}_{t}^{i, j}\right)^{\top}\left(\eta \mathbf{A}_{t}^{i, j}+\mathbf{U}_{t}^{j}\right)^{-1} \mathbf{A}_{t}^{i, j}}_{\begin{array}{c}
\text { Information loss due to } \\
\text { reference node location error }
\end{array}}+\eta \mathbf{A}_{t}^{i}-\underbrace{\eta^{2} \mathbf{A}_{t}^{i \top}\left(\eta \mathbf{A}_{t}^{i}+\boldsymbol{J}_{\mathrm{BE}}^{\mathbf{x} t}\right)^{-1} \mathbf{A}_{t}^{i}}_{\begin{array}{c}
\text { Information loss due to } \\
\text { mobile target tracking error }
\end{array}}+\mathbf{U}_{t}^{i}, \\
& =\underbrace{\sum_{j \in \Phi_{t}^{i}}\left(\left(\eta \mathbf{A}_{t}^{i, j}\right)^{-1}+\left(\mathbf{U}_{t}^{j}\right)^{-1}\right)^{-1}}_{\mathcal{H}^{\mathbf{s}}}+\underbrace{\left(\left(\eta \mathbf{A}_{t}^{i}\right)^{-1}+\left(\boldsymbol{J}_{\mathrm{BE}}^{\mathbf{x}_{t}}\right)^{-1}\right)^{-1}}_{\text {Equivalent measurement information }}+\mathbf{U}_{t}^{i} . \\
& \mathcal{H}_{t}^{\mathbf{s}} \\
& \text { Equivalent measurement information } \\
& \text { from sensor nodes }
\end{aligned}
$$

This means that, the calibration error $\left\|\mathbf{s}_{t}^{i}-\widehat{\mathbf{s}}_{t}^{i}\right\|_{2}$ can be lower bounded by $\left\|\widehat{\mathbf{s}}_{t}^{i}-\mathbf{s}_{t}^{i}\right\|_{2} \geq\left(\operatorname{tr}\left(\mathcal{B}_{\mathrm{BE}}^{\mathbf{s}_{t}^{i}}\right)\right)^{\frac{1}{2}}$, where $\widehat{\mathbf{s}}_{t}^{i}$ denotes the location estimation of node $\mathbf{s}_{t}^{i}$.

\section{Physical Interpretation OF The EP Philosophy}

\section{A. Interpretation of $E P$}

We use the concept of the fundamental electronics in circuit theory to present an intuitive physical interpretation of both target tracking EP and node location calibration EP principles.

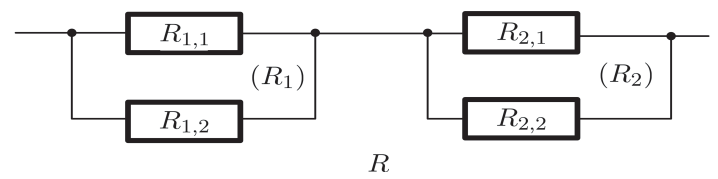

Fig. 3. Illustration of the Ohm's Law.

Let us consider an electronic circuit with resistances connected in parallel and in series, as shown in Fig. 3. Based on the Ohm's Law, the equivalent resistance of this circuit is given by

$$
\begin{aligned}
R & =\underbrace{\left(R_{1,1}^{-1}+R_{1,2}^{-1}\right)^{-1}}_{R_{1}}+\underbrace{\left(R_{2,1}^{-1}+R_{2,2}^{-1}\right)^{-1}}_{R_{2}} \\
& =R_{1}+R_{2} .
\end{aligned}
$$

By comparing the tracking EP in Eq. (39) and the calibration EP in Eq. (63) with Eq. (65), it is evident that both EP characteristics comply with the Ohm's Law.

Remark 6. The previous tracking precision $\boldsymbol{J}_{\mathrm{BE}}^{\mathbf{x}_{t-1}}$ and the target transition precision $\chi$ can be equivalently deemed as resistances connected in parallel, forming the current prediction information $\boldsymbol{J}_{\mathrm{P}}^{\mathbf{x}_{t}}$; The coarse measurement information $\eta \mathbf{A}_{t}^{i}$ and the location precision $\mathbf{U}_{t}^{i}$ of reference node $\mathbf{s}_{t}^{i}$ can also be deemed as the resistances connected in parallel, forming the equivalent measurement information $\mathcal{H}_{t}^{i}$. These equivalent measurement information $\left\{\mathcal{H}_{t}^{i}: \forall i \in \Psi_{t}\right\}$ from all current reference nodes and the current prediction information $\boldsymbol{J}_{\mathrm{P}}^{\mathbf{x}_{t}}$ propagate like the resistances connected in series (resistance summation), forming the overall tracking accuracy $\boldsymbol{J}_{\mathrm{BE}}^{\mathbf{x}_{t}}$ at current time instant.

In fact, according to the Bayesian statistics of SLAT, the measurement information and the prediction information are two independent information sources, both contributing to the mobile target tracking performance. However, due to the uncertainties of reference node locations, the equivalent measurement information $\mathcal{H}_{t}^{i}$ is reduced from $\eta \mathbf{A}_{t}^{i}$ to $\left(\left(\eta \mathbf{A}_{t}^{i}\right)^{-1}+\left(\mathbf{U}_{t}^{i}\right)^{-1}\right)^{-1}$, which resembles a parallel circuit with two resistances of $R_{1,1}=\eta \mathbf{A}_{t}^{i}$ and $R_{1,2}=\mathbf{U}_{t}^{i}$, as illustrated in Fig. 4. Similarly, due to the randomness of the target transition, the latest target tracking information $\boldsymbol{J}_{\mathrm{BE}}^{\mathbf{x}_{t-1}}$ is reduced by $\chi$, forming the prediction information $\mathcal{I}_{\mathrm{P}}^{\mathbf{x}_{t}}$. This also resembles a parallel circuit with two resistances of $R_{2,1}=\boldsymbol{J}_{\mathrm{BE}}^{\mathbf{x}_{t-1}}$ and $R_{2,2}=\boldsymbol{\chi}$, as illustrated in Fig. 4.

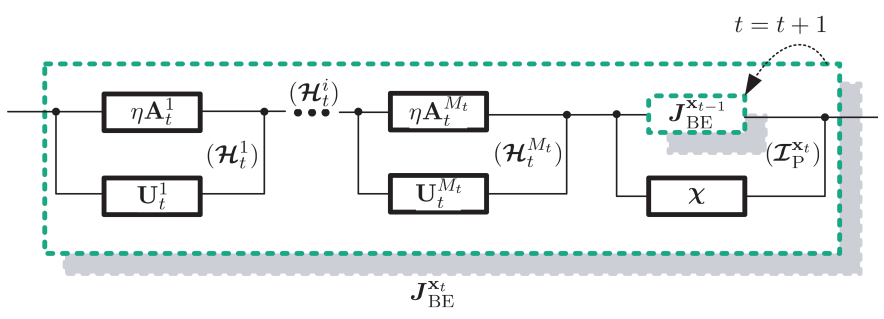

Fig. 4. Illustration of the mobile target tracking EP principle.

It should be noted that, when there is a different priori model assumed for the measurement precision $\mathrm{w}_{t}^{i}$ (instead of the Wishart model in Eqs. (9) and (10)), one can derive the same form of tracking EP principle and calibration EP principle as Eqs. (39) and (63), respectively. The only difference lies in the expression of measurement precision factor $\eta$ (see Eq. (34)).

The above physical interpretation not only presents an intuitive way to capture all factors which dominate the EP philosophy of the SLAT problem, but also provides important insights into the SLAT performance limits.

One may also see that, the tracking information $\boldsymbol{J}_{\mathrm{BE}}^{\mathbf{x}_{t}}$ will finally converge to a balance state when the localization information reduction due to the limited target transition precision $\chi$ equals to the overall equivalent measurement precision $\sum_{i \in \Psi_{t}} \mathcal{H}_{t}^{i}$. In fact, the balance state of $\boldsymbol{J}_{\mathrm{BE}}^{\mathbf{x}_{t}}$ changes over time due to the alternation of reference node members of mobile target, as will be elaborated in Section VII, where its convergence properties will also be discussed.

\section{B. Generalized EP in Filtering Problem}

Here, we extend the above EP principles and their physical interpretation to the general filtering problem.

1) Linear and Gaussian Filtering: Recall a classical linear and Gaussian filtering system, where the observation function 
and the state transition function are, respectively, given by

$$
\begin{aligned}
& \mathbf{z}_{t}=\mathcal{Q}_{t} \mathbf{x}_{t}+\boldsymbol{\epsilon}_{t}, \\
& \mathbf{x}_{t}=\mathcal{P}_{t} \mathbf{x}_{t-1}+\boldsymbol{\varsigma}_{t},
\end{aligned}
$$

where $\mathbf{z}_{t}$ stands for the corresponding $M$-dimensional measurement vector, $\mathcal{Q}_{t}$ is the measurement coefficient matrix, and $\mathcal{P}_{t}$ is the transition coefficient matrix. In addition, $\boldsymbol{\epsilon}_{t}$ and $\boldsymbol{s}_{t}$ are the measurement noise and the transition noise, respectively, which are generally assumed to be Gaussian, i.e. $\boldsymbol{\epsilon}_{t} \sim \mathcal{N}\left(\boldsymbol{\epsilon}_{t} \mid \mathbf{0}, \mathbf{W}\right)$ and $\boldsymbol{\varsigma}_{t} \sim \mathcal{N}\left(\boldsymbol{\varsigma}_{t} \mid \mathbf{0}, \boldsymbol{\chi}\right)$, where $\mathbf{W}$ and $\chi$ are the associated precision matrices. The final filtering error can be formulated as [78]

$$
\begin{aligned}
\mathcal{B}_{\mathrm{BE}}^{\mathbf{x}_{t}} & =\left(\mathcal{I}_{\mathrm{P}}^{\mathbf{x}_{t}}\right)^{-1}-\boldsymbol{H}_{t} \mathcal{Q}_{t}\left(\mathcal{I}_{\mathrm{P}}^{\mathbf{x}_{t}}\right)^{-1}, \\
\boldsymbol{H}_{t} & =\left(\mathcal{I}_{\mathrm{P}}^{\mathbf{x}_{t}}\right)^{-1} \mathcal{Q}_{t}^{\top} \boldsymbol{G}_{t}^{-1}, \\
\boldsymbol{G}_{t} & =\mathcal{Q}_{t}\left(\mathcal{I}_{\mathrm{P}}^{\mathbf{x}_{t}}\right)^{-1} \mathcal{Q}_{t}^{\top}+\mathbf{W}^{-1}, \\
\left(\mathcal{I}_{\mathrm{P}}^{\mathbf{x}_{t}}\right)^{-1} & =\mathcal{P}_{t} \mathcal{B}_{\mathrm{BE}}^{\mathbf{x}_{t-1}} \mathcal{P}_{t}^{\top}+\chi^{-1} .
\end{aligned}
$$

By using the inversive matrix lemma, the filtering accuracy can be further derived in a more concise form as

$$
\begin{aligned}
\boldsymbol{J}_{\mathrm{BE}}^{\mathbf{x}_{t}} & =\mathcal{Q}_{t}^{\top} \mathbf{W} \mathcal{Q}_{t}+\mathcal{I}_{\mathrm{P}}^{\mathbf{x}_{t}} \\
& =\mathcal{Q}_{t}^{\top} \mathbf{W} \mathcal{Q}_{t}+\left(\mathcal{P}_{t}\left(\boldsymbol{J}_{\mathrm{BE}}^{\mathbf{x}_{t-1}}\right)^{-1} \mathcal{P}_{t}^{\top}+\chi^{-1}\right)^{-1},
\end{aligned}
$$

where $\mathcal{Q}_{t}^{\top} \mathbf{W} \mathcal{Q}_{t}=\mathcal{H}_{t}$ denotes the equivalent measurement information accounting for the linear measurement matrix $\mathcal{Q}_{t}$.

Proof: Refer to APPENDIX E.

Remark 7. The EP principle of linear \& Gaussian filtering in Eq. (72) complies with the aforementioned mobile trackingassociated EP philosophy, as shown in Fig. 5. Since there is no reference uncertainty $\left(\mathbf{U}_{t}^{i}\right)^{-1}$ assumed in this filtering problem, the EP of linear \& Gaussian filtering system is a special case of the mobile tracking-associated EP principles (see Eqs. (39) or (91)). In addition, due to the existence of linear coefficients, the measurement information intensity is expanded or constricted quadratically.

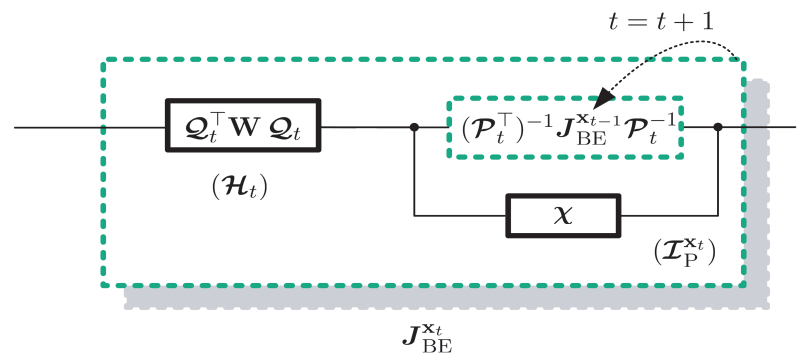

Fig. 5. Illustration of the EP principle of linear and Gaussian filtering, where there is no reference variable uncertainties assumed.

2) Nonlinear and Non-Gaussian Filtering: Now we consider the nonlinear and non-Gaussian filtering as

$$
\begin{aligned}
& \mathbf{z}_{t}=\boldsymbol{h}\left(\mathbf{x}_{t}\right)+\boldsymbol{\epsilon}_{t}, \\
& \mathbf{x}_{t}=\boldsymbol{\ell}\left(\mathbf{x}_{t-1}\right)+\boldsymbol{\varsigma}_{t},
\end{aligned}
$$

where the measurement function $\boldsymbol{h}\left(\mathbf{x}_{t}\right)$ and the transition function $\ell\left(\mathbf{x}_{t-1}\right)$ are both nonlinear. In addition, the measurement noise $\epsilon_{t}$ and the state transition noise $\varsigma_{t}$ are not assumed to have a Gaussian priori distribution.
For the non-Gaussian measurement noise vector $\epsilon_{t}$, the Laplace approximation method can be applied [71], [79] to approximate it as a Gaussian random vector, i.e.,

$$
p\left(\boldsymbol{\epsilon}_{t}\right) \approx \mathcal{N}\left(\boldsymbol{\epsilon}_{t} \mid \mathbf{0}, \mathbf{W}\right),
$$

where $\mathbf{W}$ denotes the approximate precision matrix, i.e.,

$$
\mathbf{W}=\left.\nabla_{\boldsymbol{\epsilon}_{t}, \boldsymbol{\epsilon}_{t}^{\top}} \ln p\left(\boldsymbol{\epsilon}_{t}\right)\right|_{\boldsymbol{\epsilon}_{t}=\mathbf{0}} .
$$

Hence, the measurement information $\mathcal{I}_{\mathrm{MLE}}^{\mathrm{x}_{t}, \mathrm{x}_{t}}$ is given by

$$
\begin{aligned}
\mathcal{I}_{\mathrm{MLE}}^{\mathbf{x}_{t}, \mathbf{x}_{t}} & =-\mathbb{E}_{\mathbf{z}_{t}, \mathbf{x}_{t}}\left\{\nabla_{\mathbf{x}_{t}, \mathbf{x}_{t}^{\top}} \ln \mathcal{N}\left(\mathbf{z}_{t} \mid \boldsymbol{h}\left(\mathbf{x}_{t}\right), \mathbf{W}\right)\right\} \\
& =\left\langle\mathcal{Q}_{t}^{\top} \mathbf{W} \mathcal{Q}_{t}\right\rangle_{\mathbf{x}_{t}},
\end{aligned}
$$

where $\left\langle\boldsymbol{y}\left(\mathbf{x}_{t}\right)\right\rangle_{\mathbf{x}_{t}}=\mathbb{E}_{\mathbf{x}_{t}}\left\{\boldsymbol{y}\left(\mathbf{x}_{t}\right)\right\}=\int \boldsymbol{y}\left(\mathbf{x}_{t}\right) p\left(\mathbf{x}_{t}\right) \mathrm{d} \mathbf{x}_{t}$ (in the context of Bayesian filtering, the prediction distribution serves equivalently as the priori at each filtering step, namely, $p\left(\mathbf{x}_{t}\right)$ should be specified as $\left.p\left(\mathbf{x}_{t} \mid \mathbf{z}_{1: t-1}\right)\right)$, and $\mathcal{Q}_{t}$ is the following derivative matrix

$$
\mathcal{Q}_{t}=\nabla_{\mathbf{x}_{t}^{\top}} \boldsymbol{h}\left(\mathbf{x}_{t}\right) .
$$

On the other hand, the prediction-based FIM is given by

$$
\mathcal{I}_{\mathrm{P}}^{\mathbf{x}_{t}}=-\mathbb{E}_{\mathbf{x}_{t}}\left\{\nabla_{\mathbf{x}_{t}, \mathbf{x}_{t}^{\top}} \ln p\left(\mathbf{x}_{t} \mid \mathbf{z}_{1: t-1}\right)\right\} .
$$

Considering the nonlinear and non-Gaussian properties in state transition, we employ a linearization method to cope with its nonlinear transition function $\ell\left(\mathbf{x}_{t-1}\right)$ and use a Laplace approximation [71], [79] to approximate its non-Gaussian transition distribution $p\left(\boldsymbol{\varsigma}_{t}\right)$ as follows

$$
\begin{aligned}
\mathcal{P}_{t} & =\nabla_{\mathbf{x}_{t-1}^{\top}} \ell\left(\mathbf{x}_{t-1}\right), \\
p\left(\boldsymbol{\varsigma}_{t}\right) & \approx \mathcal{N}\left(\boldsymbol{\varsigma}_{t} \mid \mathbf{0}, \boldsymbol{\chi}\right),
\end{aligned}
$$

where $\chi$ is the approximate precision matrix,

$$
\chi=\left.\nabla_{\boldsymbol{\varsigma}_{t}, \boldsymbol{\varsigma}_{t}^{\top}} \ln p\left(\boldsymbol{\varsigma}_{t}\right)\right|_{\boldsymbol{\varsigma}_{t}=\mathbf{0}} .
$$

According to the Bayesian statistics, the estimation errors asymptotically follow the Gaussian distribution as the size of measurement samples approaches infinity [26]. Hence, the previous posteriori $p\left(\mathbf{x}_{t-1} \mid \mathbf{z}_{1: t-1}\right)$ can be asymptotically approximated by a Gaussian distribution with the precision matrix $\boldsymbol{J}_{\mathrm{BE}}^{\mathbf{x}_{t-1}}$, i.e., $p\left(\mathbf{x}_{t-1} \mid \mathbf{z}_{1: t-1}\right) \approx \mathcal{N}\left(\mathbf{x}_{t-1} \mid \mathbf{x}_{t-1}^{\sharp}, \boldsymbol{J}_{\mathrm{BE}}^{\mathbf{x}_{t-1}}\right)$, where $\mathbf{x}_{t-1}^{\sharp}$ is the previous posteriori estimation [79], [80] and $\boldsymbol{J}_{\mathrm{BE}}^{\mathbf{x}_{t-1}}$ is its previous posteriori FIM. Consequently, its prediction distribution can be approximated by

$$
\begin{aligned}
& p\left(\mathbf{x}_{t} \mid \mathbf{z}_{1: t-1}\right)=\int p\left(\mathbf{x}_{t} \mid \mathbf{x}_{t-1}\right) p\left(\mathbf{x}_{t-1} \mid \mathbf{z}_{1: t-1}\right) \mathrm{d} \mathbf{x}_{t-1} \\
\approx & \int \mathcal{N}\left(\mathbf{x}_{t} \mid \mathcal{P}_{t} \mathbf{x}_{t-1}, \boldsymbol{\chi}\right) \mathcal{N}\left(\mathbf{x}_{t-1} \mid \mathbf{x}_{t-1}^{\sharp}, \boldsymbol{J}_{\mathrm{BE}}^{\mathbf{x}_{t-1}}\right) \mathrm{d} \mathbf{x}_{t-1} \\
= & \mathcal{N}\left(\mathbf{x}_{t} \mid \mathbf{x}_{t}^{p}, \boldsymbol{\Theta}_{t}^{p}\right),
\end{aligned}
$$

where the prediction expectation is $\mathbf{x}_{t}^{p}=\mathcal{P}_{t} \mathbf{x}_{t-1}^{\sharp}$ and the prediction precision is $\boldsymbol{\Theta}_{t}^{p}=\left(\mathcal{P}_{t}\left(\boldsymbol{J}_{\mathrm{BE}}^{\mathbf{x}_{t-1}}\right)^{-1} \mathcal{P}_{t}^{\top}+\chi^{-1}\right)^{-1}$.

Furthermore, its prediction-based FIM can be derived as

$$
\begin{aligned}
\mathcal{I}_{\mathrm{P}}^{\mathbf{x}_{t}} & =-\mathbb{E}_{\mathbf{x}_{t}}\left\{\nabla_{\mathbf{x}_{t}, \mathbf{x}_{t}^{\top}} \ln \mathcal{N}\left(\mathbf{x}_{t} \mid \mathbf{x}_{t}^{p}, \Theta_{t}^{p}\right)\right\} \\
& =\left\langle\left(\mathcal{P}_{t}\left(\boldsymbol{J}_{\mathrm{BE}}^{\mathbf{x}_{t-1}}\right)^{-1} \mathcal{P}_{t}^{\top}+\boldsymbol{\chi}^{-1}\right)^{-1}\right\rangle_{\mathbf{x}_{t}},
\end{aligned}
$$

which is similar to the prediction-based FIM in Eq. (71) for 
the linear and Gaussian filtering problem.

Hence, the tracking precision associated with nonlinear \& non-Gaussian filtering problem propagates as

$$
\boldsymbol{J}_{\mathrm{BE}}^{\mathbf{x}_{t}}=\left\langle\mathcal{Q}_{t}^{\top} \mathbf{W} \mathcal{Q}_{t}\right\rangle_{\mathbf{x}_{t}}+\left\langle\left(\mathcal{P}_{t}\left(\boldsymbol{J}_{\mathrm{BE}}^{\mathbf{x}_{t-1}}\right)^{-1} \mathcal{P}_{t}^{\top}+\chi^{-1}\right)^{-1}\right\rangle_{\mathbf{x}_{t}} .
$$

Remark 8. As shown in Eq. (85), the EP of the nonlinear and non-Gaussian filtering can also be depicted by the EP framework illustrated in Fig. 5. Moreover, this nonlinear \& non-Gaussian filtering problem does not account for the reference variable errors, and its EP principle complies with that of the mobile target tracking in the SLAT scheme.

The above EP analysis is based on the FIM formulation of filtering systems. Next, we reveal the propagation principle of practical filtering accuracy/error.

Due to its nonlinear and non-Gaussian nature, it is hard to find out the optimal solutions to the general nonlinear and non-Gaussian filtering problem [78]. A traditional method is to employ the linearization and Laplace approximation, such as extended Kalman filtering (EKF) algorithm [81], [82] and Laplace approximation-based filtering [71]. Note that both [81] and [78] have presented the actual filtering accuracy of the EKF algorithm, which takes a similar form as Eqs. (68)-(71), where $\mathcal{Q}_{t}$ and $\mathcal{P}_{t}$ are replaced by the linearization coefficient matrices $\dot{\mathcal{Q}}_{t}$ and $\dot{\mathcal{P}}_{t}$, respectively [78], [81], namely

$$
\begin{aligned}
& \dot{\mathcal{Q}}_{t}=\left.\nabla_{\boldsymbol{x}^{\top}} \boldsymbol{h}(\boldsymbol{x})\right|_{\boldsymbol{x}=\mathbf{x}_{t}^{p}}, \\
& \dot{\mathcal{P}}_{t}=\left.\nabla_{\boldsymbol{x}^{\top}} \boldsymbol{\ell}(\boldsymbol{x})\right|_{\boldsymbol{x}=\mathbf{x}_{t-1}^{\sharp}},
\end{aligned}
$$

where $\mathbf{x}_{t-1}^{\sharp}$ and $\mathbf{x}_{t}^{p}$ stand for its previous posteriori estimation and the prediction center, respectively.

Once again, by resorting to the inversive matrix lemma [76], its final filtering accuracy can be rewritten in a more concise form similar to Eq. (72) as

$$
\boldsymbol{\Theta}_{t}^{\sharp}=\dot{\mathcal{Q}}_{t}^{\top} \mathbf{W} \dot{\mathcal{Q}}_{t}+\left(\dot{\mathcal{P}}_{t}\left(\boldsymbol{\Theta}_{t-1}^{\sharp}\right)^{-1} \dot{\mathcal{P}}_{t}^{\top}+\chi^{-1}\right)^{-1},
$$

where $\Theta_{t}^{\sharp}$ stands for its posteriori filtering accuracy.

Obviously, its actual accuracy can also be illustrated by Fig. 5 with $\mathcal{Q}_{t}, \mathcal{P}_{t}$ replaced by $\dot{\mathcal{Q}}_{t}, \dot{\mathcal{P}}_{t}$, respectively.

3) Mobile Target Tracking: Now we turn back to the mobile target tracking EP in SLAT again to unveil its relationship with the EP behaviours of classical filtering systems.

According to the system model presented in Section III, we know that the mobile target tracking problem is equivalent to the filtering with nonlinear and non-Gaussian measurements (see Eqs. (4) and (9)). ${ }^{8}$ In particular, its measurement model incorporates a non-deterministic reference variable, i.e., there exists reference node location uncertainty $\left(\mathbf{U}_{t}^{i}\right)^{-1}$. As shown in Eq. (39), its tracking accuracy propagates in form of $\boldsymbol{J}_{\mathrm{BE}}^{\mathbf{x}_{t}}=$ $\sum_{i \in \Psi_{t}}\left(\left(\eta \mathbf{A}_{t}^{i}\right)^{-1}+\left(\mathbf{U}_{t}^{i}\right)^{-1}\right)^{-1}+\left(\left(\boldsymbol{J}_{\mathrm{BE}}^{\mathbf{x}_{t-1}}\right)^{-1}+\chi^{-1}\right)^{-1}$.

According to the CRLB derivation in Eq. (128), we know the measurement information $\eta \mathbf{A}_{t}^{i}$ can be computed as

$$
\eta \mathbf{A}_{t}^{i}=\mathbb{E}_{\mathbf{x}_{t}, \mathbf{s}_{t}^{i}, \mathrm{w}_{t}^{i}}\left\{\mathbf{g}_{t}^{i} \mathrm{w}_{t}^{i} \mathbf{g}_{t}^{i \top}\right\}
$$

\footnotetext{
${ }^{8}$ Although $\epsilon_{t}^{i}$ is assumed to be Gaussian, its precision $\mathrm{w}_{t}^{i}$ is Wishart (see Eq. (9)), it is therefore non-Gasussian distributed.
}

where $\mathbf{g}_{t}^{i}=\frac{\mathrm{d} h\left(\mathbf{x}_{t}, \mathbf{s}_{t}^{i}\right)}{\mathrm{d} \mathbf{x}_{t}}$ corresponds to the derivative of the one-dimensional measurement function $h\left(\mathbf{x}_{t}, \mathbf{s}_{t}^{i}\right)$ over $\mathbf{x}_{t}$.

Compared with the classical filtering issue addressed earlier, we know $\eta \mathbf{A}_{t}^{i}$ is equivalent to $\mathcal{Q}_{t}^{\top} \mathbf{W} \mathcal{Q}_{t}$, where $\mathcal{Q}_{t}$ is the associated gradient matrix (or linear coefficient), while $\mathbf{W}$ is the corresponding measurement precision. Hence, if we rewrite $\mathbf{g}_{t}^{i}$ and $\mathrm{w}_{t}^{i}$ in a similar form as $\mathbf{g}_{t}^{i} \Leftrightarrow \mathcal{Q}_{t}^{i \top}$ and $\mathrm{w}_{t}^{i} \Leftrightarrow \mathbf{W}_{t}^{i}$, then $\eta \mathbf{A}_{t}^{i}$ in Eq. (27) can be reformulated as

$$
\begin{aligned}
\mathbb{E}_{\mathbf{x}_{t}, \mathbf{s}_{t}^{i}, \mathrm{w}_{t}^{i}}\left\{\mathbf{g}_{t}^{i} \mathrm{w}_{t}^{i} \mathbf{g}_{t}^{i \top}\right\} & =\mathbb{E}_{\mathbf{x}_{t}, \mathbf{s}_{t}^{i}\left\{\mathcal{Q}_{t}^{i \top} \overline{\mathbf{W}}_{t}^{i} \mathcal{Q}_{t}^{i}\right\}}=\left\langle\mathcal{Q}_{t}^{i \top} \overline{\mathbf{W}}_{t}^{i} \mathcal{Q}_{t}^{i}\right\rangle_{\mathbf{x}_{t}, \mathbf{s}_{t}^{i}}
\end{aligned}
$$

where $\overline{\mathbf{W}}_{t}^{i}=\left\langle\mathbf{W}_{t}^{i}\right\rangle_{\mathbf{W}_{t}^{i}}$, and $\mathbf{W}_{t}^{i}$ is assumed to be independent of $\mathbf{x}_{t}$ and $\mathbf{s}_{t}^{i}$.

Hence, the target tracking accuracy propagates as

$$
\begin{aligned}
\boldsymbol{J}_{\mathrm{BE}}^{\mathbf{x}_{t}}= & \sum_{i \in \Psi_{t}}\left(\left(\left\langle\mathcal{Q}_{t}^{i \top} \overline{\mathbf{W}}_{t}^{i} \mathcal{Q}_{t}^{i}\right\rangle_{\mathbf{x}_{t}, \mathbf{s}_{t}^{i}}\right)^{-1}+\left(\mathbf{U}_{t}^{i}\right)^{-1}\right)^{-1} \\
& +\left(\left(\boldsymbol{J}_{\mathrm{BE}}^{\mathbf{x}_{t-1}}\right)^{-1}+\boldsymbol{\chi}^{-1}\right)^{-1}
\end{aligned}
$$

Remark 9. We can see that, the classical filtering EP principles in Eqs. (72), (85) and (88) take a similar form as the target tracking EP in Eq. (91), and the latter one is more general in the sense that it incorporates reference node location uncertainties $\left\{\left(\mathbf{U}_{t}^{i}\right)^{-1} \mid \forall i \in \Psi_{t}\right\}$, as shown in Fig. 6 .

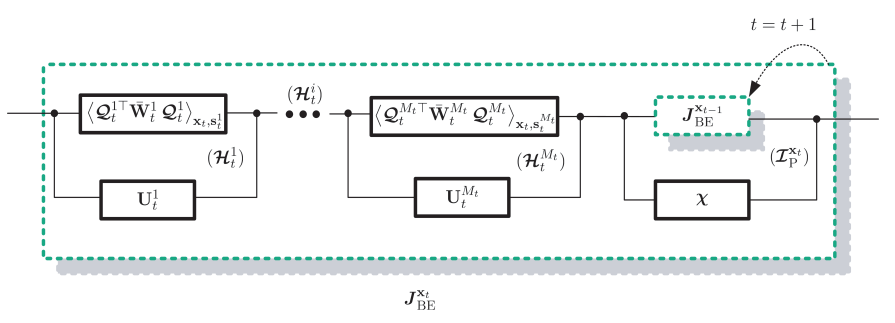

Fig. 6. An alternative illustration of the mobile target tracking EP principle.

In fact, if those $M_{t}$ independent measurements $\left\{\mathrm{z}_{t}^{i} \mid \forall i \in \Psi_{t}\right\}$ of mobile target in the SLAT scheme are processed in a joint manner, its gradient will be naturally expanded to a matrix $\mathcal{Q}_{t}=\left[\mathbf{g}_{t}^{1 \top} ; \cdots ; \mathbf{g}_{t}^{M_{t} \top}\right]$, then the measurement information of the SLAT scheme can be reformulated by a joint-form $\left\langle\mathcal{Q}_{t}^{\top} \overline{\mathbf{W}}_{t} \mathcal{Q}_{t}\right\rangle_{\mathbf{x}_{t}, \mathbf{s}_{t}}$, if we neglect the reference uncertainties, where $\overline{\mathbf{W}}_{t}=\operatorname{diag}\left[\left\langle\mathrm{w}_{t}^{1}\right\rangle_{\mathrm{w}_{t}^{1}}, \cdots,\left\langle\mathrm{w}_{t}^{M_{t}}\right\rangle_{\mathrm{w}_{t}^{M_{t}}}\right]$. Here, $\operatorname{diag}[\cdots]$ denotes a diagonal matrix.

In this way, the SLAT-associated measurement information can be rewritten in a similar form as those in classical filtering problems (see Eqs. (72), (85) and (88)).

Generally speaking, the joint-form measurement information $\left\langle\mathcal{Q}_{t}^{\top} \overline{\mathbf{W}}_{t} \mathcal{Q}_{t}\right\rangle_{\mathbf{x}_{t}, \mathbf{s}_{t}}$ can incorporate the correlation among different measurements; while if measurements are mutually independent, the joint-form information is equivalent to series connection (summation) of those independent individual information, i.e., $\left\langle\mathcal{Q}_{t}^{\top} \overline{\mathbf{W}}_{t} \mathcal{Q}_{t}\right\rangle_{\mathbf{x}_{t}, \mathbf{s}_{t}}=\sum_{i \in \Psi_{t}}\left\langle\mathbf{g}_{t}^{i} \overline{\mathbf{w}}_{t}^{i} \mathbf{g}_{t}^{i \top}\right\rangle_{\mathbf{x}_{t}, \mathbf{s}_{t}^{i}}$.

The above analysis provides us a general framework to evaluate the performance and error propagation principle of the general nonlinear \& non-Gaussian filtering problem, particularly in the presence of non-deterministic reference variables, 
which coincides with that of the SLAT issue.

\section{Convergence Analysis}

In this section, we examine the convergence conditions, convergence properties and robustness of mobile tracking and node location calibration EP principles of the SLAT scheme.

\section{A. Convergence Conditions}

The convergence conditions guarantee that the mobile target tracking and node calibration are effective with decreased errors, which is important for practical algorithm design. First, we focus on the EP convergence for mobile target tracking.

The random transition of mobile target location can render a lower prediction precision $\mathcal{I}_{\mathrm{P}}^{\mathbf{x}_{t}}$ than its latest posteriori $\boldsymbol{J}_{\mathrm{BE}}^{\mathbf{x}_{t-1}}$. That is, there is information loss in the location prediction stage, due to the random transition of mobile target.

Definition 1. The information loss in the location prediction stage is defined as

$$
\begin{aligned}
\mathcal{L}_{t} & =\boldsymbol{J}_{\mathrm{BE}}^{\mathbf{x}_{t-1}}-\mathcal{I}_{\mathrm{P}}^{\mathbf{x}_{t}} \\
& =\left(\left(\mathcal{B}_{\mathrm{BE}}^{\mathbf{x}_{t-1}}\right)^{\top} \boldsymbol{\chi} \mathcal{B}_{\mathrm{BE}}^{\mathbf{x}_{t-1}}+\mathcal{B}_{\mathrm{BE}}^{\mathbf{x}_{t-1}}\right)^{-1} .
\end{aligned}
$$

Theorem 1. If the overall equivalent measurement information $\mathcal{H}_{t}$ can compensate the localization information loss $\mathcal{L}_{t}$ due to target's random mobility, i.e.,

$$
\mathcal{H}_{t} \succ \mathcal{L}_{t}
$$

the target tracking error tends to diminish over time, i.e., $\boldsymbol{J}_{\mathrm{BE}}^{\mathbf{x}_{t}} \prec \boldsymbol{J}_{\mathrm{BE}}^{\mathbf{x}_{t-1}}, \forall t=1,2, \cdots$ where $\mathcal{H}_{t}$ is given in Eqs. (39) and (41), and $\succ$ means $A-B$ is positive if the squared matrices $\mathbf{A}$ and $B$ satisfy $A \succ B$.

Proof: Refer to APPENDIX F.

Remark 10. The overall equivalent measurement information $\mathcal{H}_{t}$ should be able to compensate for the information loss $\mathcal{L}_{t}$, i.e., $\mathcal{H}_{t} \succ \mathcal{L}_{t}$, to guarantee a converged target tracking error.

According to Eq. (92), $\mathcal{L}_{t}$ depends on the latest tracking error $\mathcal{B}_{\mathrm{BE}}^{\mathbf{x}_{t-1}}$ and the location transition precision $\chi$. It means that, within the current time window, $\mathcal{L}_{t}$ is determined by the own state of mobile target, while it is independent of the external measurement state. On the contrary, from Eq. (39) we know $\mathcal{H}_{t}$ is completely dependent on the external measurement state associated with reference sensor nodes. More specifically, it is dependent on the number of reference nodes, the reference node location's prior precision $\mathbf{U}_{t}^{i}$ and the associated resolution factor $\mathbf{A}_{t}^{i}$. Hence, in order to guarantee a converged tracking error, proper reference node selection [21], sensor node deployment [48] or measurement strategy design based on Theorem 1 is preferred.

Next, we study the convergence condition for the reference sensor node location calibration.

Theorem 2. If the measurement precision factor $\eta>0$ and at least one of reference sensor node location precisions satisfies $\mathbf{U}_{t}^{j} \succ \mathbf{0}, j \in \Phi_{t}^{i}$, or the mobile target tracking accuracy satisfies $\mathcal{I}_{\mathrm{BE}}^{\mathrm{x}_{t}} \succ \mathbf{0}$, then the location calibration error propagation of the objective sensor node $\mathbf{s}_{t}^{i}, \forall i \in \Psi_{t}$, converges, i.e., $\mathcal{I}_{\mathrm{BE}}^{\mathrm{s}_{t}^{i}} \succ \mathbf{U}_{t}^{i}$.
Proof: Refer to the proof in APPENDIX G.

We know the measurement precision factor $\eta$ is just the expectation $\Omega \psi$ of random measurement precision $\mathrm{w}_{t}^{i}$ and $\mathrm{w}_{t}^{i, j}$ (see Eq. (34)), therefore, $\eta>0$ is equivalent to $\Omega \psi>0$, which means the reference node's measurement is effective.

Remark 11. Only when the location error of at least one reference node (including the mobile target node) is finite and its measurement data is effective, the objective sensor node location calibration error converges. Hence, through sufficient calibration procedures in the SLAT scheme, the locations of those inaccurate sensor nodes will gradually become accurate.

\section{B. Balance State}

The balance state of tracking EP is examined next to gain insights into the inherent mechanism of SLAT scheme.

Theorem 3. The localization information loss $\mathcal{L}_{t}$ in target location prediction stage increases with the growth of the latest tracking accuracy $J_{\mathrm{BE}}^{\mathrm{x}_{t-1}}$. In addiion, $\mathcal{L}_{t}$ follows that

$$
\begin{aligned}
\lim _{\mathcal{B}_{\mathrm{BE}}^{\mathbf{x}_{t-1} \rightarrow \mathbf{0}}} \mathcal{L}_{t}=\boldsymbol{\infty} \\
\lim _{\mathcal{B}_{\mathrm{BE}}^{\mathbf{x}_{t-1} \rightarrow \infty}} \mathcal{L}_{t}=\mathbf{0} .
\end{aligned}
$$

Proof: See the proof in APPENDIX H.

Remark 12. We can see from Theorem 3 that, the mobile target with higher tracking accuracy at the latest round will lose more localization information in the location prediction stage, due to its random transition. When the latest tracking accuracy is sufficient large, the localization information loss will become infinite.

At the beginning of SLAT, the localization information loss $\mathcal{L}_{t}$ is very small due to limited priori information, the measurements can easily compensate for the information loss $\mathcal{L}_{t}$, resulting in a quick convergence behaviour of the target tracking EP, in the beginning of SLAT.

As the SLAT scheme proceeds, the posteriori information $J_{\mathrm{BE}}^{\mathbf{x}_{t-1}}$ becomes larger and larger, so does the information loss $\mathcal{L}_{t}$, which implies a slower convergence rate of the tracking accuracy $\boldsymbol{J}_{\mathrm{BE}}^{\mathrm{X}_{t}}$ in the following time instants. When $\mathcal{L}_{t}$ becomes slightly larger than $\mathcal{H}_{t}$ due to the continuous increase in $\boldsymbol{J}_{\mathrm{BE}}^{\mathrm{x}_{t-1}}$, i.e., the measurements can not compensate for the information loss, the current tracking precision $\boldsymbol{J}_{\mathrm{BE}}^{\mathbf{x}_{t}}$ will become slightly lower than its previous value $\boldsymbol{J}_{\mathrm{BE}}^{\mathbf{x}_{t-1}}$. This decrease in the tracking information $\boldsymbol{J}_{\mathrm{BE}}^{\mathbf{x}_{t-1}}$ leads to reduced $\mathcal{L}_{t}$. Therefore, at the next time instant, the overall equivalent measurement information $\mathcal{H}_{t}$ is able to compensate for the information loss $\mathcal{L}_{t}$ again.

Consequently, the mobile tracking precision increases over time when the measurement information could compensate for the information loss in location prediction stage.

As such, the interaction between dynamic $\mathcal{H}_{t}$ and $\mathcal{L}_{t}$ will finally achieve a balance state of tracking information, wherein a stationary tracking error performance holds, as illustrated in Fig. 7. In other words, there will be a balance state $\mathcal{B}_{\mathrm{BE}}^{\mathrm{x}}$ of the target tracking EP, where $\mathcal{H}_{t}=\mathcal{L}_{t}\left(\mathcal{B}_{\mathrm{BE}}^{\mathrm{x} \star}\right)$, i.e.,

$$
\left(\mathcal{B}_{\mathrm{BE}}^{\mathrm{x} \star}\right)^{\top} \chi \mathcal{B}_{\mathrm{BE}}^{\mathrm{x} \star}+\mathcal{B}_{\mathrm{BE}}^{\mathrm{x} \star}-\mathcal{H}_{t}^{-1}=\mathbf{0},
$$


which falls into the framework of algebraic Reccati equation (ARE) [83]. The above ARE equation can be applied to any nonlinear \& non-Gaussian filtering problem as well.

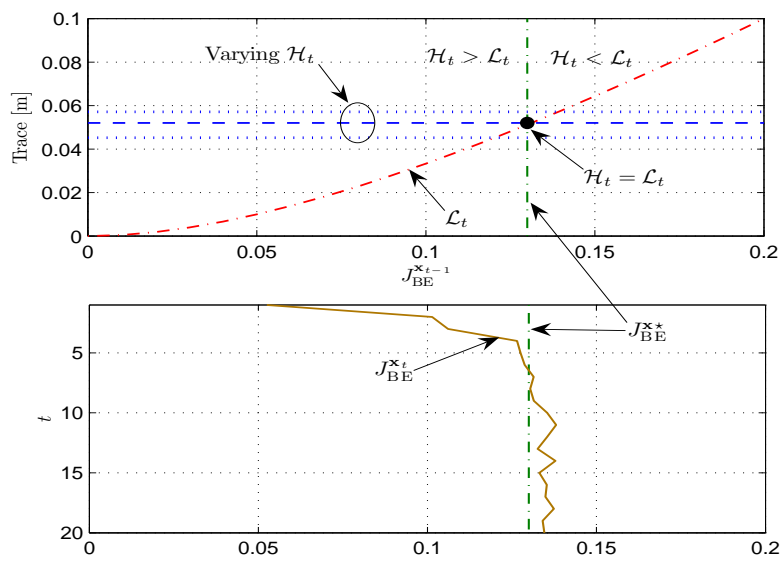

Fig. 7. The mechanism of tracking EP convergence. The upper subfigure shows $\mathcal{H}_{t}$ versus $\mathcal{L}_{t}$ over $\boldsymbol{J}_{\mathrm{BE}}^{\mathbf{x}_{t-1}}$ where their intersection is the balance state $\boldsymbol{J}_{\mathrm{BE}}^{\mathbf{x} \star}$. The lower subfigure shows the converging curve of $\boldsymbol{J}_{\mathrm{BE}}^{\mathbf{x}_{t}}$ over time, which complies with the mechanism unveiled in the upper subfigure.

Theorem 4. In a sufficient long-time scale, given measurement set $\left\{\mathrm{z}_{\tau}^{i} \mid \forall i \in \Psi_{\tau}, \forall \tau=1: t\right\}$ from inaccurate reference nodes $\left\{\boldsymbol{\mu}_{\tau}^{i}, \mathbf{U}_{\tau}^{i} \mid \forall i \in \Psi_{\tau}, \forall \tau=1: t\right\}$, the target tracking error will converge to a balance state, i.e., the solution to balance function in Eq. (96), which is expressed as

$$
\mathcal{B}_{\mathrm{BE}}^{\mathrm{x} \star}=\frac{1}{2} \chi^{-\frac{1}{2}}\left(\mathbf{I}+4 \chi^{\frac{1}{2}} \mathcal{H}_{t}^{-1} \chi^{\frac{1}{2}}\right)^{\frac{1}{2}} \chi^{-\frac{1}{2}}-\frac{1}{2} \chi^{-1},
$$

where the overall measurement information $\mathcal{H}_{t}$ (see Eqs. (39) and (41)) and the location transition precision matrix $\chi$ are assumed to be positive semidefinite.

Proof: See the proof of Eq. (97) in APPENDIX I.

The balance state of tracking information propagation is defined accordingly as $\boldsymbol{J}_{\mathrm{BE}}^{\mathbf{x} \star}=\left(\boldsymbol{B}_{\mathrm{BE}}^{\mathbf{x}}\right)^{-1}$.

Remark 13. From Eq. (97) we know that, the balance state $\mathcal{B}_{\mathrm{BE}}^{\mathrm{x} \star}$ of tracking error is completely determined by the location transition precision (the own state of mobile target) and the overall equivalent measurement information $\mathcal{H}_{t}$ (the external state associated with reference nodes). Hence, performing an efficient reference node selection scheme or sensor node placement based on Theorem $\mathbf{4}$ is important in order to realize a lower tracking error balance state $\mathcal{B}_{\mathrm{BE}}^{\mathrm{x} \star}$ with a minimum number of eminent reference nodes.

On the other hand, given the equivalent measurement information intensity $\mathcal{H}_{t}$ and target transition precision $\chi$, there are upper and lower bounds for the final target tracking EP.

Theorem 5. The balance state $\mathcal{B}_{\mathrm{BE}}^{\mathrm{x}}$ of tracking error is bounded by

$$
\Psi_{t}^{\prime} \preceq \mathcal{B}_{\mathrm{BE}}^{\mathrm{x} \star} \preceq \Psi_{t}^{\prime \prime},
$$

where the lower and upper bounds are defined as

$$
\begin{aligned}
\boldsymbol{\Psi}_{t}^{\prime} & =\left(\mathcal{H}_{t}+\boldsymbol{\chi}\right)^{-1}, \\
\boldsymbol{\Psi}_{t}^{\prime \prime} & =\mathcal{H}_{t}^{-1} .
\end{aligned}
$$

Proof: Please see the proof in APPENDIX J.

\section{Convergence Characteristics}

We focus on the convergence properties of tracking EP, e.g., the convergence rate, fluctuation degree of the balance state and its balance state $\boldsymbol{J}_{\mathrm{BE}}^{\mathbf{x}}$.

Equivalent measurement information $\mathcal{H}_{t}$ and transition precision $\chi$ jointly determine how fast $\boldsymbol{J}_{\mathrm{BE}}^{\mathrm{x}_{t}}$ converges over time.

Definition 2. Let us define the information improvement as

$$
\begin{aligned}
\boldsymbol{\rho}_{t} & =\mathcal{H}_{t}-\mathcal{L}_{t} \\
& =\mathcal{H}_{t}-\left(\left(\mathcal{B}_{\mathrm{BE}}^{\mathbf{x}_{t-1}}\right)^{\top} \chi \mathcal{B}_{\mathrm{BE}}^{\mathbf{x}_{t-1}}+\mathcal{B}_{\mathrm{BE}}^{\mathbf{x}_{t-1}}\right)^{-1},
\end{aligned}
$$

which can also indicate the EP convergence rate.

We can see that, larger values of $\mathcal{H}_{t}$ and $\chi$ result in larger $\boldsymbol{\rho}_{t}$, which corresponds to faster convergence of tracking EP. Based on Eq. (97) we may further conclude that larger $\mathcal{H}_{t}$ leads to lower balance error $\mathcal{B}_{\mathrm{BE}}^{\mathrm{x}}$.

Due to the randomness in $\mathcal{H}_{t}$ resulted from the changing reference nodes and target movement, the balance state $\boldsymbol{J}_{\mathrm{BE}}^{\mathbf{x} \star}$ of tracking accuracy fluctuates over time. Let $\mathbf{H}_{t}$ denote the degree of fluctuation of balance accuracy $\boldsymbol{J}_{\mathrm{BE}}^{\mathrm{x} \star}$, which is defined as the covariance matrix of the squared matrix $\boldsymbol{J}_{\mathrm{BE}}^{\mathrm{x} \star}$, namely, $\mathbf{H}_{t}=\operatorname{cov}\left(\boldsymbol{J}_{\mathrm{BE}}^{\mathbf{x}}\right)$.

The randomness of $\mathcal{H}_{t}$ implies how intense the associated balance state $\boldsymbol{J}_{\mathrm{BE}}^{\mathrm{x} \star}$ fluctuates. Assume the precision matrix of random $\mathcal{H}_{t}$ is $\mathbf{V}$.

Theorem 6. Given the precision matrix $\mathrm{V}$ of equivalent measurement information $\mathcal{H}_{t}$, the fluctuation degree of balance state $J_{\mathrm{BE}}^{\mathrm{x} \star}$ can be approximated by

$$
\widehat{\mathbf{H}}_{t} \approx\left(\left\langle\mathbf{G}_{t}^{\top} \mathbf{V} \mathbf{G}_{t}\right\rangle_{\mathbf{x}_{t}, \mathbf{s}_{t}}\right)^{-1},
$$

where the matrix $\mathrm{G}_{t}$ is approximated by

$$
\mathbf{G}_{t}=\left(\mathcal{B}_{\mathrm{BE}}^{\mathbf{x} \star \top} \chi+\mathbf{I}\right)^{-1}\left(\chi^{\top} \mathcal{B}_{\mathrm{BE}}^{\mathbf{x} \star}+\mathcal{B}_{\mathrm{BE}}^{\mathbf{x} \star \top} \chi+\mathbf{I}\right)\left(\chi^{\top} \mathcal{B}_{\mathrm{BE}}^{\mathbf{x} \star}+\mathbf{I}\right)^{-1} .
$$

Proof: See the proof of Theorem 6 in APPENDIX K.

In addition, we know larger measurement information $\mathcal{H}_{t}$ leads to smaller tracking errors $\mathcal{B}_{\mathrm{BE}}^{\mathrm{x} \star}$ (see Eq. (97)), and renders an increase in $\mathbf{G}_{t}$ (see Eq. (103)), which will in return result in smaller fluctuation degree $\mathbf{H}_{t}$. Therefore, the balanced state $\mathcal{B}_{\mathrm{BE}}^{\mathrm{x} \star}$ is more stable with larger $\mathcal{H}_{t}$.

In summary, as analysed above, larger measurement information $\mathcal{H}_{t}$ is highly desirable in terms of lower tracking error, faster convergence rate $\rho_{t}$ (see Eq. (101)) and more stable balance state, which complies with our common sense.

\section{Robustness of Tracking EP}

In this part, the robustness of SLAT scheme is demonstrated.

Suppose there is a sudden tracking failure at a certain time instant, which leads to a large posterior error $\mathcal{B}_{\mathrm{BE}}^{\mathbf{x}_{t-1}}$. This situation is similar to the initial stage of the SLAT scheme when there is very little information $\boldsymbol{J}_{\mathrm{BE}}^{\mathbf{x}_{t-1}}$, which leads to very little information loss $\mathcal{L}_{t}$.

As unveiled in Eq. (95) (in the proof of Theorem 3 in APPENDIX H), due to limited prediction information loss $\mathcal{L}_{t}$, 
at the next time instant, the measurements can easily and immediately compensate for the information loss, and the SLAT scheme can achieve the EP balance state again.

\section{Asymptotic CRLB AnAlysis}

In this section, the asymptotic performance limits of mobile tracking and node location calibration EP principles of the SLAT scheme are demonstrated, which can provide guidelines for the practical network design to strike a balance between the performance benefits and costs.

\section{A. Impact of Random Measurement Precision}

Based on Eqs. (39) and (63), we know that both the target tracking accuracy and the sensor location calibration accuracy depend on the expectation of measurement precision, in the presence of random measurements.

We know measurement precision factor $\eta$ is also the associated expectation $\psi \Omega$. At this point, the CRLB on the target tracking of the SLAT scheme follows that

$$
\begin{aligned}
\lim _{\eta \rightarrow \infty} \mathcal{B}_{\mathrm{BE}}^{\mathbf{x}_{t}} & =\left(\sum_{i \in \Psi_{t}} \mathbf{U}_{i}+\left(\left(\boldsymbol{J}_{\mathrm{BE}}^{\mathbf{x}_{t-1}}\right)^{-1}+\chi^{-1}\right)^{-1}\right)^{-1}, \\
\lim _{\eta \rightarrow 0} \mathcal{B}_{\mathrm{BE}}^{\mathbf{x}_{t}} & =\left(\boldsymbol{J}_{\mathrm{BE}}^{\mathbf{x}_{t-1}}\right)^{-1}+\boldsymbol{\chi}^{-1} .
\end{aligned}
$$

Remark 14. This indicates that, (i) when the observations are definitely accurate (or the measurement size is infinitely large), the equivalent measurement information $\mathcal{H}_{t}^{i}$ is upgraded to the reference node location precision $\mathbf{U}_{t}^{i}$, i.e., there is no localization information loss due to environment distortions; (ii) if the observation completely fails, the final tracking information is just the prediction information.

On the other hand, the CRLB associated with sensor node location calibration follows that

$$
\begin{aligned}
\lim _{\eta \rightarrow \infty} \mathcal{B}_{\mathrm{BE}}^{\mathbf{s}_{t}^{i}} & =\left(\sum_{j \in \Phi_{t}^{i}} \mathbf{U}_{t}^{j}+\mathbf{U}_{t}^{i}+\sum_{k \in \Psi_{t}} \mathbf{U}_{t}^{k}+\mathcal{I}_{\mathrm{P}}^{\mathbf{x}_{t}}\right)^{-1}, \\
\lim _{\eta \rightarrow 0} \mathcal{B}_{\mathrm{BE}}^{\mathbf{s}_{t}^{i}} & =\left(\mathbf{U}_{t}^{i}\right)^{-1} .
\end{aligned}
$$

We can reach the same conclusions for calibration as those for mobile tracking in Remark 14.

\section{B. Impact of Sensor Node Location Errors}

We examine the asymptotic limits of mobile tracking over reference node location errors at first. Then, the asymptotic limits of sensor node location calibration will be presented.

Considering the sensor node location uncertainties, the target tracking accuracy at current time instant can be calculated asymptotically as

$$
\begin{aligned}
\lim _{\substack{i \rightarrow \infty \\
\mathbf{U}_{t}^{i} \rightarrow \Psi_{t},}} \boldsymbol{J}_{\mathrm{BE}}^{\mathbf{x}_{t}} & =\underbrace{\sum_{\substack{\mathbf{U}_{t}^{i} \rightarrow \mathbf{0}, \forall i \in \Psi_{t}}} \boldsymbol{J}_{\mathrm{BE}}^{\mathbf{x}_{t}}=\widetilde{\mathbf{A}}_{t}^{i}+\mathcal{I}_{\mathrm{P}}^{\mathbf{x}_{t}},}_{\boldsymbol{J}_{\infty}^{\mathbf{x}_{t}}},
\end{aligned}
$$

where

$$
\widetilde{\mathbf{A}}_{t}^{i}=\mathbb{E}_{\mathbf{x}_{t}}\left\{\frac{\left(\mathbf{x}_{t}-\boldsymbol{\mu}_{t}^{i}\right)\left(\mathbf{x}_{t}-\boldsymbol{\mu}_{t}^{i}\right)^{\top}}{\left\|\mathbf{x}_{t}-\boldsymbol{\mu}_{t}^{i}\right\|_{2}^{4}}\right\}
$$

and $\mathbf{U}_{t}^{i} \rightarrow \infty$ stands for $\left\|\mathbf{U}_{t}^{i}\right\|_{\mathrm{H}}>\|\mathbf{M}\|_{\mathrm{H}}, \forall \mathbf{M} \in \mathbb{R}^{D \times D}$, and $\|\bullet\|_{\mathrm{H}}$ denotes the associated Hilbert-Schmidt norm.

Theorem 7. Considering reference node location errors, the tracking accuracy $J_{\mathrm{BE}}^{\mathrm{x}_{t}}$ is bounded by

$$
\boldsymbol{J}_{\infty}^{\mathbf{x}_{t}} \preceq \boldsymbol{J}_{\mathrm{BE}}^{\mathbf{x}_{t}} \preceq \mathcal{I}_{\mathrm{P}}^{\mathbf{x}_{t}},
$$

where $J_{\infty}^{\mathrm{x}_{t}}$ is given by Eq. (108).

Proof: This theorem can be directly proved by the fact that, $\boldsymbol{J}_{\mathrm{BE}}^{\mathbf{x}_{t}}$ is non-decreasing with the precision $\mathbf{U}_{t}^{i}$ of its reference sensor location, $\forall i \in \Psi_{t}$, based on Eq. (39).

If all reference node locations are completely inaccurate during entire SLAT stage, the target tracking fails, i.e.,

$$
\lim _{\substack{\mathbf{U}_{\tau}^{i} \rightarrow \mathbf{0}, \forall i \in \Psi_{\tau}, \forall \tau=1: t}} \boldsymbol{J}_{\mathrm{BE}}^{\mathbf{x}_{t}}=\lim _{\substack{\mathbf{U}_{\tau}^{i} \rightarrow \mathbf{0}, \forall i \in \mathbf{0}_{\tau}, \forall \tau=1: t}} \mathcal{I}_{\mathrm{P}}^{\mathbf{x}_{t}}=\mathbf{0} .
$$

Let us evaluate the sensor node location calibration performance over the reference node location errors.

If the location of each reference node of the objective node $\mathbf{s}_{t}^{i}$ is accurate, then the calibration accuracy asymptotically approaches $\boldsymbol{J}_{\infty}^{\mathbf{s}_{t}^{i}}$ as shown in Eq. (113) where

$$
\widetilde{\mathbf{A}}_{t}^{i, j}=\mathbb{E}_{\mathbf{s}_{t}^{i}}\left\{\frac{\left(\mathbf{s}_{t}^{i}-\boldsymbol{\mu}_{t}^{j}\right)\left(\mathbf{s}_{t}^{i}-\boldsymbol{\mu}_{t}^{j}\right)^{\top}}{\left\|\mathbf{s}_{t}^{i}-\boldsymbol{\mu}_{t}^{j}\right\|_{2}^{4}}\right\} .
$$

Obviously, if the objective node location is also accurate, the calibration precision is infinitely large, i.e.,

$$
\lim _{\mathbf{U}_{t}^{i} \rightarrow \infty} \boldsymbol{J}_{\infty}^{\mathbf{s}_{t}^{i}}=\infty
$$

On the other hand, when the locations of those reference nodes of the objective node $\mathbf{s}_{t}^{i}$ are not so accurate, the calibration precision will be

$$
\begin{aligned}
\lim _{\substack{\mathbf{U}_{t}^{k}, \mathbf{U}_{t}^{j} \rightarrow \mathbf{0}, \forall j \in \Phi_{i}^{i}, \forall k \in \Psi_{t}, k \neq i}} \boldsymbol{J}_{\mathrm{BE}}^{\mathbf{s}_{t}^{i}} & =\left(\left(\eta \mathbf{A}_{t}^{i}\right)^{-1}+\left(\lim _{\substack{\mathbf{U}_{t}^{i} \rightarrow \mathbf{0}, \forall k \in \Psi_{t}, k \neq i}} \boldsymbol{J}_{\mathrm{BE}}^{\mathbf{x}_{t}}\right)^{-1}\right)^{-1}+\mathbf{U}_{t}^{i} \\
& =\underbrace{\left(2\left(\eta \mathbf{A}_{t}^{i}\right)^{-1}+\left(\mathbf{U}_{t}^{i}\right)^{-1}\right)^{-1}+\mathbf{U}_{t}^{i}}_{\boldsymbol{J}_{0}^{\mathbf{s}_{t}^{i}}}
\end{aligned}
$$

Let $\mathcal{B}_{\mathbf{0}}^{\mathbf{s}_{t}^{i}}=\left(\boldsymbol{J}_{\mathbf{0}}^{\mathbf{s}_{t}^{i}}\right)^{-1}$. If there is no priori information for the objective node $\mathbf{s}_{t}^{i}$, the sensor calibration will fail, i.e.,

$$
\lim _{\mathbf{U}_{t}^{i} \rightarrow \mathbf{0}} \boldsymbol{J}_{\mathbf{0}}^{\mathbf{s}_{t}^{i}}=\mathbf{0} .
$$

Theorem 8. Considering reference node location uncertainties, the calibration accuracy $J_{\mathrm{BE}}^{\mathrm{s}_{t}^{2}}$ is bounded by

$$
\boldsymbol{J}_{\infty}^{\mathbf{s}_{t}^{i}} \preceq \boldsymbol{J}_{\mathrm{BE}}^{\mathbf{s}_{t}^{i}} \preceq \boldsymbol{J}_{\mathbf{0}}^{\mathbf{s}_{t}^{i}},
$$

where $J_{\infty}^{\mathrm{s}_{t}^{i}}$ and $J_{0}^{\mathrm{s}_{t}^{i}}$ are given by Eqs. (113) and (116), respectively.

Proof: This conclusion can be directly inferred from the 


$$
\begin{aligned}
\lim _{\substack{\mathbf{U}_{t}^{k}, \mathbf{U}_{t}^{j} \rightarrow \infty, \forall j \in \Phi_{t}^{i}, \forall k \in \Psi_{t}, k \neq i}} \boldsymbol{J}_{\mathrm{BE}}^{\mathbf{s}_{t}^{i}} & =\left(\left(\lim _{\substack{\mathbf{U}_{t}^{k} \rightarrow \infty, \forall k \in \Psi_{t}, k \neq i}} \boldsymbol{J}_{\mathrm{BE}}^{\mathbf{x}_{t}}\right)^{-1}+\left(\eta \widetilde{\mathbf{A}}_{t}^{i}\right)^{-1}\right)^{-1}+\sum_{j \in \Phi_{t}^{i}} \eta \widetilde{\mathbf{A}}_{t}^{i, j}+\mathbf{U}_{t}^{i} \\
& =\underbrace{\left(\left(\sum_{k \in \Psi_{t}, k \neq i} \eta \widetilde{\mathbf{A}}_{t}^{k}+\mathcal{I}_{\mathrm{P}}^{\mathbf{x}_{t}}+\left(\left(\eta \widetilde{\mathbf{A}}_{t}^{i}\right)^{-1}+\left(\mathbf{U}_{t}^{i}\right)^{-1}\right)^{-1}\right)^{-1}+\left(\eta \widetilde{\mathbf{A}}_{t}^{i}\right)^{-1}\right)^{-1}+\sum_{j \in \Phi_{t}^{i}} \eta \widetilde{\mathbf{A}}_{t}^{i, j}+\mathbf{U}_{t}^{i}}_{\mathbf{J}_{\infty}^{\mathbf{s}_{t}^{i}}} .
\end{aligned}
$$

fact that, $\boldsymbol{J}_{\mathrm{BE}}^{\mathbf{s}_{t}^{i}}$ is non-decreasing with the precision $\mathbf{U}_{t}^{j}$ of its reference sensor location, $\forall j \in \Phi_{t}^{i}$, based on Eq. (63).

\section{Impact of Sensor Node Density}

In the following, we quantify the relationship between the tracking/calibration accuracy and the sensor node density.

Theorem 9. When all sensor nodes are uniformly deployed inside the coverage area, and their precisions are independently and identically distributed, both tracking accuracy and calibration accuracy scale linearly with their reference cluster sizes, i.e.,

$$
\begin{aligned}
& \overline{\boldsymbol{J}}_{\mathrm{BE}}^{\mathbf{x}_{t}}=M_{t} \overline{\mathcal{H}}_{t}+\mathcal{\mathcal { I }}_{\mathrm{P}}^{\mathbf{x}_{t}}, \\
& \overline{\boldsymbol{J}}_{\mathrm{BE}}^{\mathbf{s}_{t}^{i}}=N_{t} \overline{\mathcal{H}}_{t}+\overline{\mathcal{H}}_{t}^{\mathbf{x}}+\mathbf{U}_{t}^{i},
\end{aligned}
$$

where the equivalent measurement information expectation $\overline{\mathcal{H}}_{t}$ and $\overline{\mathcal{H}}_{t}^{\mathrm{x}}$ are given, respectively, by

$$
\begin{aligned}
\overline{\mathcal{H}}_{t} & =\left(\left(\eta \boldsymbol{\Lambda}_{t}\right)^{-1}+\overline{\mathbf{U}}_{t}^{-1}\right)^{-1}, \\
\overline{\mathcal{H}}_{t}^{\mathbf{x}} & =\left(\left(\eta \boldsymbol{\Lambda}_{t}\right)^{-1}+\left(\mathcal{I}_{\mathrm{BE}}^{\mathbf{x}_{t}}\right)^{-1}\right)^{-1} .
\end{aligned}
$$

In addition, $\overline{\mathbf{U}}_{t}$ means the averaged precision of inaccurate sensor node locations, which is given in Eq. (157), and $\Lambda_{t}$ denotes the averaged geometric resolution, which is given in Eq. (156).

Proof: See the proof in APPENDIX L.

Remark 15. We can see that, even if all node locations are inaccurate, the tracking and calibrating error can be arbitrarily small, if the reference cluster size is sufficiently large, with no special assumptions for all reference sensors.

\section{Asymptotic Limits of Balance State $\mathcal{B}_{\mathrm{BE}}^{\mathrm{x} \star}$}

The balance state $\mathcal{B}_{\mathrm{BE}}^{\mathrm{x} \star}$ reflects the final target tracking performance, which is also dependent of sensor node location uncertainties. The following theorem shows the impact of the sensor node location uncertainties on the mobility tracking performance.

Theorem 10. Considering the sensor node location uncertainties, the asymptotic performance of the mobile tracking error is derived as

$$
\lim _{\substack{\mathbf{U}_{t}^{i} \rightarrow \infty, \forall i \in \Psi_{t}}} \mathcal{B}_{\mathrm{BE}}^{\mathrm{X} \star}=\underbrace{\frac{1}{2} \boldsymbol{\chi}^{-\frac{1}{2}}\left(\mathbf{I}+4 \boldsymbol{\chi}^{\frac{1}{2}} \widetilde{\mathcal{H}}_{t}^{-1} \chi^{\frac{1}{2}}\right)^{\frac{1}{2}} \chi^{-\frac{1}{2}}-\frac{1}{2} \chi^{-1}}_{\mathcal{B}_{\infty}^{\mathrm{x}}},
$$

where $\widetilde{\mathcal{H}}_{t}=\sum_{i \in \Psi_{t}} \eta \widetilde{\mathbf{A}}_{t}^{i}$ and $\widetilde{\mathbf{A}}_{t}^{i}$ is given in Eq. (110).
Proof: Eq. (123) can be obtained straightforwardly based on Eq. (97).

Remark 16. The asymptotic limit $\mathcal{B}_{\infty}^{\mathrm{x} \star}$ serves as the the lower bound of tracking error balance state $\mathcal{B}_{\mathrm{BE}}^{\mathrm{x} \star}$ when the reference sensor node location is completely accurate.

In addition, regarding the influence of random measurement precision, we have a conclusion below.

Theorem 11. Considering the measurement precision, the balance state of tracking error follows that,

$$
\begin{aligned}
\lim _{\eta \rightarrow \infty} \mathcal{B}_{\mathrm{BE}}^{\mathrm{x} \star}= & \frac{1}{2} \boldsymbol{\chi}^{-\frac{1}{2}}\left(\mathbf{I}+4 \boldsymbol{\chi}^{\frac{1}{2}}\left(\sum_{i \in \Psi_{t}} \mathbf{U}_{t}^{i}\right)^{-1} \chi^{\frac{1}{2}}\right)^{\frac{1}{2}} \chi^{-\frac{1}{2}} \\
& -\frac{1}{2} \boldsymbol{\chi}^{-1} \\
\lim _{\eta \rightarrow 0} \mathcal{B}_{\mathrm{BE}}^{\mathrm{x} \star}= & \infty
\end{aligned}
$$

Proof: Eqs. (124) and (125) can be directly derived based on Eq. (97).

Remark 17. Eqs. (124) and (125) present the lower and upper bounds of tracking error balance state $\mathcal{B}_{\mathrm{BE}}^{\mathrm{x} \star}$, respectively, when the measurement data is definitely accurate and not effective at all. The conclusions are identical to those in Remark 14.

\section{NumERICAL RESUlTS}

In this section, we present extensive simulation results to corroborate all the asymptotic performance limit and EP analysis. Four different scenarios A1-A4 and associated parameter settings are summarized in TABLE II.

\section{A. Simulation Settings}

In order to study the effect of target mobility and random measurement precision on the achieved SLAT performance, the simulation Scenario A1 is considered. The simulation setup of Scenario A2 is used to examine the SLAT accuracy with different reference cluster sizes, while Scenario A3 is employed to evaluate the SLAT performance with different sensor location precisions. In addition, Scenario A4 is simulated to show the degree of fluctuation in the approximation of the balance state $\boldsymbol{J}_{\mathrm{BE}}^{\mathbf{x} \star}$ (i.e., Theorem 6). We assume $\gamma=3$, $P_{T}=50, L_{0}=1, d_{0}=1$ and $r_{s}=20[\mathrm{~m}]$ in all simulations.

The parameter settings in TABLE II correspond to a typical localization-aware WSN with shadow fading, where the RSS measurement noise variance (i.e., the inverse of measurement precision expectation $\Omega \psi$ ) is around $18 \mathrm{~dB}$ [46]. Hence, we 
TABLE II

Simulation SETTINGS

\begin{tabular}{ccccccc}
\hline & $\chi$ & $\mathbf{U}_{t}^{i}, \mathbf{U}_{t}^{j}$ & $\Omega$ & $\psi$ & $M_{t}, N_{t}$ & $K$ \\
\hline A1 & $\star$ & $\star$ & $\star$ & $\star$ & 6 & 50 \\
A2 & $1 / 10 \mathbf{I}$ & $1 / 5 \mathbf{I}$ & $1 / 150$ & 8 & $3: 15$ & 50 \\
A3 & $1 / 100 \mathbf{I}$ & $\star$ & $1 / 50$ & 8 & 6 & 50 \\
A4 & $\star$ & $1 / 50$ & $1 / 150$ & 8 & 6 & 50 \\
\hline
\end{tabular}

$\star$ Refer to the corresponding text for detailed settings. $K$ denotes the total number of time instants in simulations.

set $\Omega=1 / 150$ and $\psi=8$ in simulations such that the measurement precision expectation $\Omega \psi=8 / 150$ is comparable to the inverse of noise variance.

All sensor nodes are assumed to be deployed uniformly in the network coverage area, and the mobile target is assumed to randomly move within this area. The reference sensor nodes of the mobile target are assumed to be uniformly distributed inside a circle area around the target, given by $\mathbb{C}_{t}=\left\{\breve{\mathbf{x}}_{t}\right.$ : $\left.\left\|\breve{\mathbf{x}}_{t}-\mathbf{x}_{t}\right\|_{2} \leq r_{s}\right\}$, to remove the influence of the sensor node deployments on tracking performance, so is the setup for location calibration of sensor nodes. One exemplary case of mobile target mobility with transition precision $\chi=0.1 \mathbf{I}$ is shown in Fig. 8.

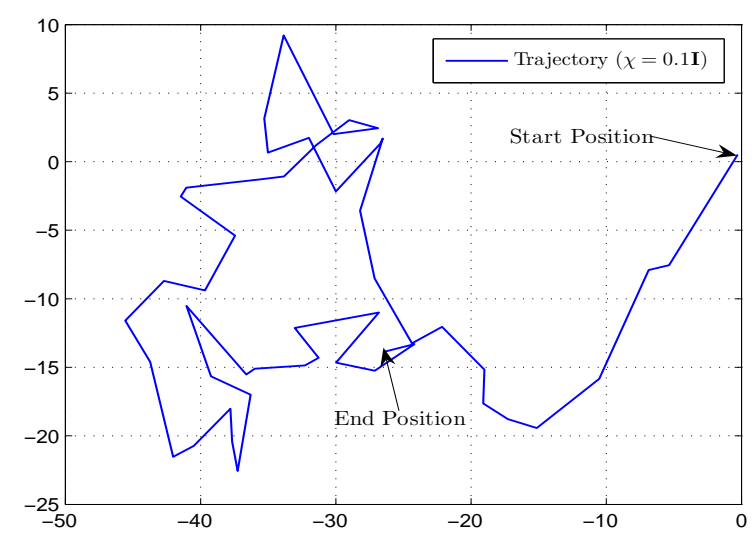

Fig. 8. An exemplary case of mobile target movement.

\section{B. Asymptotic Error Propagation}

1) Impact of Measurement Precision and Target Mobility: The scale parameter $\Omega$ and the associated $\operatorname{DoF} \psi$ jointly indicate the severity of shadow fading. In the meanwhile, the transition precision matrix $\chi$ is inversely related to the randomness of target mobility. In order to assess the impact of spatial-temporal-domain random measurement precision and the target mobility on the SLAT performance, i.e., the tracking EP convergence, balance state of tracking accuracy, Scenario A1 is simulated. The corresponding simulation settings of Scenario $A 1$ are given in TABLE II.

Firstly, the tracking EP convergence properties are shown in Fig. 9, where $\psi=8$ and $\mathbf{U}_{t}^{i}=\mathbf{I}$. We can see that, when the measurement precision expectation $\Omega \psi$ is the same in different cases, the initial tracking errors with different $\chi$

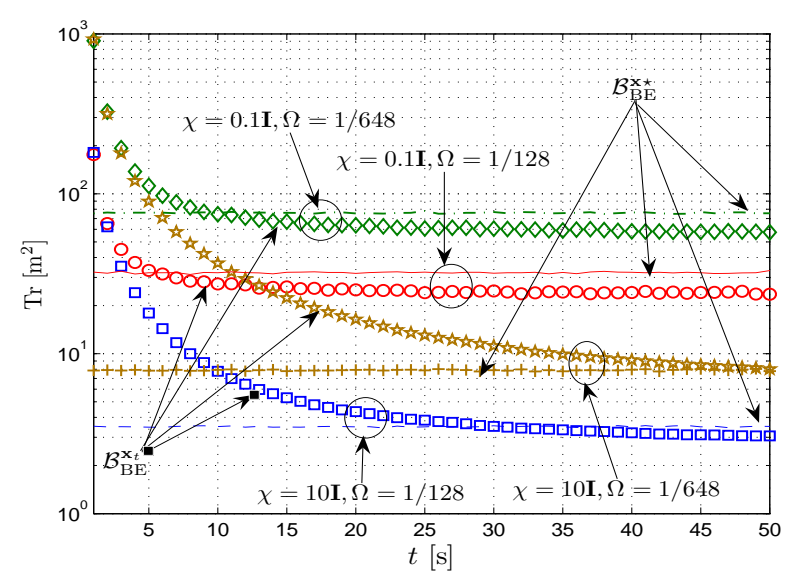

Fig. 9. Tracking EP convergence behavior over different target mobilities and measurement precisions, where $\psi=8$ and $\mathbf{U}_{t}^{i}=\mathbf{I}$.

are also the same (in fact, it is $\boldsymbol{\Psi}_{t}^{\prime \prime}$, see Eq. (98) and Fig. 10 for more details), because when $t=1$ the initial prediction information $\mathcal{I}_{\mathrm{P}}^{\mathrm{x}_{1}}=\mathbf{0}$ since there is no priori information of the initial target location (i.e., $\boldsymbol{J}_{\mathrm{BE}}^{\mathbf{x}_{0}}=\mathbf{0}$ ). In addition, the target tracking error $\mathcal{B}_{\mathrm{BE}}^{\mathbf{x}_{t}}$ can approach its balance state $\mathcal{B}_{\mathrm{BE}}^{\mathrm{x} \star}$, although there is still a little gap between $\mathcal{B}_{\mathrm{BE}}^{\mathbf{x}_{t}}$ and $\mathcal{B}_{\mathrm{BE}}^{\mathrm{x} \star}$, due to the non-symmetric distribution of random $\mathcal{H}_{t}$. Moreover, a larger measurement precision expectation $\Omega \psi$ leads to a faster tracking EP convergence and a smaller balance error.

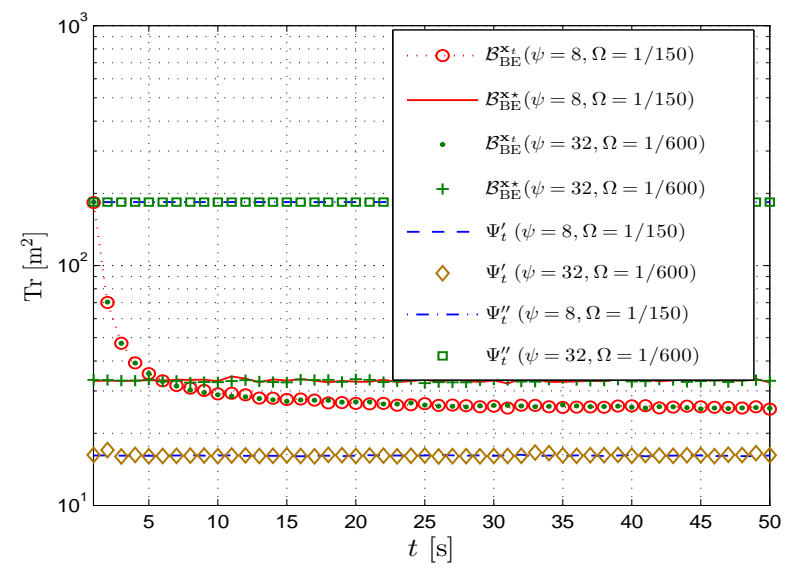

Fig. 10. Tracking EP convergence behavior with a fixed measurement precision expectation. In addition, $\boldsymbol{\chi}=1 / 10 \mathbf{I}, \mathbf{U}_{t}^{i}=\mathbf{I}, \Omega \psi=8 / 150$.

Secondly, we evaluate the tracking EP convergence property in different shadow fading scenarios (with different scale and DoF but with the same expectation of measurement precision). In the simulation, we set $\chi=1 / 10 \mathbf{I}, \mathbf{U}_{t}^{i}=\mathbf{I}$, and the measurement precision expectation is fixed at $\psi \Omega=8 / 150$. As analysed previously, in the environment with spatial-temporaldomain random measurement precision, the tracking EP only depends on the associated expectation, which concurs with the results shown in Fig. 10, where two EP convergence curves (with different $\Omega$ and $\psi$ but the same $\psi \Omega$ ) almost coincide.

In addition, we can see from Fig. 10 that both $\mathcal{B}_{\mathrm{BE}}^{\mathbf{x}_{t}}$ and $\mathcal{B}_{\mathrm{BE}}^{\mathrm{x} \star}$ are upper and lower bounded by $\boldsymbol{\Psi}_{t}^{\prime}$ and $\boldsymbol{\Psi}_{t}^{\prime \prime}$, respectively, as 


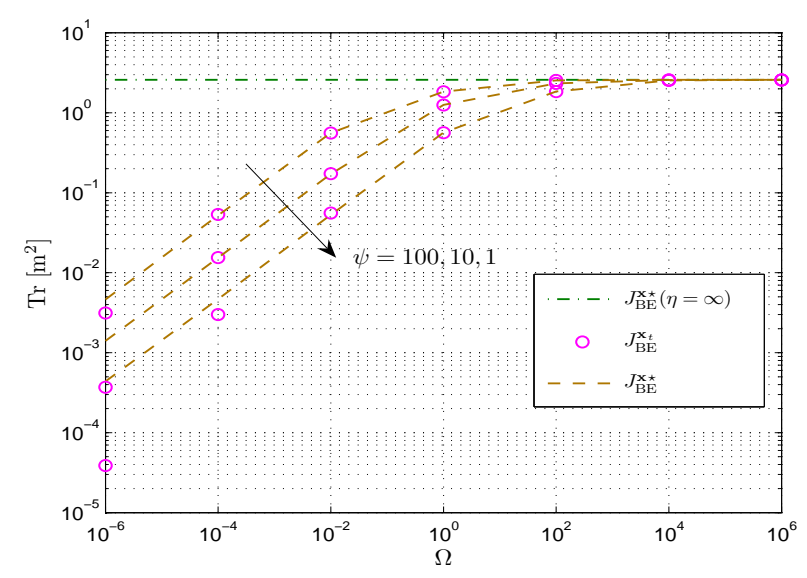

(a) Target tracking accuracy over different levels of shadow fading, where $\chi=0.1 \mathbf{I}$ and $\mathbf{U}_{t}^{i}=1 / 5 \mathbf{I}, \forall i \in \Psi_{t}$.

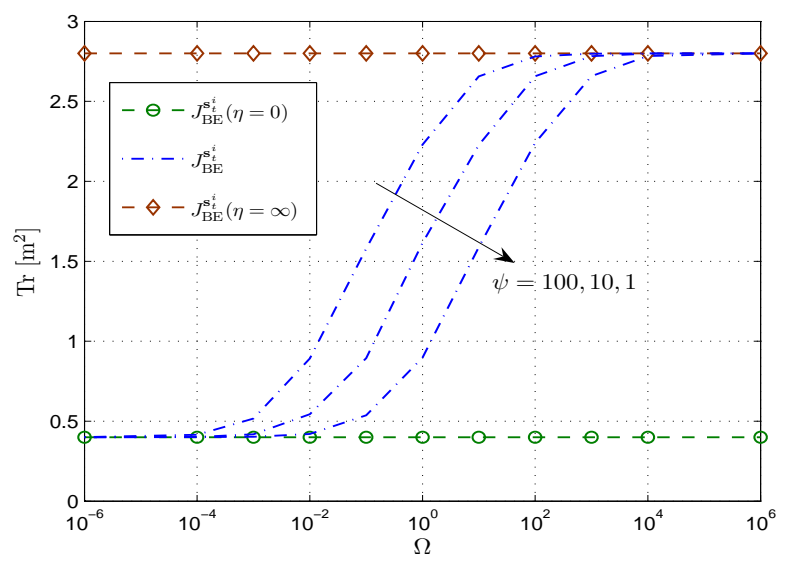

(b) Sensor location calibration accuracy over different levels of shadow fading, where $\mathbf{U}_{t}^{j}=1 / 5 \mathbf{I}, \forall j \in \Phi_{t}^{i}$.

Fig. 11. The SLAT's precision over different levels of shadow fading.

indicated by Eq. (98). The initial tracking error $\mathcal{B}_{\mathrm{BE}}^{\mathrm{x}_{1}}$ equals to $\boldsymbol{\Psi}_{t}^{\prime \prime}$. Moreover, $\boldsymbol{\Psi}_{t}^{\prime}$ and $\boldsymbol{\Psi}_{t}^{\prime \prime}$ are also invariant with a fixed expectation of measurement precision, as shown in Fig. 10.

Thirdly, we assess both tracking and calibration performance over different levels of shadow fading, and we set $\chi=0.1 \mathbf{I}$ and $\mathbf{U}_{t}^{i}, \mathbf{U}_{t}^{j}=1 / 5 \mathbf{I}, \forall i \in \Psi_{t}$ and $\forall j \in \Phi_{t}^{i}$. As shown in Fig. 11(a), both the tracking accuracy $\boldsymbol{J}_{\mathrm{BE}}^{\mathbf{x}_{t}}$ and its balance accuracy $\boldsymbol{J}_{\mathrm{BE}}^{\mathrm{\times}}$ increase with the increase in the measurement precision expectation $\psi \Omega$ and upper bounded by the limit with $\eta=\infty$ (see Eq. (124)). We can also see a similar behaviour of the calibration accuracy $\boldsymbol{J}_{\mathrm{BE}}^{\mathbf{s}_{t}^{i}}$, which is upper and lower bounded by the limits with $\eta=\infty$ and $\eta=0$ (see Eqs. (106) and (107)), respectively, as shown in Fig. 11(b).

Finally, we present the calibration accuracy in different environments with an invariant measurement precision expectation, given by TABLE III. The reference node location precision matrices $\mathbf{U}_{t}^{j}\left(\forall j \in \Phi_{t}^{i}\right)$ of the objective node $\mathbf{s}_{t}^{i}$ are set to be $1 / 5$ I. It is disclosed that the calibration accuracy is also invariant for a fixed expectation of measurement precision.

2) Impact of Reference Cluster Size: To evaluate the impact of the number of reference nodes on both the tracking and calibration accuracies, we simulate Scenario A2 where the reference cluster size is assumed to range from $M_{t}=3$ to 15 (while keeping the sensing range $r_{s}=20$ ). The simulation settings are summarized in TABLE II and the results are shown in Fig. 12. As shown in the figure, both the target tracking accuracy and the node location calibration accuracy scale linearly with the reference cluster size, as revealed by Theorem 9 (see Eqs. (119) and (120)).

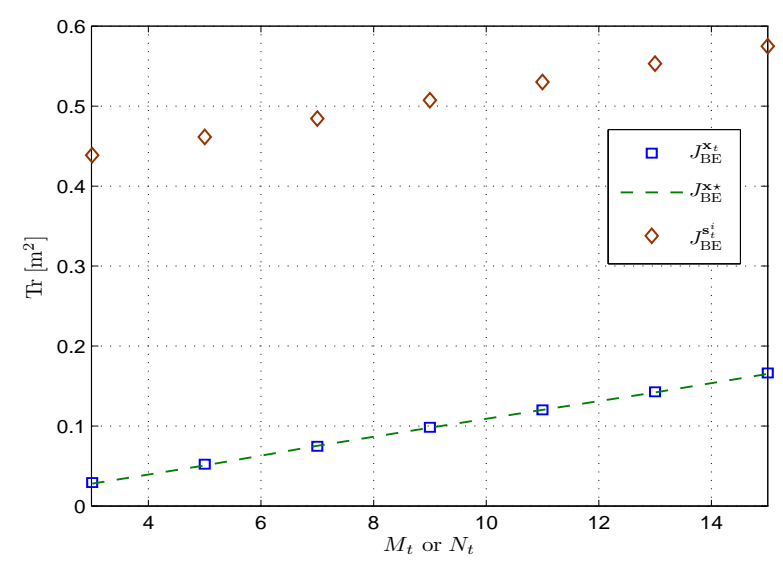

Fig. 12. Tracking and calibration accuracies v.s. reference cluster size.

3) Impact of Reference Node Uncertainties: Scenario A3 is simulated to analyse the tracking and calibration error in different environments with different sensor node location uncertainties.

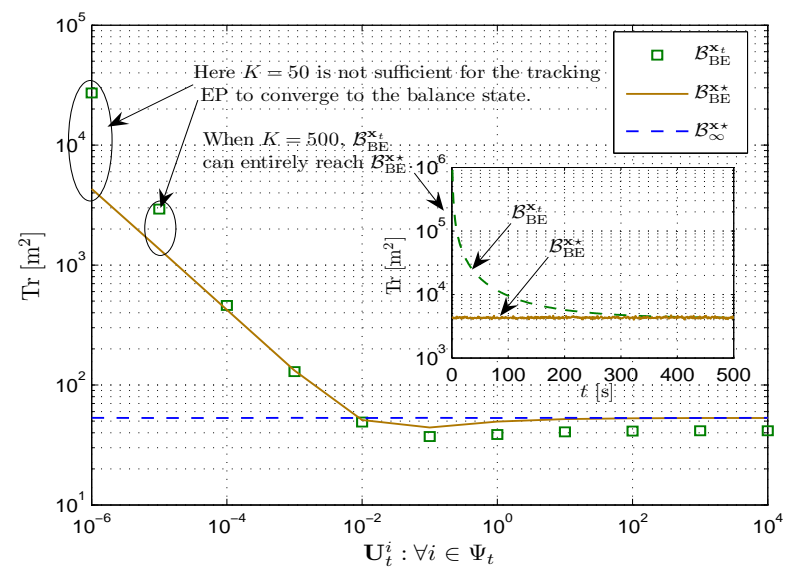

Fig. 13. Target tracking performance v.s. reference sensor node precision.

As shown in Fig. 13, the target tracking error $\mathcal{B}_{\mathrm{BE}}^{\mathrm{x}_{t}}$ and its balance state $\mathcal{B}_{\mathrm{BE}}^{\mathrm{x} \star}$ are reduced with the increase in reference node location precisions $\mathbf{U}_{t}^{i}, \forall i \in \Psi_{t}$. In addition, both $\mathcal{B}_{\mathrm{BE}}^{\mathbf{x}_{t}}$ and $\mathcal{B}_{\mathrm{BE}}^{\mathrm{x} \star}$ asymptotically approach $\mathcal{B}_{\infty}^{\mathbf{x} \star}$ when $\mathbf{U}_{t}^{i} \rightarrow \infty, \forall i \in$ $\Psi_{t}$, as indicated by Eq. (123). We can see from Fig. 13 that, $\mathcal{B}_{\mathrm{BE}}^{\mathbf{x}_{t}}, \mathcal{B}_{\mathrm{BE}}^{\mathbf{x}} \rightarrow \infty$ as $\mathbf{U}_{t}^{i} \rightarrow \mathbf{0}, \forall i \in \Psi_{t}, \forall t=1: K$, which complies with Eq. (112). Similarly, the calibration error $\mathcal{B}_{\mathrm{BE}}^{\mathrm{s}_{t}^{i}}$ is also reduced with the increase in $\mathbf{U}_{t}^{i}$, and asymptotically approaches $\mathcal{B}_{\infty}^{\mathbf{s}_{t}^{i}}$ as $\mathbf{U}_{t}^{i} \rightarrow \infty$, as shown in Fig. 14. Moreover, it is obvious to see from Fig. 14 that, $\mathcal{B}_{\mathrm{BE}}^{\mathbf{s}_{t}^{i}}$ approaches $\mathcal{B}_{\mathbf{0}}^{\mathbf{s}_{t}^{i}}$ as $\mathbf{U}_{t}^{i} \rightarrow \mathbf{0}$, which complies with the conclusion in Eq. (116). 
TABLE III

Calibration Accuracy with Fixed Measurement Precision Expectation

\begin{tabular}{c|ccccc}
\hline$(\psi, \Omega)$ & $(8,1 / 150)$ & $(16,1 / 300)$ & $(24,1 / 450)$ & $(32,1 / 600)$ & $(40,1 / 750)$ \\
\hline $\operatorname{tr}\left(\boldsymbol{J}_{\mathrm{BE}}^{\mathbf{s}}\right)$ & 0.0662 & 0.0690 & 0.0644 & 0.0660 & 0.0669 \\
\hline
\end{tabular}

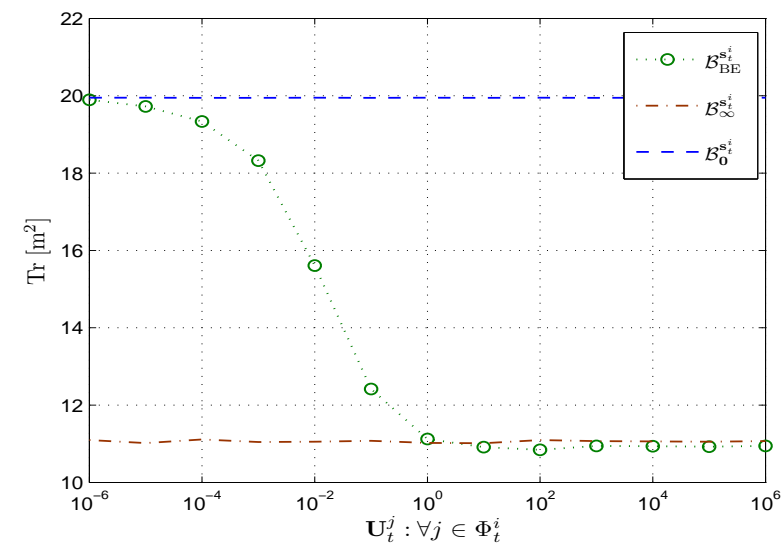

Fig. 14. Sensor location calibration performance over different reference node precisions. The objective node location precision is set to be $\mathbf{U}_{t}^{i}=1 / 10 \mathbf{I}$.

\section{Fluctuation Degree}

Recalling Theorem 6, the fluctuation degree $\mathbf{H}_{t}$ can be approximated by Eq. (102). To examine the approximation accuracy with different degree of target mobility precision $(\chi)$, Scenario A4 is considered in this simulation, the settings of which are given in TABLE II.

Fig. 15 presents the fluctuation degree and its approximation with different transition precision $\chi$. As shown in the figure, the fluctuation degree $\mathbf{H}_{t}$ increases with the increase in transition precision $\chi$. Meanwhile, the fluctuation degree approximation $\widehat{\mathbf{H}}_{t}$ approaches $\mathbf{H}_{t}$ and its normalized approximation error is presented in the subfigure of Fig. 15.

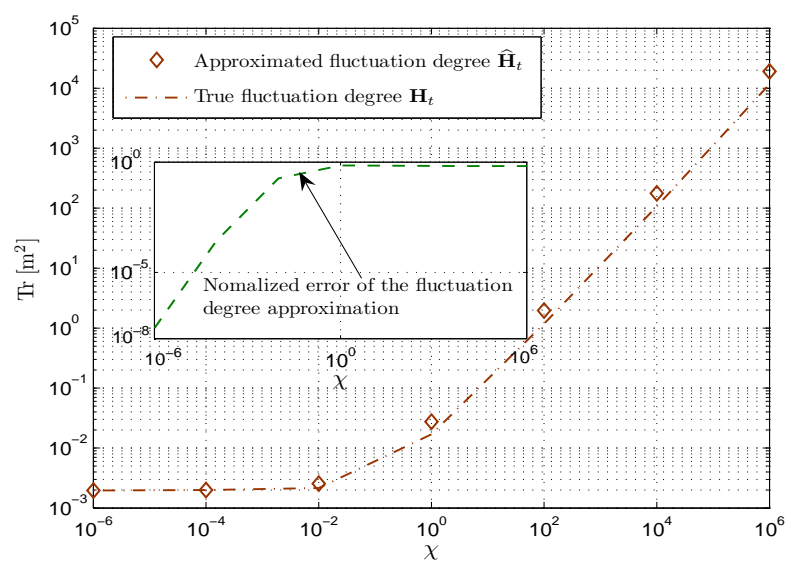

Fig. 15. Fluctuation degree of the balance state $\boldsymbol{J}_{\mathrm{BE}}^{\mathrm{x} \star}$ with different transition precisions. Assume $\boldsymbol{\chi}=\chi \mathbf{I}, \Omega$ is set to be $1 / 150, \psi=8$ and $\mathbf{U}_{t}^{i}=1 / 5 \mathbf{I}$.

The main results for different simulation scenarios are summarized in TABLE IV.

\section{CONCLusions}

In this paper, the error propagation is investigated for both mobile target tracking and sensor node location calibration of the SLAT scheme in WSNs.

The spatial-temporal-domain random measurements owing to different levels of shadow fading, device orientation, strengths of thermal noises, surrounding backgrounds, target mobility and dynamic environments (such as the shopping mall crowded with moving people) have been taken into consideration in the EP analysis to reveal their impact on the SLAT performance.

The mobile target tracking EP and the reference node location calibration EP principles are presented to gain insights into the inherent philosophies of temporal-spatial-domain localization cooperation in the SLAT scheme for WSNs. The presented EP analysis framework can also provide an intuitive way to capture all dominate factors for both linear Gaussian filtering and nonlinear non-Gaussian filtering problem. In particular, a physical interpretation of the EP principles, analogy to the Ohm's Law in electronic circuit theory, is presented to provide an intuitive insight into the EP behaviours.

Furthermore, the EP convergence conditions are derived to guarantee both target tracking error and calibration error converge over time. In addition, the EP properties (e.g., balance state, convergence rate and fluctuation degree of balance state) are evaluated to shed lights on the inherent mechanism of the SLAT scheme. Moreover, the asymptotic performance limits over dependent factors, such as shadow fading, target mobility, reference sensor node density and sensor node location errors are also revealed. Finally, we draw conclusions as follows.

- The EP principles of mobile tracking (which is equivalent to the nonlinear and non-Gaussian filtering with reference variable uncertainties) is identical to that of the classical linear and Gaussian filtering problem. Their EP principles comply with the Ohm's Law in electronic circuits.

- If the overall equivalent measurement information $\mathcal{H}_{t}$ from all reference sensor nodes can compensate the information loss $\mathcal{L}_{t}$ (due to the random target mobility) in the location prediction stage, i.e., $\mathcal{H}_{t} \succ \mathcal{L}_{t}$, the mobile target tracking error $\mathcal{B}_{\mathrm{BE}}^{\mathbf{x}_{t}}$ converges over time.

- The mobile target with higher accuracy at the latest tracking instant will lose more localization information at the location prediction stage, due to its random transition.

- Once the overall equivalent measurement information $\mathcal{H}_{t}$ equals to the information loss $\mathcal{L}_{t}$, the tracking error will converge to the balance state $\mathcal{B}_{\mathrm{BE}}^{\mathrm{x} \star}$, which is give by $\mathcal{B}_{\mathrm{BE}}^{\mathrm{x} \star}=\frac{1}{2} \boldsymbol{\chi}^{-\frac{1}{2}}\left(\mathbf{I}+4 \boldsymbol{\chi}^{\frac{1}{2}} \mathcal{H}_{t}^{-1} \boldsymbol{\chi}^{\frac{1}{2}}\right)^{\frac{1}{2}} \boldsymbol{\chi}^{-\frac{1}{2}}-\frac{1}{2} \boldsymbol{\chi}^{-1}$.

- The mobile tracking accuracy scales linearly with the reference sensor node density. Hence, if the reference cluster size tends to infinity, the final tracking error approaches zero, even all reference sensor locations are 
TABLE IV

Main ReSUlts in VARIOUS Simulation SCENARIOS

\begin{tabular}{|c|c|c|}
\hline Scenario & Results & Concluding remarks \\
\hline \multirow[t]{5}{*}{ A1 } & Fig. 9 & $\begin{array}{l}\text { If the measurement precision expectations are the same, the initial tracking errors are also the same. } \\
\text { A larger measurement precision expectation leads to faster tracking EP convergence and lower balance error. }\end{array}$ \\
\hline & Fig. 10 & $\begin{array}{l}\text { For the spatial-temporal-domain random measurement precision, the tracking error depends on its expectation. } \\
\text { Theorem } 5 \text { associated with the bounds of tracking error balance state is corroborated. }\end{array}$ \\
\hline & Fig. 11(a) & Theorem 11 on the asymptotic limits of balance state $\mathcal{B}_{\mathrm{BE}}^{\mathrm{x} \star}$ over measurement precision is corroborated. \\
\hline & Fig. 11(b) & The calibration performance limits over measurement precision in Eqs. (106) and (107) are corroborated. \\
\hline & TABLE III & The calibration accuracy is invariant for the fixed expectation of measurement precisions. \\
\hline A2 & Fig. 12 & Theorem 9 is corroborated, i.e., tracking and calibration accuracies scale linearly with reference cluster size. \\
\hline A3 & Fig. 13 & $\begin{array}{l}\text { The target tracking error and its balance state are reduced with reference node location precisions. } \\
\text { Theorem } \mathbf{7} \text { on the asymptotic limits of tracking error over reference location precision is corroborated. } \\
\text { Theorem } \mathbf{1 0} \text { on the asymptotic limits of balance state over reference location precision is corroborated. } \\
\text { If all reference node locations are completely inaccurate at every time, the target tracking fails. }\end{array}$ \\
\hline & Fig. 14 & Theorem 8 on the calibration performance limit over reference node location error is corroborated. \\
\hline A4 & Fig. 15 & Theorem 6 on the fluctuation degree of tracking accuracy balance state is corroborated. \\
\hline
\end{tabular}

inaccurate.

- Regarding the spatial-temporal-domain randomness of the measurement precision, the tracking and calibration accuracies are only dependent on the expectation of the measurement precisions.

The mobile target tracking and reference node location calibration EP principles revealed in this paper can be applied to the other range- and angle-based SLAT schemes, in addition to the RSS-based one. Their only difference lies in the expression of geometric resolution factor $\mathbf{A}_{t}^{i}$ with respect to different measurement systems, while the associated error propagation principle is identical.

The spatial-temporal correlated measurements will affect the error propagation philosophies of the simultaneous localization and tracking in WSNs, and we leave this for the future investigation. In addition, all the theoretical analysis in this paper can be utilized to provide guidelines for the optimization of SLAT scheme in terms of reference node selection, network deployment, system optimization, algorithm enhancement, and we leave these optimization issues to our future work.

\section{APPENDIX A \\ DERIVATION OF FIM $\mathcal{I}_{\mathrm{MLE}}^{\mathrm{x}_{t}, \mathrm{x}_{t}}$}

Before deriving the BE-based FIM $\mathcal{I}_{\mathrm{BE}}^{\mathrm{x}_{t}, \mathbf{x}_{t}}$, we first give a useful formulation, i.e., the derivative of likelihood function as follows,

$$
\begin{aligned}
& \nabla_{\mathbf{x}_{t}} \ln p\left(\mathbf{z}_{t} \mid \boldsymbol{\alpha}_{t}\right)=-\frac{1}{2} \nabla_{\mathbf{x}_{t}}\left(\sum_{i \in \Psi_{t}} \mathrm{w}_{t}^{i}\left(\mathrm{z}_{t}^{i}-h\left(\mathbf{x}_{t}, \mathbf{s}_{t}^{i}\right)\right)^{2}\right) \\
= & -\sum_{i \in \Psi_{t}}(\mathrm{w}_{t}^{i}\left(\mathrm{z}_{t}^{i}-h\left(\mathbf{x}_{t}, \mathbf{s}_{t}^{i}\right)\right) \cdot \underbrace{\nabla_{\mathbf{x}_{t}}\left(\mathrm{z}_{t}^{i}-h\left(\mathbf{x}_{t}, \mathbf{s}_{t}^{i}\right)\right)}_{\mathbf{g}_{t}^{i}}) \\
= & -\sum_{i \in \Psi_{t}} \mathrm{w}_{t}^{i}\left(\mathrm{z}_{t}^{i}-h\left(\mathbf{x}_{t}, \mathbf{s}_{t}^{i}\right)\right) \mathbf{g}_{t}^{i},
\end{aligned}
$$

where the derivative $\mathbf{g}_{t}^{i}$ can be calculated as

$$
\begin{aligned}
\mathbf{g}_{t}^{i} & =\nabla_{\mathbf{x}_{t}}\left(\mathrm{z}_{t}^{i}-\phi+10 \gamma \log _{10}\left\|\mathbf{s}_{t}^{i}-\mathbf{x}_{t}\right\|_{2}\right) \\
& =10 \gamma \nabla_{\mathbf{x}_{t}} \log _{10}\left\|\mathbf{s}_{t}^{i}-\mathbf{x}_{t}\right\|_{2}=\frac{10 \gamma}{\ln 10} \frac{\left(\mathbf{x}_{t}-\mathbf{s}_{t}^{i}\right)}{\left\|\mathbf{s}_{t}^{i}-\mathbf{x}_{t}\right\|_{2}^{2}}
\end{aligned}
$$

The MLE-based FIM can thus be further derived as ${ }^{9}$

$$
\begin{aligned}
& \mathcal{I}_{\mathrm{MLE}}^{\mathbf{x}_{t}, \mathbf{x}_{t}}=-\sum_{i \in \Psi_{t}} \mathbb{E}_{\mathbf{z}_{t}, \boldsymbol{\alpha}_{t}}\left\{\nabla_{\mathbf{x}_{t}, \mathbf{x}_{t}^{\top}} \ln p\left(\mathrm{z}_{t}^{i} \mid \mathbf{x}_{t}, \mathbf{s}_{t}^{i}, \mathrm{w}_{t}^{i}\right)\right\} \\
= & \sum_{i \in \Psi_{t}} \mathbb{E}_{\mathbf{z}_{t}, \boldsymbol{\alpha}_{t}}\left\{\nabla_{\mathbf{x}_{t}} \ln p\left(\mathrm{z}_{t}^{i} \mid \mathbf{x}_{t}, \mathbf{s}_{t}^{i}, \mathrm{w}_{t}^{i}\right) \nabla_{\mathbf{x}_{t}^{\top}} \ln p\left(\mathrm{z}_{t}^{i} \mid \mathbf{x}_{t}, \mathbf{s}_{t}^{i}, \mathrm{w}_{t}^{i}\right)\right\} \\
= & \sum_{i \in \Psi_{t}} \mathbb{E}_{\boldsymbol{\alpha}_{t}}\{\mathbf{g}_{t}^{i}\left(\mathrm{w}_{t}^{i}\right)^{2} \underbrace{\mathbb{E}_{\mathbf{z}_{t}}\left\{\left(\mathrm{z}_{t}^{i}-h_{t}^{i}\left(\mathbf{x}_{t}, \mathbf{s}_{t}^{i}\right)\right)^{2}\right\}}_{\left(\mathrm{w}_{t}^{i}\right)^{-1}} \mathbf{g}_{t}^{i \top}\} \\
= & \sum_{i \in \Psi_{t}} \mathbb{E}_{\mathbf{x}_{t}, \mathbf{s}_{t}^{i}, \mathrm{w}_{t}^{i}}\left\{\mathbf{g}_{t}^{i} \mathrm{w}_{t}^{i} \mathbf{g}_{t}^{i \top}\right\} \\
= & \sum_{i \in \Psi_{t}} \eta \mathbf{A}_{t}^{i},
\end{aligned}
$$

where we assume the prior distribution independence, namely $p\left(\mathbf{x}_{t}\right) \Perp p\left(\boldsymbol{\alpha}_{t}^{m}\right), \forall \boldsymbol{\alpha}_{t}^{m} \neq \mathbf{x}_{t}$. In addition, $\eta$ and $\mathbf{A}_{t}^{i}$ are defined in Eqs. (34) and (35), respectively.

\section{APPENDIX B \\ DERIVATION OF $\mathcal{I}_{\text {MLE }}^{\mathbf{s}_{t}^{i}, \mathbf{x}_{t}}$ AND $\mathcal{I}_{\mathrm{MLE}}^{\mathrm{w}_{t}^{i}, \mathbf{x}_{t}}$}

Considering the variable $\forall \boldsymbol{\alpha}_{t}^{m} \neq \mathbf{x}_{t}$, each MLE-based FIM $\mathcal{I}_{\mathrm{MLE}}^{\boldsymbol{\alpha}_{t}^{m}, \mathbf{x}_{t}}$ is generally expressed as

$$
\begin{aligned}
& \mathcal{I}_{\mathrm{MLE}}^{\boldsymbol{\alpha}_{t}^{m}, \mathbf{x}_{t}}=-\mathbb{E}_{\mathbf{z}_{t}, \boldsymbol{\alpha}_{t}}\left\{\nabla_{\boldsymbol{\alpha}_{t}^{m}, \mathbf{x}_{t}^{\top}}\left(\sum_{i \in \Psi_{t}} \ln p\left(\mathrm{z}_{t}^{i} \mid \mathbf{x}_{t}, \mathbf{s}_{t}^{i}, \mathrm{w}_{t}^{i}\right)\right)\right\} \\
= & -\mathbb{E}_{\mathbf{z}_{t}, \boldsymbol{\alpha}_{t}}\left\{\left(\nabla_{\mathbf{x}_{t}, \boldsymbol{\alpha}_{t}^{m \top}} \sum_{i \in \Psi_{t}} \ln \mathcal{N}\left(\mathrm{z}_{t}^{i} \mid h\left(\mathbf{x}_{t}, \mathbf{s}_{t}^{i}\right), \mathrm{w}_{t}^{i}\right)\right)^{\top}\right\} \\
= & \mathbb{E}_{\mathbf{z}_{t}, \boldsymbol{\alpha}_{t}}\left\{\left(\nabla_{\boldsymbol{\alpha}_{t}^{m \top}} \sum_{i \in \Psi_{t}} \mathrm{w}_{t}^{i}\left(\mathrm{z}_{t}^{i}-h\left(\mathbf{x}_{t}, \mathbf{s}_{t}^{i}\right)\right) \mathbf{g}_{t}^{i}\right)^{\top}\right\},
\end{aligned}
$$

where the gradient vector $\mathbf{g}_{t}^{i}$ is given by Eq. (127). Note that,

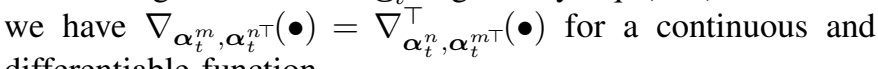
differentiable function.

For the SLAT problem considered in this paper, the FIM $\mathcal{I}_{\mathrm{MLE}}^{\boldsymbol{\alpha}_{t}^{m}, \mathbf{x}_{t}}$ can be further specified as $\mathcal{I}_{\mathrm{MLE}}^{\mathbf{s}_{t}^{i}, \mathbf{x}_{t}}$ and $\mathcal{I}_{\mathrm{MLE}}^{\mathrm{w}_{t}^{i}, \mathbf{x}_{t}}, \forall i \in \Psi_{t}$, which can be deduced as follows.

\footnotetext{
${ }^{9}$ Here we use $\boldsymbol{J}=\mathbb{E}\left\{\nabla_{\boldsymbol{\alpha}_{t}^{n}}(\bullet) \cdot \nabla_{\boldsymbol{\alpha}_{t}^{n \top}}(\bullet)\right\}$ as the FIM formulation.
} 
For $\boldsymbol{\alpha}_{t}^{m}=\mathbf{s}_{t}^{i}, \forall i \in \Psi_{t}$, the FIM $\mathcal{I}_{\text {MLE }}^{\mathbf{s}_{t}^{i}, \mathbf{x}_{t}} \in \mathbb{R}^{D \times D}$ can be derived as

$$
\begin{aligned}
& \boldsymbol{R}_{\mathrm{MLE}}^{\mathbf{s}_{t}^{i}, \mathbf{x}_{t}}=\mathbb{E}_{\mathbf{z}_{t}, \boldsymbol{\alpha}_{t}}\left\{\left(\nabla_{\mathbf{s}_{t}^{i \top}} \sum_{i \in \Psi_{t}} \mathrm{w}_{t}^{i}\left(\mathrm{z}_{t}^{i}-h\left(\mathbf{x}_{t}, \mathbf{s}_{t}^{i}\right)\right) \mathbf{g}_{t}^{i}\right)^{\top}\right\} \\
= & \mathbb{E}_{\mathrm{z}_{t}^{i}, \mathbf{x}_{t}, \mathbf{s}_{t}^{i}, \mathrm{w}_{t}^{i}\left\{\left(\mathrm{w}_{t}^{i} \mathbf{g}_{t}^{i} \mathbf{v}_{t}^{i \top}\right)^{\top}\right\}} \\
& +\underbrace{\mathbb{E}_{\mathbf{z}_{t}^{i}, \boldsymbol{\alpha}_{t}}\left\{\left(\sum_{i \in \Psi_{t}} \mathrm{w}_{t}^{i}\left(\mathrm{z}_{t}^{i}-h\left(\mathbf{x}_{t}, \mathbf{s}_{t}^{i}\right)\right) \cdot \nabla_{\mathbf{s}_{t}^{i \top}} \mathbf{g}_{t}^{i}\right)^{\top}\right\}}_{=0} \\
= & -\eta \mathbf{A}_{t}^{i},
\end{aligned}
$$

where we assume the noise $\epsilon_{t}^{i} \Perp \mathbf{x}_{t}, \mathbf{s}_{t}^{i}, \forall i \in \Psi_{t}$, hence we have $\mathrm{z}_{t}^{i}-h\left(\mathbf{x}_{t}, \mathbf{s}_{t}^{i}\right) \Perp \mathbf{x}_{t}, \mathbf{s}_{t}^{i}$, and the corresponding expectation $\mathbb{E}_{\mathrm{z}_{t}^{i}}\left\{\mathrm{z}_{t}^{i}-h\left(\mathbf{x}_{t}, \mathbf{s}_{t}^{i}\right)\right\}=0$. In addition, the associated gradient $\mathbf{v}_{t}^{i}$ is defined as

$$
\mathbf{v}_{t}^{i}=\nabla_{\mathbf{s}_{t}^{i}}\left(\mathbf{z}_{t}^{i}-h\left(\mathbf{x}_{t}, \mathbf{s}_{t}^{i}\right)\right)=\frac{10 \gamma}{\ln 10} \frac{\mathbf{s}_{t}^{i}-\mathbf{x}_{t}}{\left\|\mathbf{s}_{t}^{i}-\mathbf{x}_{t}\right\|_{2}^{2}} .
$$

The other FIM $\mathcal{I}_{\mathrm{MLE}}^{\mathrm{w}_{t}^{i}, \mathbf{x}_{t}} \in \mathbb{R}^{1 \times D}, \forall i \in \Psi_{t}$, can be derived accordingly as

$$
\begin{aligned}
& \mathcal{I}_{\mathrm{MLE}}^{\mathrm{w}_{t}^{i}, \mathbf{x}_{t}}=\mathbb{E}_{\mathbf{z}_{t}, \boldsymbol{\alpha}_{t}}\left\{\nabla_{\mathrm{w}_{t}^{i}} \sum_{j \in \Phi_{t}^{i}} \mathrm{w}_{t}^{j}\left(\mathrm{z}_{t}^{j}-h\left(\mathbf{x}_{t}, \mathbf{s}_{t}^{j}\right)\right) \mathbf{g}_{t}^{j \top}\right\} \\
= & \underbrace{\mathbb{E}_{\mathrm{z}_{t}^{i}, \mathbf{x}_{t}, \mathbf{s}_{t}^{i}}\left\{\mathrm{z}_{t}^{i}-h\left(\mathbf{x}_{t}, \mathbf{s}_{t}^{i}\right)\right\}}_{=0} \mathbb{E}_{\mathbf{x}_{t}, \mathbf{s}_{t}^{i}}\left\{\mathbf{g}_{t}^{i \top}\right\}=\mathbf{0}_{(1 \times D)} .
\end{aligned}
$$

$$
\begin{gathered}
\text { APPENDIX C } \\
\text { DERIVATION OF } \mathcal{I}_{\mathrm{MLE}}^{\boldsymbol{\alpha}_{t}^{m}, \boldsymbol{\alpha}_{t}^{n}}, \forall \boldsymbol{\alpha}_{t}^{m}, \boldsymbol{\alpha}_{t}^{n} \neq \mathbf{x}_{t}
\end{gathered}
$$

For $\boldsymbol{\alpha}_{t}^{m}=\boldsymbol{\alpha}_{t}^{n}=\mathbf{s}_{t}^{i}$, the MLE-based $\mathcal{I}_{\text {MLE }}^{\mathbf{s}_{t}^{i}, \mathbf{s}_{t}^{i}} \in \mathbb{R}^{D \times D}$ can be reformulated by

$$
\begin{aligned}
& \mathcal{I}_{\mathrm{MLE}}^{\mathbf{s}_{t}^{i}, \mathbf{s}_{t}^{i}}=-\mathbb{E}_{\mathbf{z}_{t}, \boldsymbol{\alpha}_{t}}\left\{\nabla_{\mathbf{s}_{t}^{i}, \mathbf{s}_{t}^{i \top}} \sum_{j \in \Psi_{t}} \ln p\left(\mathrm{z}_{t}^{j} \mid \mathbf{x}_{t}, \mathbf{s}_{t}^{j}, \mathrm{w}_{t}^{j}\right)\right\} \\
= & \mathbb{E}_{\mathbf{z}_{t}, \boldsymbol{\alpha}_{t}}\left\{\nabla_{\mathbf{s}_{t}^{i}} \ln p\left(\mathrm{z}_{t}^{i} \mid \mathbf{x}_{t}, \mathbf{s}_{t}^{i}, \mathrm{w}_{t}^{i}\right) \cdot \nabla_{\mathbf{s}_{t}^{i \top}} \ln p\left(\mathrm{z}_{t}^{i} \mid \mathbf{x}_{t}, \mathbf{s}_{t}^{i}, \mathrm{w}_{t}^{i}\right)\right\} \\
= & \mathbb{E}_{\mathrm{w}_{t}^{i}}\left\{\mathrm{w}_{t}^{i}\right\} \mathbb{E}_{\mathbf{s}_{t}^{i}}\left\{\mathbf{v}_{t}^{i} \mathbf{v}_{t}^{i \top}\right\}=\Omega \psi \mathbf{A}_{t}^{i}=\eta \mathbf{A}_{t}^{i} .
\end{aligned}
$$

The priori FIM $\mathcal{I}_{\mathrm{P}}^{\mathrm{s}_{t}^{i}}$ is given by

$$
\mathcal{I}_{\mathrm{P}}^{\mathbf{s}_{t}^{i}}=-\mathbb{E}_{\mathbf{z}_{t}, \boldsymbol{\alpha}_{t}}\left\{\nabla_{\mathbf{s}_{t}^{i}, \mathbf{s}_{t}^{i \top}} \ln \mathcal{N}\left(\mathbf{s}_{t}^{i} \mid \boldsymbol{\mu}_{t}^{i}, \mathbf{U}_{t}^{i}\right)\right\}=\mathbf{U}_{t}^{i} .
$$

When $\boldsymbol{\alpha}_{t}^{m} \neq \boldsymbol{\alpha}_{t}^{n}$ where $\forall \boldsymbol{\alpha}_{t}^{m}, \boldsymbol{\alpha}_{t}^{n} \neq \mathbf{x}_{t}$, the MLE-based FIMs $\mathcal{I}_{\mathrm{MLE}}^{\boldsymbol{\alpha}_{t}^{m}, \boldsymbol{\alpha}_{t}^{n}}$ can be easily derived as

$$
\begin{aligned}
& \mathcal{I}_{\mathrm{MLE}}^{\mathbf{s}_{t}^{i}, \mathbf{s}_{t}^{j}}=\mathbf{0}_{(D \times D)} \\
& \mathcal{I}_{\mathrm{MLE}}^{\mathbf{s}_{t}^{i}, \mathrm{w}_{t}^{j}}=\mathbf{0}_{(D \times 1)}, \\
& \mathcal{I}_{\mathrm{MLE}}^{\mathrm{w}_{t}^{j}, \mathbf{s}_{t}^{i}}=\mathbf{0}_{(D \times 1)}^{\top}, \\
& \mathcal{I}_{\mathrm{MLE}}^{\mathrm{w}_{t}^{i}, \mathrm{w}_{t}^{j}}=0, \forall i \neq j .
\end{aligned}
$$

\section{APPENDIX D}

DERIVATION OF EQ. (39)

Based on the inverse matrix lemma [76] for invertible matrixes $\mathbf{A}$ and $\mathbf{X}:(\mathbf{A}+\mathbf{X})^{-1}=\mathbf{A}^{-1}-\left(\mathbf{A}^{\top} \mathbf{X}^{-1} \mathbf{A}+\mathbf{A}\right)^{-1}$, we have

$$
\left(\eta \mathbf{A}_{t}^{i}+\mathbf{U}_{t}^{i}\right)^{-1}=\left(\eta \mathbf{A}_{t}^{i}\right)^{-1}-\left(\eta^{2} \mathbf{A}_{t}^{i \top}\left(\mathbf{U}_{t}^{i}\right)^{-1} \mathbf{A}_{t}^{i}+\eta \mathbf{A}_{t}^{i}\right)^{-1} .
$$

Note that, in the SLAT scheme, both $\mathbf{A}_{t}^{i}$ and $\mathbf{U}_{t}^{i}$ are symmetric matrices. Hence, the subtracted information in Eq. (38) is reformulated as

$$
\begin{aligned}
& \sum_{i \in \Psi_{t}} \eta^{2} \mathbf{A}_{t}^{i \top}\left(\eta \mathbf{A}_{t}^{i}+\mathbf{U}_{t}^{i}\right)^{-1} \mathbf{A}_{t}^{i} \\
= & \sum_{i \in \Psi_{t}} \eta \mathbf{A}_{t}^{i \top}-\sum_{i \in \Psi_{t}} \eta \mathbf{A}_{t}^{i \top}\left(\eta \mathbf{A}_{t}^{i \top}\left(\mathbf{U}_{t}^{i}\right)^{-1} \mathbf{A}_{t}^{i}+\mathbf{A}_{t}^{i}\right)^{-1} \mathbf{A}_{t}^{i} \\
= & \sum_{i \in \Psi_{t}} \eta \mathbf{A}_{t}^{i \top}-\sum_{i \in \Psi_{t}}\left(\left(\mathbf{U}_{t}^{i}\right)^{-1}+\left(\eta \mathbf{A}_{t}^{i}\right)^{-1}\right)^{-1}
\end{aligned}
$$

Consequently, the final FIM associated with tracking accuracy can be expressed as Eq. (39).

\section{APPENDIX E}

DERIVATION OF EQ. (72)

Accounting for Eqs. (68) to (70), the linear and Gaussian filtering's final tracking error can be rewritten as

$$
\mathcal{B}_{\mathrm{BE}}^{\mathbf{x}_{t}}=\left(\mathcal{I}_{\mathrm{P}}^{\mathbf{x}_{t}}\right)^{-1}-\mathcal{S}_{t}
$$

where the matrix $\mathcal{S}_{t}$ is formed as

$$
\begin{aligned}
\mathcal{S}_{t} & =\left(\mathcal{I}_{\mathrm{P}}^{\mathbf{x}_{t}}\right)^{-1} \mathcal{Q}_{t}^{\top}\left(\mathcal{Q}_{t}\left(\mathcal{I}_{\mathrm{P}}^{\mathbf{x}_{t}}\right)^{-1} \mathcal{Q}_{t}^{\top}+\mathbf{W}^{-1}\right)^{-1} \mathcal{Q}_{t}\left(\mathcal{I}_{\mathrm{P}}^{\mathbf{x}_{t}}\right)^{-1} \\
& =\left(\mathcal{I}_{\mathrm{P}}^{\mathbf{x}_{t}}\right)^{-1}\left(\left(\mathcal{I}_{\mathrm{P}}^{\mathbf{x}_{t}}\right)^{-1}+\mathcal{Q}_{t}^{-1} \mathbf{W}^{-1}\left(\mathcal{Q}_{t}^{\top}\right)^{-1}\right)^{-1}\left(\mathcal{I}_{\mathrm{P}}^{\mathbf{x}_{t}}\right)^{-1}
\end{aligned}
$$

Resorting to the inverse matrix lemma again, the term in $\mathcal{S}_{t}$ can be derived as

$$
\begin{aligned}
& \left(\left(\mathcal{I}_{\mathrm{P}}^{\mathbf{x}_{t}}\right)^{-1}+\mathcal{Q}_{t}^{-1} \mathbf{W}^{-1}\left(\mathcal{Q}_{t}^{\top}\right)^{-1}\right)^{-1} \\
= & \mathcal{I}_{\mathrm{P}}^{\mathbf{x}_{t}}-\left(\left(\mathcal{I}_{\mathrm{P}}^{\mathbf{x}_{t}}\right)^{-1} \mathcal{Q}_{t}^{\top} \mathbf{W} \mathcal{Q}_{t}\left(\mathcal{I}_{\mathrm{P}}^{\mathbf{x}_{t}}\right)^{-1}+\left(\mathcal{I}_{\mathrm{P}}^{\mathbf{x}_{t}}\right)^{-1}\right)^{-1} \\
= & \mathcal{I}_{\mathrm{P}}^{\mathbf{x}_{t}}-\mathcal{I}_{\mathrm{P}}^{\mathbf{x}_{t}}\left(\mathcal{Q}_{t}^{\top} \mathbf{W} \mathcal{Q}_{t}+\mathcal{I}_{\mathrm{P}}^{\mathbf{x}_{t}}\right)^{-1} \mathcal{I}_{\mathrm{P}}^{\mathbf{x}_{t}} .
\end{aligned}
$$

Substituting Eq. (144) into (143), the matrix $\mathcal{S}_{t}$ can be further derived as

$$
\mathcal{S}_{t}=\left(\mathcal{I}_{\mathrm{P}}^{\mathbf{x}_{t}}\right)^{-1}-\left(\mathcal{Q}_{t}^{\top} \mathbf{W} \mathcal{Q}_{t}+\mathcal{I}_{\mathrm{P}}^{\mathbf{x}_{t}}\right)^{-1} .
$$

Substituting Eq, (145) into (142), the final filtering error $\mathcal{B}_{\mathrm{BE}}^{\mathrm{x}_{t}}$ is obtained as

$$
\mathcal{B}_{\mathrm{BE}}^{\mathbf{x}_{t}}=\left(\mathcal{Q}_{t}^{\top} \mathbf{W} \mathcal{Q}_{t}+\mathcal{I}_{\mathrm{P}}^{\mathbf{x}_{t}}\right)^{-1} .
$$

By taking the inverse of both sides, the filtering accuracy $\boldsymbol{J}_{\mathrm{BE}}^{\mathbf{x}_{t}}$ can be written as Eq. (72).

\section{APPENDIX F \\ Proof of THEOREM 1}

Proof: Based on Definition 1, we know the condition of $\mathcal{H}_{t} \succ \mathcal{L}_{t}$ is equivalent to $\mathcal{H}_{t}+\mathcal{I}_{\mathrm{P}}^{\mathbf{x}_{t}} \succ \boldsymbol{J}_{\mathrm{BE}}^{\mathbf{x}_{t-1}}$. Since $\mathcal{H}_{t}+$ $\mathcal{I}_{\mathrm{P}}^{\mathbf{x}_{t}}=\boldsymbol{J}_{\mathrm{BE}}^{\mathrm{x}_{t}}$, thus we have $\boldsymbol{J}_{\mathrm{BE}}^{\mathrm{x}_{t}} \succ \boldsymbol{J}_{\mathrm{BE}}^{\mathrm{x}_{t-1}}$, which means the target tracking error converges over time.

The convergence condition Eq. (93) is thus obtained. 
APPENDIX G

\section{PROOF OF THEOREM 2}

Proof: The calibration error of the objective sensor node $\mathbf{s}_{t}^{i}$ converges means $\boldsymbol{J}_{\mathrm{BE}}^{\mathbf{s}_{t}^{i}} \succ \mathbf{U}_{t}^{i}$, at current time instant $t$.

Based on Eq. (63), we know that the inequality $\boldsymbol{J}_{\mathrm{BE}}^{\mathbf{s}_{t}^{i}} \succ$ $\mathbf{U}_{t}^{i}$ holds only under the condition that at least one reference sensor node $\mathbf{s}_{t}^{j}$, where $j \in \Phi_{t}^{i}$, provides effective measurement information such that

$$
\left(\left(\eta \mathbf{A}_{t}^{i, j}\right)^{-1}+\left(\mathbf{U}_{t}^{j}\right)^{-1}\right)^{-1} \succ \infty
$$

or the target offers effective measurement information, i.e.,

$$
\left(\left(\eta \mathbf{A}_{t}^{i}\right)^{-1}+\left(\mathcal{I}_{\mathrm{BE}}^{\mathbf{x}_{t}}\right)^{-1}\right)^{-1} \succ \infty .
$$

to the objective sensor node $\mathrm{s}_{t}^{i}$, where $i \in \Psi_{t}$.

In the meanwhile, we know the geometric resolution matrices $\mathbf{A}_{t}^{i} \succ \mathbf{0}$ and $\mathbf{A}_{t}^{i, j} \succ \mathbf{0}, \forall j \in \Phi_{t}^{i}$, always hold (see Eqs. (35) and (61)). Hence, only the conditions of either $\mathbf{U}_{t}^{j} \succ \mathbf{0}$ or $\mathcal{I}_{\mathrm{BE}}^{\mathbf{x}_{t}} \succ \mathbf{0}$ and $\eta>0$ are required.

Consequently, the conditions of calibration error convergence in Theorem 2 are obtained.

\section{APPENDIX H Proof OF THEOREM 3}

Proof: Eqs. (94) and (95) can be directly derived based on Eq. (92). We aim to prove the left part of Theorem 3.

We know that when the latest tracking accuracy $\boldsymbol{J}_{\mathrm{BE}}^{\mathbf{x}_{t-1}}$ increases, $\mathcal{B}_{\mathrm{BE}}^{\mathbf{x}_{t-1}}$ decreases, then $\left(\mathcal{B}_{\mathrm{BE}}^{\mathbf{x}_{t-1}}\right)^{\top} \chi \mathcal{B}_{\mathrm{BE}}^{\mathbf{x}_{t-1}}+\mathcal{B}_{\mathrm{BE}}^{\mathbf{x}_{t-1}}$ reduces. Furthermore, according to Eq. (92), the information loss $\mathcal{L}_{t}$ is monotonously decreasing with the term $\left(\mathcal{B}_{\mathrm{BE}}^{\mathbf{x}_{t-1}}\right)^{\top} \chi \mathcal{B}_{\mathrm{BE}}^{\mathbf{x}_{t-1}}+\mathcal{B}_{\mathrm{BE}}^{\mathbf{x}_{t-1}}$. Hence, $\mathcal{L}_{t}$ increases with $\boldsymbol{J}_{\mathrm{BE}}^{\mathbf{x}_{t-1}}$.

Consequently, Theorem 3 is proved.

\section{APPENDIX I}

\section{ProOF OF THEOREM 4}

We know $\mathcal{H}_{t}, \mathcal{B}_{\mathrm{BE}}^{\mathbf{x}_{t-1}}$ and the transition precision matrix $\chi$ are positive semidefinite, thus is also symmetric. $\mathcal{B}_{\mathrm{BE}}^{\mathrm{x}_{t-1}^{\top}} \chi \mathcal{B}_{\mathrm{BE}}^{\mathbf{x}_{t-1}}$ can therefore be formulated as

$$
\begin{aligned}
& \mathcal{B}_{\mathrm{BE}}^{\mathbf{x}_{t-1}^{\top}} \chi \mathcal{B}_{\mathrm{BE}}^{\mathbf{x}_{t-1}} \\
& =\left(-\frac{1}{2} \chi^{-1}+\frac{1}{2} \chi^{-\frac{1}{2}}\left(\mathbf{I}+4 \chi^{\frac{1}{2}} \mathcal{H}_{t}^{-1} \chi^{\frac{1}{2}}\right)^{\frac{1}{2}} \chi^{-\frac{1}{2}}\right)^{\top} \chi \\
& \cdot\left(-\frac{1}{2} \chi^{-1}+\frac{1}{2} \chi^{-\frac{1}{2}}\left(\mathbf{I}+4 \chi^{\frac{1}{2}} \mathcal{H}_{t}^{-1} \chi^{\frac{1}{2}}\right)^{\frac{1}{2}} \chi^{-\frac{1}{2}}\right) \\
& =\frac{1}{4} \chi^{-1}-\frac{1}{2} \chi^{-\frac{1}{2}}\left(\mathbf{I}+4 \chi^{\frac{1}{2}} \mathcal{H}_{t}^{-1} \chi^{\frac{1}{2}}\right)^{\frac{1}{2}} \chi^{-\frac{1}{2}} \\
& +\frac{1}{4} \chi^{-\frac{1}{2}}\left(\mathbf{I}+4 \chi^{\frac{1}{2}} \mathcal{H}_{t}^{-1} \chi^{\frac{1}{2}}\right) \chi^{-\frac{1}{2}} \\
& =\frac{1}{2} \chi^{-1}-\frac{1}{2} \boldsymbol{\chi}^{-\frac{1}{2}}\left(\mathbf{I}+4 \boldsymbol{\chi}^{\frac{1}{2}} \mathcal{H}_{t}^{-1} \boldsymbol{\chi}^{\frac{1}{2}}\right)^{\frac{1}{2}} \boldsymbol{\chi}^{-\frac{1}{2}}+\mathcal{H}_{t}^{-1} \\
& =-\mathcal{B}_{\mathrm{BE}}^{\mathbf{x}_{t-1}}+\mathcal{H}_{t}^{-1} \text {. }
\end{aligned}
$$

Equivalently, we have

$$
\mathcal{B}_{\mathrm{BE}}^{\mathbf{x}_{t-1} \top} \chi \mathcal{B}_{\mathrm{BE}}^{\mathbf{x}_{t-1}}+\mathcal{B}_{\mathrm{BE}}^{\mathbf{x}_{t-1}}=\mathcal{H}_{t}^{-1} .
$$

This proves Theorem 4 that Eq. (97) is the solution of the balance function (96).

\section{APPENDIX J}

\section{PROOF OF THEOREM 5}

Proof: Based on the tracking EP equation in Eq. (39), the target tracking accuracy asymptotically follows

$$
\begin{gathered}
\lim _{\boldsymbol{J}_{\mathrm{BE}}^{\mathbf{x}_{t-1} \rightarrow \mathbf{0}}} \boldsymbol{J}_{\mathrm{BE}}^{\mathbf{x}_{t}}=\mathcal{H}_{t}, \\
\lim _{\boldsymbol{J}_{\mathrm{BE}}^{\mathbf{x}_{t-1} \rightarrow \infty}} \boldsymbol{J}_{\mathrm{BE}}^{\mathbf{x}_{t}}=\mathcal{H}_{t}+\boldsymbol{\chi} .
\end{gathered}
$$

Namely, the tracking accuracy $\boldsymbol{J}_{\mathrm{BE}}^{\mathbf{x}_{t}}$ ranges from $\mathcal{H}_{t}$ to $\mathcal{H}_{t}+$ $\chi$. We also know that the present tracking accuracy $\boldsymbol{J}_{\mathrm{BE}}^{\mathbf{x}_{t}}$ is nondecreasing with increase in its previous tracking accuracy $\boldsymbol{J}_{\mathrm{BE}}^{\mathbf{x}_{t-1}}$, therefore $\mathcal{H}_{t} \preceq \boldsymbol{J}_{\mathrm{BE}}^{\mathbf{x}_{t}} \preceq \mathcal{H}_{t}+\boldsymbol{\chi}$. Hence the balance state $\mathcal{B}_{\mathrm{BE}}^{\mathrm{x} \star}$ of tracking error is bounded by Eq. (98).

\section{APPENDIX K \\ Proof OF THEOREM 6}

Proof: The balance accuracy $\boldsymbol{J}_{\mathrm{BE}}^{\mathrm{x} \star}$ is the solution to the balance function in Eq. (96), therefore

$$
\left(\left(\boldsymbol{J}_{\mathrm{BE}}^{\mathrm{x} \star}\right)^{-1} \boldsymbol{\chi}\left(\boldsymbol{J}_{\mathrm{BE}}^{\mathrm{x} \star}\right)^{-1}+\left(\boldsymbol{J}_{\mathrm{BE}}^{\mathrm{x} \star}\right)^{-1}\right)^{-1}=\mathcal{H}_{t} .
$$

Let's define a linearization matrix

$$
\mathbf{G}_{t}=\frac{\mathrm{d} \operatorname{tr}\left(\left(\left(\boldsymbol{J}_{\mathrm{BE}}^{\mathbf{x} \star \top}\right)^{-1} \chi\left(\boldsymbol{J}_{\mathrm{BE}}^{\mathbf{x} \star}\right)^{-1}+\left(\boldsymbol{J}_{\mathrm{BE}}^{\mathbf{x} \star}\right)^{-1}\right)^{-1}\right)}{\mathrm{d} \boldsymbol{J}_{\mathrm{BE}}^{\mathbf{\times} \star}}
$$

where $\operatorname{tr}(\bullet)$ denotes the matrix trace.

Based on the above definition, $\mathbf{G}_{t}$ can be further derived as

$$
\begin{aligned}
& \mathbf{G}_{t}=\frac{\mathrm{d} \operatorname{tr}\left(\left(\left(\boldsymbol{J}_{\mathrm{BE}}^{\mathbf{x} \star \top}\right)^{-1} \chi\left(\boldsymbol{J}_{\mathrm{BE}}^{\mathbf{x} \star}\right)^{-1}+\left(\boldsymbol{J}_{\mathrm{BE}}^{\mathbf{x} \star}\right)^{-1}\right)^{-1}\right)}{\mathrm{d} \boldsymbol{J}_{\mathrm{BE}}^{\mathbf{x} \star}} \\
& =\left(\left(\boldsymbol{J}_{\mathrm{BE}}^{\mathbf{\times} \star}\right)^{-1}\left(\left(\boldsymbol{J}_{\mathrm{BE}}^{\mathbf{x} \star}\right)^{-1} \chi^{\top}\left(\boldsymbol{J}_{\mathrm{BE}}^{\mathbf{x} \star}\right)^{-1}+\left(\boldsymbol{J}_{\mathrm{BE}}^{\mathbf{x} \star}\right)^{-1}\right)^{-1}\right. \\
& \cdot\left(\chi^{\top}\left(\boldsymbol{J}_{\mathrm{BE}}^{\mathrm{x} \star}\right)^{-1}+\left(\boldsymbol{J}_{\mathrm{BE}}^{\mathrm{x} \star \top}\right)^{-1} \chi+\mathbf{I}\right) \\
& \left.\cdot\left(\left(\boldsymbol{J}_{\mathrm{BE}}^{\mathbf{x} \star}\right)^{-1} \chi^{\top}\left(\boldsymbol{J}_{\mathrm{BE}}^{\mathbf{x} \star}\right)^{-1}+\left(\boldsymbol{J}_{\mathrm{BE}}^{\mathbf{x} \star}\right)^{-1}\right)^{-1}\left(\boldsymbol{J}_{\mathrm{BE}}^{\mathbf{x} \star}\right)^{-1}\right)^{\top} \\
& =\mathcal{B}_{\mathrm{BE}}^{\mathrm{x} \star \top}\left(\mathcal{B}_{\mathrm{BE}}^{\mathrm{x} \star \top} \chi^{\top} \mathcal{B}_{\mathrm{BE}}^{\mathrm{x} \star}+\mathcal{B}_{\mathrm{BE}}^{\mathrm{x} \star \top}\right)^{-1}\left(\chi^{\top} \mathcal{B}_{\mathrm{BE}}^{\mathrm{x} \star}+\mathcal{B}_{\mathrm{BE}}^{\mathrm{x} \star} \boldsymbol{\chi}+\mathbf{I}\right) \\
& \cdot\left(\mathcal{B}_{\mathrm{BE}}^{\mathrm{x} \star \top} \chi^{\top} \mathcal{B}_{\mathrm{BE}}^{\mathrm{x} \star}+\mathcal{B}_{\mathrm{BE}}^{\mathrm{x} \star \top}\right)^{-1} \mathcal{B}_{\mathrm{BE}}^{\mathrm{x} \star \top} \\
& =\left(\mathcal{B}_{\mathrm{BE}}^{\mathbf{x} \star \top} \chi+\mathbf{I}\right)^{-1}\left(\chi^{\top} \mathcal{B}_{\mathrm{BE}}^{\mathrm{x} \star}+\mathcal{B}_{\mathrm{BE}}^{\mathbf{x} \star} \chi+\mathbf{I}\right)\left(\chi^{\top} \mathcal{B}_{\mathrm{BE}}^{\mathbf{x} \star}+\mathbf{I}\right)^{-1} \text {. }
\end{aligned}
$$

Hence, $\mathbf{G}_{t}$ is finally expressed as Eq. (103).

According to the nonlinear and non-Gaussian estimation theory (refer to Eq. (77)) [26], the estimation precision is quadratically scaled with the linearization coefficient. Hence the precision of balance state $\boldsymbol{J}_{\mathrm{BE}}^{\mathrm{x} \star}$ can be approximated by $\left\langle\mathbf{G}_{t}^{\top} \mathbf{V} \mathbf{G}_{t}\right\rangle_{\mathbf{x}_{t}, \mathbf{s}_{t}}$.

Consequently, the fluctuation degree $\mathbf{H}_{t}$ of balance state $\boldsymbol{J}_{\mathrm{BE}}^{\mathbf{x}}$ is approximated by $\widehat{\mathbf{H}}_{t}$, as given in Eq. (102).

\section{APPENDIX L \\ ProOF OF THEOREM 9}

Proof: Consider a general scenario where all nodes are uniformly deployed inside the whole area, i.e., there is no special deployment for any sensor node. Hence, when adding more sensor nodes into the reference cluster, these sensor nodes may appear anywhere (inside the sensing area) with 
a uniform probability: $\boldsymbol{\mu}_{t}^{i} \sim p\left(\boldsymbol{\mu}_{t}^{i} \mid \mathbf{x}_{t}, r_{s}\right), \forall i$, where $\mathbf{x}_{t}$ and $r_{s}$ represents the center and the radius of the area, respectively.

Moreover, the location precisions $\left\{\mathbf{U}_{t}^{i} \mid \forall i \in \Psi_{t}\right\}$ of all reference nodes are assumed to be independently and identically distributed, i.e., $\mathbf{U}_{t}^{i} \sim p\left(\mathbf{U}_{t}^{i} \mid \mathcal{Z}, \wp\right), \forall i \in \Psi_{t}$, where $\mathcal{Z}$ and $\wp$ are the dependent parameters.

Under these two assumptions, the expectation of the geometric-resolution matrix $\mathbf{A}_{t}^{i}$ can be generally expressed as

$$
\begin{aligned}
\mathbb{E}_{\boldsymbol{\mu}_{t}^{i}, \mathbf{U}_{t}^{i}}\left\{\mathbf{A}_{t}^{i}\right\} & =\int p\left(\mathbf{x}_{t}, \mathbf{s}_{t}^{i}, \boldsymbol{\mu}_{t}^{i}, \mathbf{U}_{t}^{i} \mid \mathcal{X}\right) \mathbf{A}_{t}^{i} \mathrm{~d}\left(\mathbf{x}_{t}, \mathbf{s}_{t}^{i}, \boldsymbol{\mu}_{t}^{i}, \mathbf{U}_{t}^{i}\right) \\
& =\boldsymbol{\Lambda}_{t} \mid \mathcal{X},
\end{aligned}
$$

where $\mathcal{X}$ denotes the set of these dependent parameters, i.e., $\mathcal{Z}, \wp$ and $r_{s}$.

Note that, in such a generalized situation, the node locations and precisions $\left\{\boldsymbol{\mu}_{t}^{i}, \mathbf{U}_{t}^{i} \mid \forall i \in \Psi_{t}\right\}$ are no longer deterministic and known, since we only assume there are $M_{t}$ reference nodes around the target, while their locations $\boldsymbol{\mu}_{t}^{i}$ and precisions $\mathbf{U}_{t}^{i}$ are unknown.

As shown in Eq. (156), for difference reference node $\mathbf{s}_{t}^{i}$, the associated expectation $\mathbb{E}_{\boldsymbol{\mu}_{t}^{i}, \mathbf{U}_{t}^{i}}\left\{\mathbf{A}_{t}^{i}\right\}$ depends on the same distribution parameter set $\mathcal{X}$. Hence, $\mathbb{E}_{\boldsymbol{\mu}_{t}^{i}, \mathbf{U}_{t}^{i}}\left\{\mathbf{A}_{t}^{i}\right\}$ of different nodes will approach the same value $\boldsymbol{\Lambda}_{t}$ in such a situation.

In addition, for each node location uncertainty, we have

$$
\mathbb{E}_{\mathbf{U}_{t}^{i}}\left\{\mathbf{U}_{t}^{i}\right\}=\int p\left(\mathbf{U}_{t}^{i} \mid \mathcal{Z}, \wp\right) \mathbf{U}_{t}^{i} \mathrm{~d} \mathbf{U}_{t}^{i}=\overline{\mathbf{U}}_{t} \mid \mathcal{Z}, \wp .
$$

It thus also converges to the same value denoted by $\overline{\mathbf{U}}_{t}$.

Recalling the general FIM in Eq. (24), under the aforementioned assumptions we come up with a new general FIM (the original's expectation) as

$$
\overline{\boldsymbol{J}}_{\mathrm{BE}}^{\boldsymbol{\alpha}_{t}}=\mathbb{E}_{\boldsymbol{\mu}_{t}, \mathbf{U}_{t}}\left\{-\mathbb{E}_{\mathbf{z}_{t}, \boldsymbol{\alpha}_{t}}\left\{\nabla_{\boldsymbol{\alpha}_{t}, \boldsymbol{\alpha}_{t}^{\top}} \ln p\left(\mathbf{z}_{t}, \boldsymbol{\alpha}_{t}\right)\right\}\right\},
$$

where $\boldsymbol{\mu}_{t}=\left\{\boldsymbol{\mu}_{t}^{i} \mid \forall i \in \Psi_{t}\right\}$ and $\mathbf{U}_{t}=\left\{\mathbf{U}_{t}^{i} \mid \forall i \in \Psi_{t}\right\}$.

In such a case, each FIM item in Eqs. (26) to (33) can be re-derived in a similar form by replacing $\mathbf{A}_{t}^{i}$ and $\mathbf{U}_{t}^{i}$ with $\boldsymbol{\Lambda}_{t}$ and $\overline{\mathbf{U}}_{t}$, respectively. By using the Schur's complement, the equivalent FIM expectation $\overline{\boldsymbol{J}}_{\mathrm{BE}}^{\mathbf{x}_{t}}$ still follows the same form as Eq. (39), i.e.,

$$
\overline{\boldsymbol{J}}_{\mathrm{BE}}^{\mathbf{x}_{t}}=\sum_{i \in \Psi_{t}} \underbrace{\left(\left(\eta \boldsymbol{\Lambda}_{t}\right)^{-1}+\overline{\mathbf{U}}_{t}^{-1}\right)^{-1}}_{\overline{\mathcal{H}}_{t}}+\mathcal{I}_{\mathrm{P}}^{\mathbf{x}_{t}},
$$

where $\mathbf{A}_{t}^{i}$ and $\mathbf{U}_{t}^{i}$ are replaced by $\boldsymbol{\Lambda}_{t}$ and $\overline{\mathbf{U}}_{t}$, respectively. Here, $\overline{\mathcal{H}}_{t}$ represents the averaged information provided by each reference sensor node.

In the same way, we may derive that the node location calibration accuracy also scales linearly with its reference cluster size, according to Eq. (63).

Hence, Theorem 9 is proved.

\section{REFERENCES}

[1] S. Motahari, H. Zang, S. Bali, and P. Reuther, "Mobile applications tracking wireless user location." Global Communications Conference (GLOBECOM), 2012 IEEE. IEEE, 2012.

[2] D. Dardari, A. Conti, C. Buratti and R. Verdone, "Mathematical evaluation of environmental monitoring estimation error through energy-efficient wireless sensor networks." IEEE Trans. Mobile Computing, 6.7 (2007): 790-802.
[3] E. Kaasinen, "User needs for location-aware mobile services," Personal and ubiquitous computing 7.1 (2003): 70-79.

[4] H. Li, L. Sun, Haojin Zhu, X. Lu and X. Cheng, "Achieving privacy preservation in WiFi fingerprint-based localization". INFOCOM, 2014 Proceedings IEEE, vol., no., pp.2337-2345, April 27-May 22014

[5] M. Li, H. Zhu, Z. Gao, S. Chen, L. Yu, S. Hu and K. Ren, "All your location are belong to us: breaking mobile social networks for automated user location tracking," MobiHoc 2014: 43-52

[6] Y. Cao, Z. Sun, N. Wang and M. Riaz, "Geographic-Based Spray-andRelay (GSaR): An Efficient Routing Scheme for DTNs." IEEE Trans. on Veh. Techn. 64.4(2015):1548-1564.

[7] Y. Cao, H. Cruickshank and Z. Sun, "A routing framework for Delay Tolerant Networks based on encounter angle," 2011 7th International Wireless Communications and Mobile Computing Conference (IWCMC) 2231 - 2236, 4-8 July 2011.

[8] M. M. Atia, S. Liu, H. Nematallah, T. B. Karamat and A. Noureldin, "Integrated Indoor Navigation System for Ground Vehicles With Automatic 3-D Alignment and Position Initialization", IEEE Trans. Veh. Techn. vol.64, no.4, pp. 1279-1292, June 2012.

[9] Y. Shen, S. Mazuelas, and M. Z. Win, "Network navigation: Theory and interpretation," IEEE J. Selected Areas in Commun. 30.9 (2012): 18231834.

[10] B. Zhou, Q. Chen, P. Xiao, and L. Zhao, "On the Spatial Error Propagation Characteristics of Cooperative Localization in Wireless Networks," to appear, IEEE Trans. Veh. Techn., 2016.

[11] M. Z. Win, A. Conti, S. Mazuelas, Y. Shen, W. M. Gifford, D. Dardari and M. Chiani, "Network localization and navigation via cooperation," IEEE Commun. Magazine, vol.49, no.5, 2011, pp.56-62.

[12] A. Haeberlen, E. Flannery, A. M. Ladd, A. Rudys, D. S. Wallach and L. E. Kavraki, "Practical Robust Localization over Large-Sclae 802.11 Wireless Networks," Proc. ACM MobiCom, 2004.

[13] H. Wymeersch, J. Lien, and M. Z. Win, "Cooperative localization in wireless networks." Proceedings of the IEEE 97.2 (2009): 427-450.

[14] B. Zhou, Q. Chen, T.-J. Li and P. Xiao, "Online Variational Bayesian Filtering for Mobile Target Tracking in Wireless Sensor Networks", Sensors. 2014; 14(11): 21281-21315.

[15] A. Goldsmith, Wireless Communications, Cambridge University Press, UK, 2005.

[16] M. Vemula, M. F. Bugallo and P. M. Djuric, "Sensor Self-localization with Beacon Position Uncertainty," Signal Processing, Vol.89, No.6, 2009, pp.1144-1154.

[17] B. Huang, C. Yu, B. D. O. Anderson, "Analyzing error propagation in range-based mulithop sensor localization." CDC/CCC 2009. Proceedings of the 48th IEEE Conference on, 2009, pp.865 - 870.

[18] P. Tarrio, M. Cesana, M. Tagliasacchi, A. Redondi, L. Borsani and J. R. Casar, "An energy-efficient strategy for combined RSS-PDR indoor localization," 2011 IEEE International Conference on Pervasive Computing and Communications Workshorps (PERCOM Workshops), IEEE, Seattle, WA, 2011, pp.619-624

[19] T. Wang, G. Leus, and L. Huang, "Ranging Energy Optimization for Robust Sensor Positioning Based on Semidefinite Programming," IEEE Trans. Signal Processing, vol.57, no.12, Dec. 2009, pp.4777-4787

[20] Y. Shen, W. Dai, and M. Z. Win, "Power Optimization for Network Localization," IEEE/ACM Trans. Networking, vol.22, no.4, Aug. 2014 pp. $1337-1350$

[21] S. Tomic, M. Beko, R. Dinis, and V. Lipovac, "Efficient Estimator for Distributed RSS-based Localization in Wireless Sensor Networks", 11th International Wireless Communications and Mobile Computing Conference, Dubrovnik, Croatia, August 24-28, 2015.

[22] C. M. Bishop, Pattern recognition and machine learning. Springer, AUG. 2006,

[23] F. Li, R. Fergus and P. Perona. "One-shot learning of object categories," IEEE Trans. on Pattern Analysis and Machine Intelligence, vol.28, no.4, 2006, pp.594-611.

[24] M. Angjelichinoski, D. Denkovski, V. Atanasovski, L. Gavrilovska, "Cramer-Rao Lower Bounds of RSS-Based Localization With Anchor Position Uncertainty," IEEE Trans. Inform. Theory, 61.5 (2015): 2807 2834.

[25] I. Sharp, K. Yu and T. Sathyan, "Positional accuracy measurement and error modeling for mobile tracking," IEEE Trans. Mobile Computing, 11.6 (2012): 1021-1032.

[26] S. M. Kay, Fundamentals of Statistical Signal Processing, Volume 2: Detection theory. Prentice Hall PTR, 1998.

[27] S. Narasimhan, and J. Krolik, "Limits on Source Range Estimation Performance in Uncertain Ocean Channel," J. Acoust. Soc. Amer., vol.97, no.1, 1995 
[28] P. Tichavsky, C. H. Muravchik, and A. Nehorai, "Posterior CramerRao Bounds for Discrete-Time Nonlinear Filtering," IEEE Trans. Signal Processing, vol.46, no.5, pp.1386-1396, 1998

[29] Y. Noam, and H. Messer, "The Hybrid Cramer-Rao Lower Bound and the Generalized Gaussian Linear Estimation Problem," 2008 5th IEEE Sensor Array and Multichannel Signal Processing Workshop, Darmstadt, pp.395-399, 2008

[30] H. Messer, "The Hybrid Cramer-Rao Lower Bound - From Practice to Theory," Fourth IEEE Workshop on Sensor Array and Multichannel Processing, 2006, Waltham, MA, 2006, pp.304-307, 2006

[31] E. W. Brankin, "Locally best unbiased estimates," Ann. Math. Statist. vol.20, pp.477-501, 1949.

[32] J. Tabrikian, and J. L. Krolik, "Brankin Bounds for Source Localization in an Uncertain Ocean Environment," IEEE Trans. Signal Processing, vol.47, no.11, pp.2917-2927, 1999

[33] I. Reuven and H. Messer, "A Barankin-Type Lower Bound on the Estimation Error of a Hybrid Parameter Vector," IEEE Trans. Inform. Theory, vol.43, no.3, pp.1084-1093, 1997.

[34] B. Bobrovsky and M. Zakai, "A Lower Bound on the Estimation Error for Certain Difussion Process,' IEEE Trans. Inform. Theory, vol.22, no.1, pp.42-52, 1976

[35] V. M. Chiriac, A. M. Haimovichi, "Ziv-Zakai Lower Bound on Target Localization Estimation in MIMO Radar Systems," 2010 IEEE Radar Conference, Washington, DC, 2010, pp.678-683.

[36] K. L. Bell, Y. Steinberg, Y. Ephraim, and H. L. Van Trees, "Extended Ziv-Zakai lower bound for vector parameter estimation," IEEE Trans. Information Theory, vol.43, no.2, March 1997, pp.624C637.

[37] E. Weinstein and A. J. Weiss, "A General Class of Lower Bounds in Parameter Estimation," IEEE Trans. Information Theory, vol.34, no.2, 1988, pp.338-342.

[38] D. Dardari, A. Conti, U. Ferner, A. Giorgetti, and M. Z. Win, "Ranging With Ultrawide Bandwidth Signals in Multipath Environments," Proceedings of the IEEE, 97.2(2009):404-426, Feb. 2009

[39] H. Chen, G. Wang, Z. Wang, H.C. So and H. V. Poor, "None-light-ofsight Node Localization based on Semi-Definite Programming in Wireless Sensor Networks," IEEE Trans. Wireless Commun., 11.1(2012):108-116.

[40] Y. Shen and M. Z. Win, "Fundamental limits of wideband localizationPart I: A General Framework.” IEEE Trans. Inform. Theory, vol.56, no.10, 2010, pp.4956-4979.

[41] Y. Shen, H. Wymeersch and M. Z. Win, "Fundamental limits of wideband localization-Part II: Cooperative Networks.” IEEE Trans. Inform. Theory, vol.56, no.10, 2010, pp.4956-4979.

[42] J. Teng, H. Snoussi , C. Richard and R. Zhou, "Distributed Variational Filtering for Simultaneous Sensor Localization and Target Tracking in Wireless Sensor Networks", IEEE Trans. Veh. Techn., vol.61, no.5, 2012 pp.2305-2318.

[43] B. Hao, Z. Li, Y., and Ren W. Yin, "On the Cramer-Rao Bound of Multiple Sources Localization Using RDOAs and GROAs in the Presence of Sensor Location Uncertainties," Proc. IEEE WCNC, 2012.

[44] M. R. Gholami, E. G. Strom, H. Wymeersch and S. Gezici, "Upper bounds on position error of a single location estimate in wireless sensor networks.” EURASIP J. Adv. Signal Processing., 2014.1 (2014):1-14.

[45] S. Mazuelas, Y. Shen, and M. Z. Win, "Information coupling in cooperative localization," IEEE Commun. Letters, 15.7 (2011): 737-739.

[46] F. Gustafsson and F. Gunnarsson, "Mobile positioning using wireless networks: possibilities and fundamental limitations based on available wireless network measurements," IEEE Signal Processing Magazine 22.4 (2005): 41-53.

[47] Y. Shen, W. Dai, and M. Z. Win, "Power optimization for network localization," IEEE/ACM Trans. Networking, vol.22, no.4, AUG 2014, pp. $1337-1350$.

[48] C. Kreucher, "Optimal Sensor Placement for a Constellation of Multistatic Narrowband Pixelated Sensors," IEEE Transactions on System Man \& Cybemetics Part C, vol.42, no.6, NOV 2012, pp.1374-1383.

[49] H. Wang, K. Yao, and D. Estrin, "Information-Theoretic Approaches for Sensor Selection and Placement in Sensor Networks for Target Localization and Tracking," Journal of Commun. and networks, vol.7, no.4, DEC 2005, pp.438-449.

[50] M. Chu, H. Haussecker, and F. Zhao, "Scalable information-driven sensor querying and routing for ad hoc heterogeneous sensor networks," Int. J. High Perform. Comput. Appl., vol. 16, no. 3, pp. 293-313, 2002.

[51] E. Ertin, J. W. Fisher, and L. C. Potter, "Maximum mutual information principle for dynamic sensor query problems," Information Processing in Sensor Network. IPSN, 2003, pp.405-416.

[52] P. Pahalawatta, T. N. Pappas, and A. K. Katsaggelos, "Optimal sensor selection for video-based target tracking in a wireless sensor network," IEEE Int. Conf. Image Process., Singapore, 2004, pp. 3073-3076.
[53] L. Liu, X. Zhang, and H. Ma, "Optimal Node Selection for Target Localization in Wireless Camera Sensor Networks," IEEE Trans. Veh. Techn., vol.59, no.7 SEP 2010, pp.3563-3576.

[54] J. Qian, X. Jin, and Y. Zhang, "Energy-Efficient Node Selection for Acoustic Source Localization in Wireless Sensor Network," 2010 6th Inter. Conf. on Wireless Commun. Networking and Mobile Computing (WiCOM), IEEE, Chengdu, 2010, pp.1-4

[55] Y. Wang, and D. Wang, "Energy-Efficient Node Selection for Target Tracking in Wireless Sensor Networks," Int. Journal of Distributed Sensor Networks, vol.9 no.1, JAN 2013, pp.127-137

[56] S. Hadzic, D. Yang, M. Violas and J. Rodriguez, "Energy-Efficient mobile tracking in heterogeneous networks using node selection," EURASIP Journal on Wireless Communications and Networking, 1(2014):1-10

[57] U. A. Khan, S. Kar and J. M. F. Moura, "Distributed sensor localization in random environments using minimal number of anchor nodes, IEEE Trans. Signal Process., vol. 57, no.5, May 2009, pp. 2000-2016.

[58] U. A. Khan, S. Kar and J. M. F. Moura, "DILAND: An algorithm for distributed sensor localization with noisy distance measurements," IEEE Trans. Signal Process., vol.58, no.3, Mar. 2010, pp.1940-1947

[59] S. Mazuelas, A. Bahillo, R. M. Lorenzo, P. Fernandez, F. A. Lago, E. Garcia, J. Blas, and E. J. Abril, "Robust indoor positioning provided by real-time RSSI values in unmodified WLAN networks," IEEE J. Sel. Topics Signal Process., vol. 3, no. 5, Oct. 2009, pp. 821-831,

[60] K. Pahlavan, X. Li, and J. P. Makela, "Indoor geolocation science and technology," IEEE Commun. Mag., vol.40, no.2, Feb. 2002, pp.112-118

[61] A. Conti, M. Guerra, D. Dardari, N. Decarli. and M. Z. Win, "Network experimentation for cooperative localization," IEEE J. Selected Areas in Commun., 30.2(2012):467-475.

[62] T. Pavani, G. Costa, M. Mazzotti, A. Conti, and D. Dardari, "Experimental results on indoor localization techniques through wireless sensors network," 2006 IEEE 63rd Vehicular Technology Conference, Melbourne, Vic., 2006, pp.663-667

[63] A. Conti, D. Dardari, M. Guerra, L. Mucchi and M. Z. Win, "Experimental characterization of diversity navigation,” IEEE Syst. J., vol.8, no.1, Mar. 2014, pp.115-124,

[64] J. Prieto, S. Mazuelas and M. Z. Win, "Context-Aided Inertial Navigation via Belief Condensation," IEEE Transactions on Signal Processing, Vol.64, No.12, 2016, pp. 3250-3261.

[65] F. Zabini and A. Conti, "Inhomogeneous Poisson Sampling of FiniteEnergy Signals With Uncertainties in $\mathbb{R}^{d}$," IEEE Transactions on Signal Processing, Vol.64, No.18, 2016, pp. 4679-4694.

[66] J. Chen, W. Dai, Y. Shen, V. K. N. Lau and M. Z. Win, "Power Management for Cooperative Localization: A Game Theoretical Approach," IEEE Transactions on Signal Processing, Vol.64, No.24, 2016, pp. 6517-6532.

[67] S. Kumar, S. Gil, D. Katabi and D. Rus, "Accurate indoor localization with zero start-up cost," Proc. of the 20th Annual International Conference on Mobile Computing and Networking, ACM, 2014.

[68] N. Bulusu, J. Heidemann and D. Estrin, "GPS-less low-cost outdoor localization for very small devices," IEEE Personal Commun., Vol.7, No.5, 2000, pp.28-34.

[69] M. Chiani, A. Conti and R. Verdone, "Partial compensation signal-levelbased up-link power control to extend terminal battery duration," IEEE Transactions on Vehicular Technology, Vol.50, No.4, pp. 1125-1131, Jul 2001

[70] P. M. Djuric, M. V. Vemula, M. R. Bugallo and J. Miguez, "Noncooperative Localization of Binary Sensors." Proc. IEEE 13th Workshop on Statistical Signal Processing, 2005.

[71] C. Taylor, A. Rahimi, J. Bachrach, H. Shrobe, and A. Gure, "Simultaneous localization, calibration, and tracking in an ad hoc sensor network." Proceedings of the 5th international conference on Information processing in sensor networks. ACM, 2006: 27-33.

[72] T. Camp, J. Boleng, and V. Davies. "A survey of mobility models for ad hoc network research." Wireless communications and mobile computing 2.5 (2002): 483-502.

[73] H. Snoussi, and C. Richard. "Ensemble learning online filtering in wireless sensor networks." Communication systems, 2006. ICCS 2006. 10th IEEE Singapore International Conference on. IEEE, 2006.

[74] J. Vermaak, N. D. Lawrence, and Patrick Prez. "Variational inference for visual tracking." 2003 IEEE Computer Society Conference on Computer Vision and Pattern Recognition (CVPR). IEEE, 2003.

[75] S. Boyed, and L. Vandenberghe, "Convex Optimization," Cambridge Univ. Press, 2004

[76] K. Kohno, Y. Inouye and M. Kawamoto. "A Matrix Pseudo-Inversion Lemma and Its Application to Block-Based Adaptive Blind Deconvolution for MIMO Systems." Proc. of IEEE ISCAS, 2007:3490-3493. 
[77] B. Zhou and Q. Chen, "On the Particle-assisted Stochastic Search Mechanism in Wireless Cooperative Localization", IEEE Trans. Wireless Commun., vol.15, no.7, pp.4765-4777, July, 2016.

[78] M. S Arulampalam, S. Maskell, N. Gordon, and T. lapp, "A tutorial on particle filters for online nonlinear/non-Gaussian Bayesian tracking." IEEE Trans. Signal Processing, 2002, 50.2: 174-188.

[79] K. Friston, J. Mattout, N. Trujillo-Barreto, J. Ashburner, and W. Pennya, "Variational free energy and the Laplace approximation." NeuroImage, ELSEVIER, vol.34, no.1, 2006, pp.220-234.

[80] M. J. Beal, and Z. Ghahramani, "The variational Bayesian EM algorithm for incomplete data: with application to scoring graphical model structures," Bayesian Statistics., Vol. 7 (2003).

[81] S. Arulampalam, and B. Ristic. "Comparison of the particle filter with range parameterized and modified polar EKFs for angle-only tracking." Proceedings of SPIE, The International Society for Optical Engineering, vol.4048, 2000, pp.288-299.

[82] A. Gelb. Applied optimal estimation. MIT press, 1974

[83] A. J. Laub, "A Schur method for solving algebraic Riccati equations." IEEE Trans. Automatic Control 24.6 (1979): 913-921.

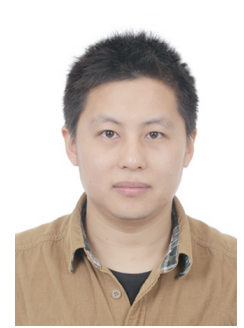

Bingpeng Zhou (S'16-M'17) received the B.Eng degree from Zhongyuan University of Technology, in 2010, and the Ph.D. degree from Southwest Jiaotong University, in 2016, respectively, China. He currently is a Visiting Scholar with the Dept. of Electronic and Computer Engineering, Hong Kong University of Science and Technology, since December, 2016. He was a Visiting Ph.D. Student at the 5G Innovation Centre, University of Surrey, Guildford, U.K., from September to November, 2015. His current research interests include wireless localization \& tracking, Bayesian signal processing and vehicular ad-hoc networks.

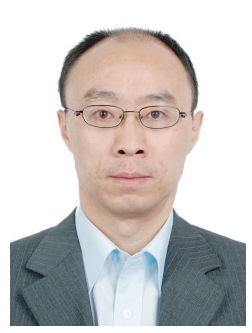

Qingchun Chen (SM'14) received his B.Sc degree and M.Sc degree with honor from Chongqing University, P.R. China, in 1994 and 1997, respectively. He received his Ph.D. degree from Southwest Jiaotong University, P.R. China in 2004. He joined Southwest Jiaotong University since 2004 as an associate professor and then as a full professor since 2009. Dr. Qingchun Chen has authored and coauthored over 100 research papers, two book chapters and 30 patents. Dr. Chen received the 2016 IEEE GLOBECOM Best Paper Award. He served as Associate Editor for IEEE ACCESS (2015-present). His research interest includes wireless communication, wireless network, information coding and signal processing.

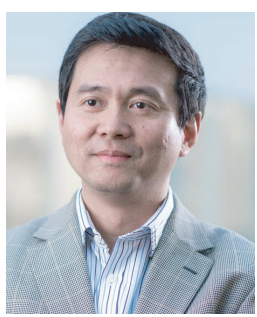

Pei Xiao (SM'11) obtained the B. Eng, MSc and $\mathrm{PhD}$ degree from Huazhong University of Science \& Technology, Tampere University of Technology, Chalmers University of Technology, respectively. He is a Reader at the University of Surrey and is the technical manager of 5G Innovation Centre (5GIC), defining and coordinating research activities, and overseeing major projects in all the work areas within 5GIC (www.surrey.ac.uk/5gic/research). Prior to this, he worked at Newcastle University and Queens University Belfast. He also held positions at Nokia Networks in Finland. He has published extensively in the fields of communication theory and signal processing for wireless communications, and is an inventor in 10 recent 5 GIC patents in the area of $5 \mathrm{G}$ air interface. 